\title{
WestVirginiaUniversity
}

THE RESEARCH REPOSITORY @ WVU

Graduate Theses, Dissertations, and Problem Reports

2012

\section{Electronic structure of delafossites for improved photocatalysis}

\author{
M. Kylee Underwood \\ West Virginia University
}

Follow this and additional works at: https://researchrepository.wvu.edu/etd

\section{Recommended Citation}

Underwood, M. Kylee, "Electronic structure of delafossites for improved photocatalysis" (2012). Graduate Theses, Dissertations, and Problem Reports. 4931.

https://researchrepository.wvu.edu/etd/4931

This Thesis is protected by copyright and/or related rights. It has been brought to you by the The Research Repository @ WVU with permission from the rights-holder(s). You are free to use this Thesis in any way that is permitted by the copyright and related rights legislation that applies to your use. For other uses you must obtain permission from the rights-holder(s) directly, unless additional rights are indicated by a Creative Commons license in the record and/ or on the work itself. This Thesis has been accepted for inclusion in WVU Graduate Theses, Dissertations, and Problem Reports collection by an authorized administrator of The Research Repository @ WVU. For more information, please contact researchrepository@mail.wvu.edu. 


\title{
ELECTRONIC STRUCTURE OF DELAFOSSITES FOR IMPROVED PHOTOCATALYSIS
}

\author{
by \\ M. Kylee Underwood \\ Thesis submitted to the Eberly College of Arts and Sciences \\ at West Virginia University \\ in partial fulfillment of the requirements \\ for the degree of \\ Master of Science \\ in \\ Physics
}

\begin{abstract}
Approved by
James P. Lewis, Ph.D., Committee Chairperson

Alan D. Bristow, Ph.D.

Tudor Stanescu, Ph.D.
\end{abstract}

Department of Physics

Morgantown, West Virginia

2012

Keywords: Electronic band structure, First principle calculation, Tight-binding, Density functional theory, Photovoltaics, Density of states

Copyright 2012 M. Kylee Underwood 


\section{Abstract \\ ELECTRONIC STRUCTURE OF DELAFOSSITES \\ FOR IMPROVED PHOTOCATALYSIS}

\section{by M. Kylee Underwood}

One of the most pressing issues for scientists today is the ever increasing amount of greenhouse gases entering the atmosphere. Carbon dioxide is considered the most prominent greenhouse gas, and emissions from fossil fuel power generation alone represent $26 \%$ of global $\mathrm{CO}_{2}$ emissions. Here we computationally examine the properties of a family of materials called delafossites for the photoreduction of $\mathrm{CO}_{2}$ emissions produced through fossil fuel power generation. These materials show promise to reduce $\mathrm{CO}_{2}$ into usable products such as methane through photoelectrochemical reduction. Delafossites are of interest due to the discrepancy between their fundamental and optically measured band gaps. Due to inversion symmetry a direct transition between the valence and conductions bands is forbidden resulting in an optically measured band gap in the UV region. To narrow the band gap, Huda proposed B-site alloying or doping with the intent to break the inversion symmetry. We expand upon the principle of this proposal, with a focus on computational work. We show the photocatalytic improvement of delafossite material $\mathrm{CuGaO}_{2}$ through B-site doping to obtain $\mathrm{CuGa}_{1-\mathrm{x}} \mathrm{Fe}_{\mathrm{x}} \mathrm{O}_{2}$. 


\section{ACKNOWLEDGMENTS}

First of all, I thank my family for their tremendous support of everything I endeavor to do. You never stop believing in me even when I stop believing in myself. Thanks to my mom, Tisha, my dad, Tim, and my brother, Ben, for picking me up and pushing me forward. Your love and prayers mean the world to me.

I owe many thanks to the guidance of Dr. James P. Lewis. You have taught me just as much about life as you have about science. Thanks for putting up with me for so long and pretending to understand when my plate got full and times got rough. Thanks to Barry Haycock for much technical and moral support inside and outside of the lab. You are a great mentor, and even more so a great friend. I would like to thank all of the people who read my thesis drafts and provided feedback including Cecil O’Dell, and Craig Tenney.

I also give many thanks to Dr. Christopher Matranga and Dr. Jonathan Lekse for their collaboration and thought provoking discussions. I would also like to thank Dr. Alan Bristow and Dr. Tudor Stanescu for their time and patience as members of my committee.

Finally, I would like to thank God for the intelligence and persistence to have had made it this far. Thank You for the beauty that I find in the complexities of this world that I study. Thank You for your patience, love, and mercy at the end of my long days. Without You, I would not be the person I am today, nor would any of this be possible. 


\section{Table of Contents}

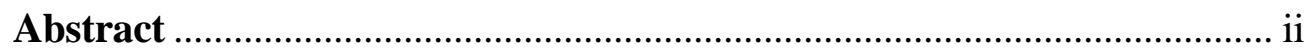

Acknowledgments ............................................................................................. iii

Table of Contents ...........................................................................................

LIST OF FIGURES ……………………………………………………....

LIST OF TABLES …………………………………...............................

LIST OF SYMBOLS / NOMENCLATURE................................................... vii

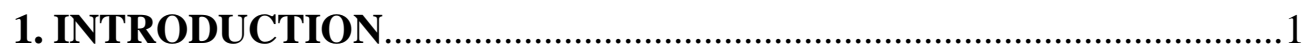

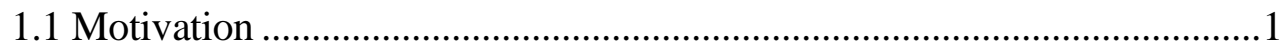

1.2 Delafossite Oxides ....................................................................................

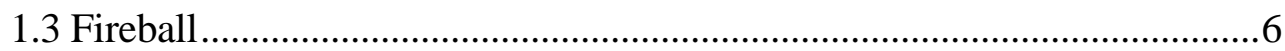

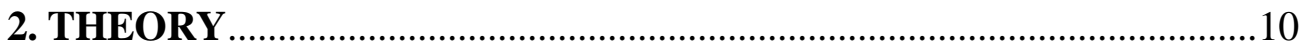

2.1 Enhanced Visible Light Absorption in Delafossites ..................................10

2.2 Benchmarks for Enhanced Visible Light Absorption ...................................14

3. COMPUTATIONAL METHODS ………………….............................

3.1 Creating Models for Simulation..........................................................19

3.1.1 The Primitive Unit Cell ....................................................................19

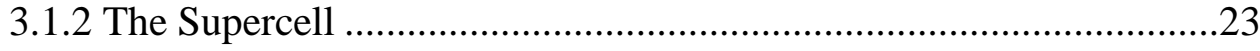

3.2 Electronic Band Structure Calculation..................................................26

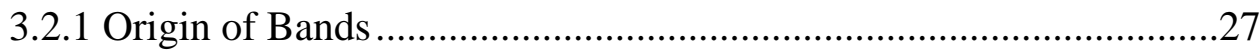

3.2.2 Direct vs. Indirect Transitions..........................................................29

3.3 Electronic Density of States Calcualtion .................................................31

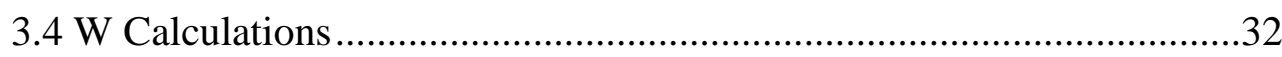

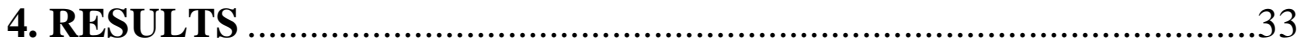

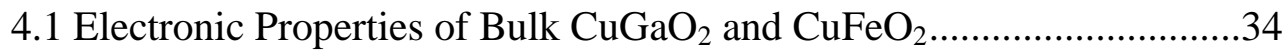

4.2 Electronic Properties of $\mathrm{CuGa}_{1-\mathrm{x}} \mathrm{Fe}_{\mathrm{x}} \mathrm{O}_{2} ; \mathrm{x}=0.05,0.10,0.15,0.20 \ldots \ldots . .38$

4.3 Electronic Properties of $\mathrm{CuGa}_{1-\mathrm{x}} \mathrm{Fe}_{\mathrm{x}} \mathrm{O}_{2 ;} \mathrm{x}=0.01,0.02,0.03,0.04 \ldots \ldots . .45$

5. DISCUSSIONS AND CONCLUSIONS ………………………….......

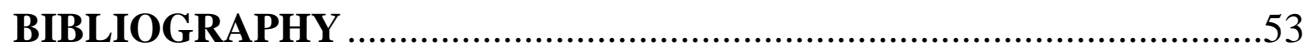




\section{LIST OF FIGURES}

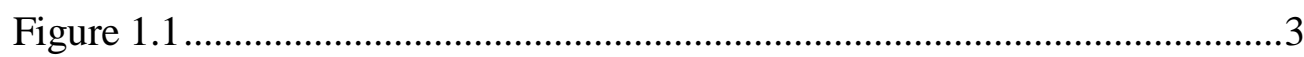

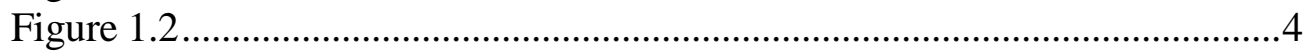

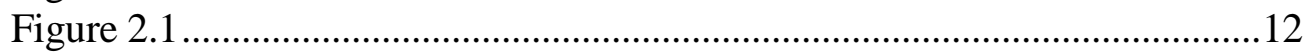

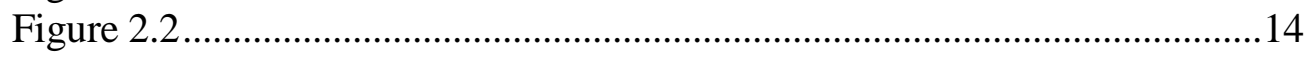

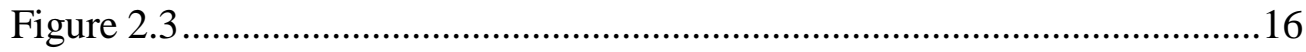

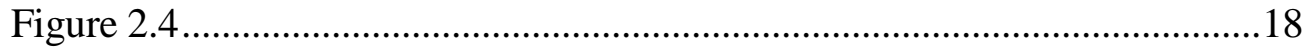

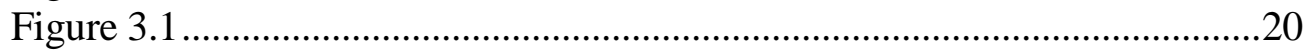

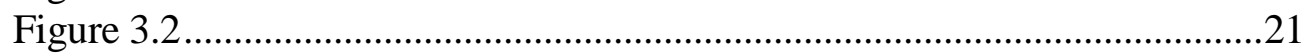

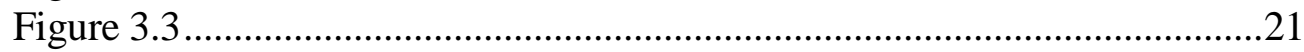

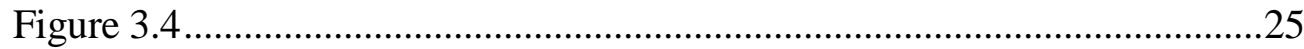

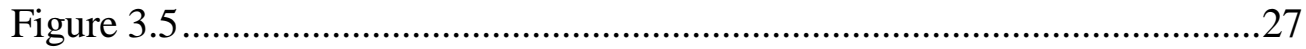

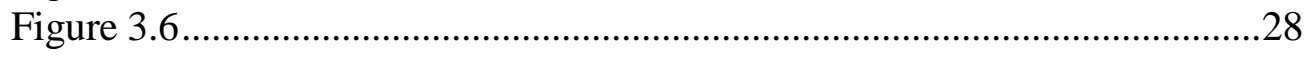

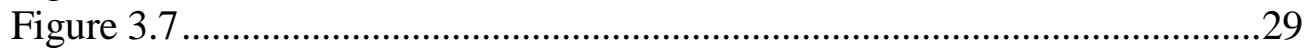

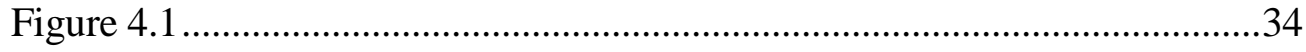

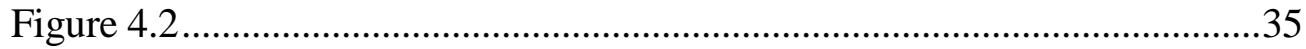

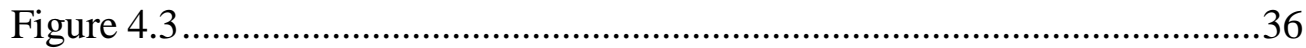

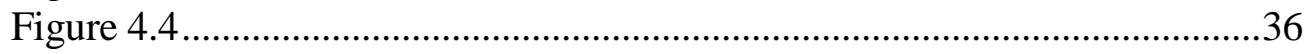

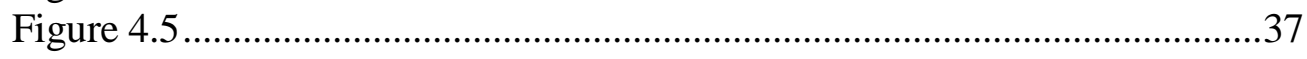

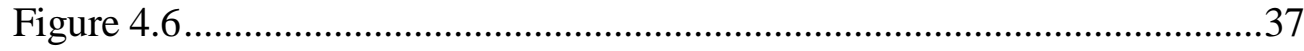

Figure 4.7

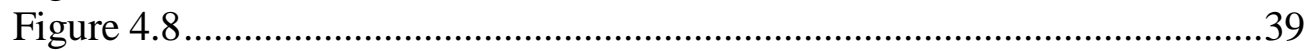

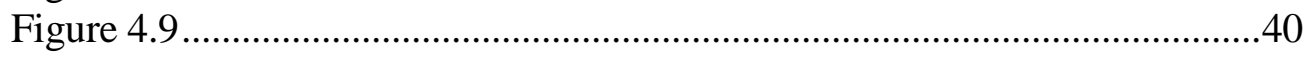

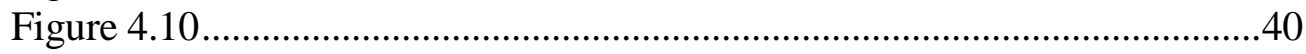

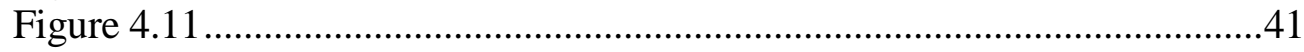

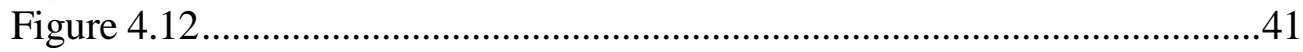

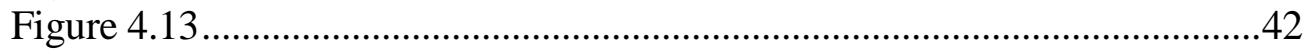

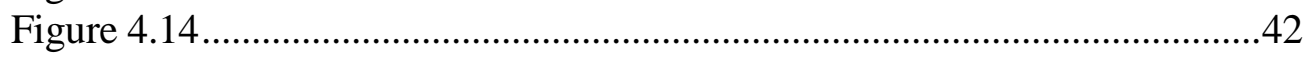

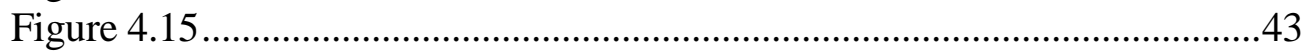

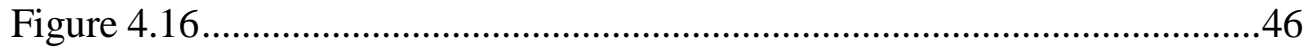

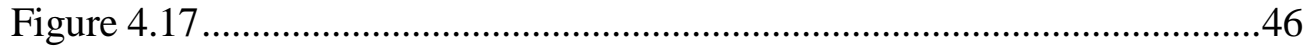

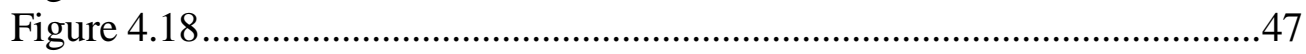

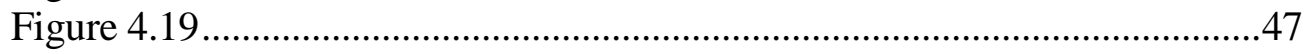

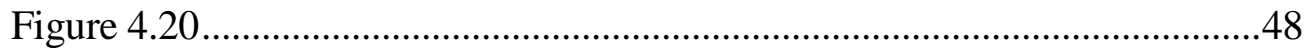

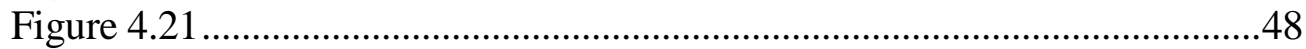

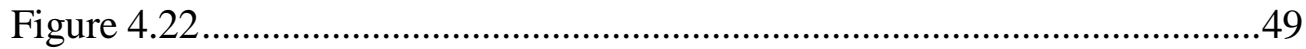

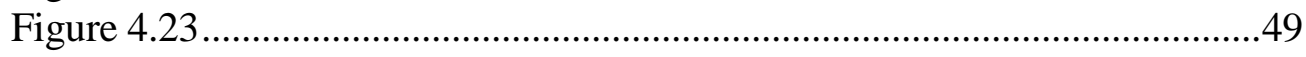

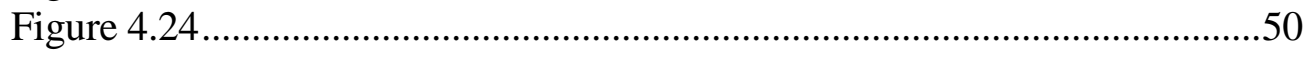




\section{LIST OF TABLES}

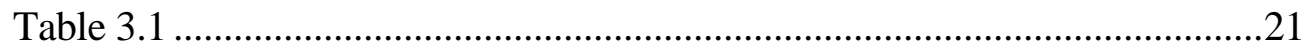

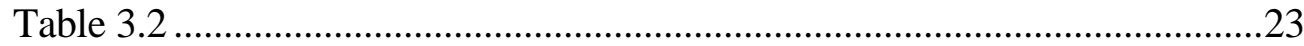

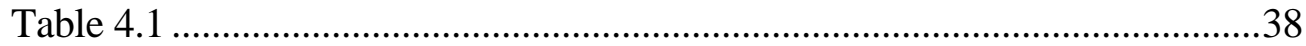

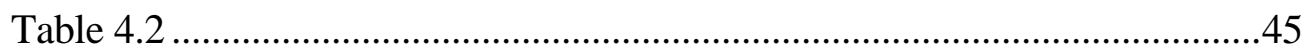


LIST OF SYMBOLS / NOMENCLATURE

PEC - Photoelectrochemical

UV- Ultraviolet

DFT- Density functional theory

QM- Quantum mechanics

EM- Electromagnetic

LCAO- Linear combination of atomic orbitals

MD- Molecular dynamics

CMB- Conduction band minimum

VBM- Valance band maximum

DOS- Density of states

PDOS- Partial density of states

$\mathrm{E}_{\mathrm{F}}$ - Fermi energy

$\mathrm{E}_{\mathrm{MB}}$ - Mid-band energy

$\mathrm{E}_{\mathrm{T}}-$ Energy of trap state

HOMO - Highest occupied molecular orbital

LUMO - Lowest unoccupied molecular orbital 


\section{Introduction}

\subsection{Motivation}

Fossil fuel energy production is essential to our economy as we know it. In the United States alone, there are over 1500 coal-fired power plants which are responsible for affordably producing about $50 \%$ of the energy consumed. However, there is an ugly side to this means of power generation. Nearly $33 \%$ of $\mathrm{CO}_{2}$ emissions in the US are produced by coal-fired power plants alone ${ }^{1}$. This green-house gas threatens our air-quality while increasing global warming effects and thus is a target for emission reduction. Many currently studied methods of carbon remediation such as sequestration are costly due to the need to isolate, capture, transport, and store the $\mathrm{CO}_{2}$. Apart from that, the methods of storage, whether underground or in ocean waters, hold potentially unknown environmental threats ${ }^{2}$.

This research is driven by a need to find a more feasible and cost effective method for carbon remediation. We look toward the developing technology of certain photoelectrochemical cells which can use a photocatalyst combined with solar energy to perform a chemical reduction of a material - in our case, we are interested in the reduction of $\mathrm{CO}_{2}$. In particular, we are interested in reduction reactions that produce the desired products, methanol and methane.

$$
\begin{gathered}
\mathrm{CO}_{2}+6 \mathrm{H}^{+}+6 \mathrm{e}^{-} \rightarrow \mathrm{CH}_{3} \mathrm{OH}+\mathrm{H}_{2} \mathrm{O} \\
\mathrm{CO}_{2}+8 \mathrm{H}^{+}+8 \mathrm{e}^{-} \rightarrow \mathrm{CH}_{4}+2 \mathrm{H}_{2} \mathrm{O}
\end{gathered}
$$

We require a material for this photocatalyst that is stable in aqueous solution with band

edge potentials that straddle the reduction energies of the reactions in equations 1.1 and $1.2^{3}$. This requirement is in addition to the obvious desired property of light absorption in the visible 
spectrum. If we can find a suitable catalyst, it is promising that a device could be designed to capture $\mathrm{CO}_{2}$ from the gas flue of a coal-fired or other fossil fuel based power plant and uses readily available sunlight energy to reduce $\mathrm{CO}_{2}$ into usable products such as methanol and methane. These products could then be sold back into the product stream as alternative fuels. This method would reduce the net cost of pursuing carbon remediation. Although other catalysts have been explored for this purpose ${ }^{4}$, we examine the promising family of oxides known as delafossites to find a more suitable option. 


\subsection{Delafossite Oxides}

The Delafossite family of oxides gathers its name from the material $\mathrm{CuFeO}_{2}$ which was discovered in 1873 by Charles Friedel and is named after Gabriel Delafosse ${ }^{5}$. These materials have the general formula $\mathrm{ABO}_{2}$ where $\mathrm{A}$ is a d ${ }^{10}$ coinage metal cation such as $\mathrm{Cu}^{+}$or $\mathrm{Au}^{+}$and $\mathrm{B}$ is $\mathrm{a}^{+} 3$ metal cation, for example $\mathrm{Ga}^{3+}$ or $\mathrm{Fe}^{3+}$. Depending on the B-site element, the ground state crystalline structure will take on one of two forms: hexagonal with symmetry space group $\mathrm{P}_{3} / \mathrm{mmc}$, or rhombohedral with symmetry space group $\mathrm{R} \overline{3} \mathrm{~m}$. These two forms are shown in Figure 1.1. It was shown by Huda that delafossites with B-site elements belonging to group IIIA on the periodic table $(\mathrm{Ga}, \mathrm{In})$ tend to form rhombohedral structures while structures with group IIIB elements (Sc, Y) in the B-site settle into a hexagonal geometry ${ }^{6}$.

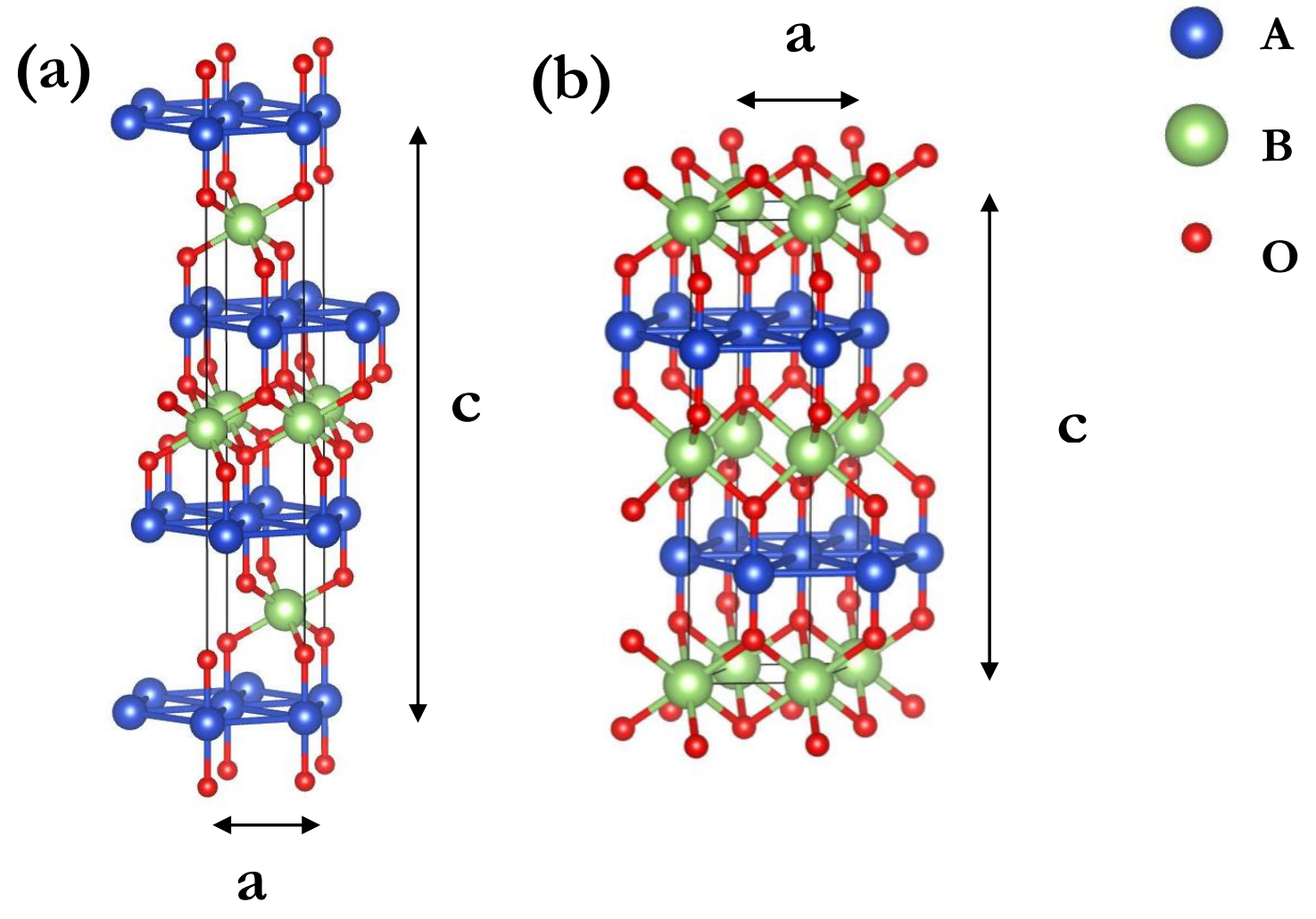

Figure 1.1. The two general $\mathrm{ABO}_{2}$ delafossite structure polytypes. (a) $\mathrm{R} \overline{3} \mathrm{~m}$ symmetry group (b) $\mathrm{P}_{3} / \mathrm{mmc}$ symmetry group. The lattice constants $a$ and $c$ are shown for both structures. 
Delafossites are interesting materials for photoelectrochemical (PEC) applications due to their existence as both p-type $\left(\mathrm{CuAlO}_{2}\right)$ and n-type $\left(\mathrm{AgInO}_{2}\right)$ semi-conductors. They also exhibit excellent hole mobility as well as stability in aqueous solution ${ }^{7}$. These materials are currently of interest for use as transparent conducting oxides (TCOs) ${ }^{8}$ because most delafossite materials have a measured optical band gap that corresponds to the ultraviolet (UV) portion of the electromagnetic spectrum, meaning that these materials in their pure states are transparent to visible light. These materials are of interest to us because they exhibit a forbidden transition from the highest occupied molecular orbital (HOMO) to the lowest unoccupied molecular orbital (LUMO) at the gamma $(\Gamma)$ point. This is the transition of lowest total energy between the occupied and unoccupied energy states. Therefore the optically measured bandgap, or the measured energy needed to promote an electron from the valence band to the conduction band, is much larger than the fundamental band gap as shown for Cu based delafossites by Nie, Wei and Zhang as shown in Figure $1.2^{7 b}$.

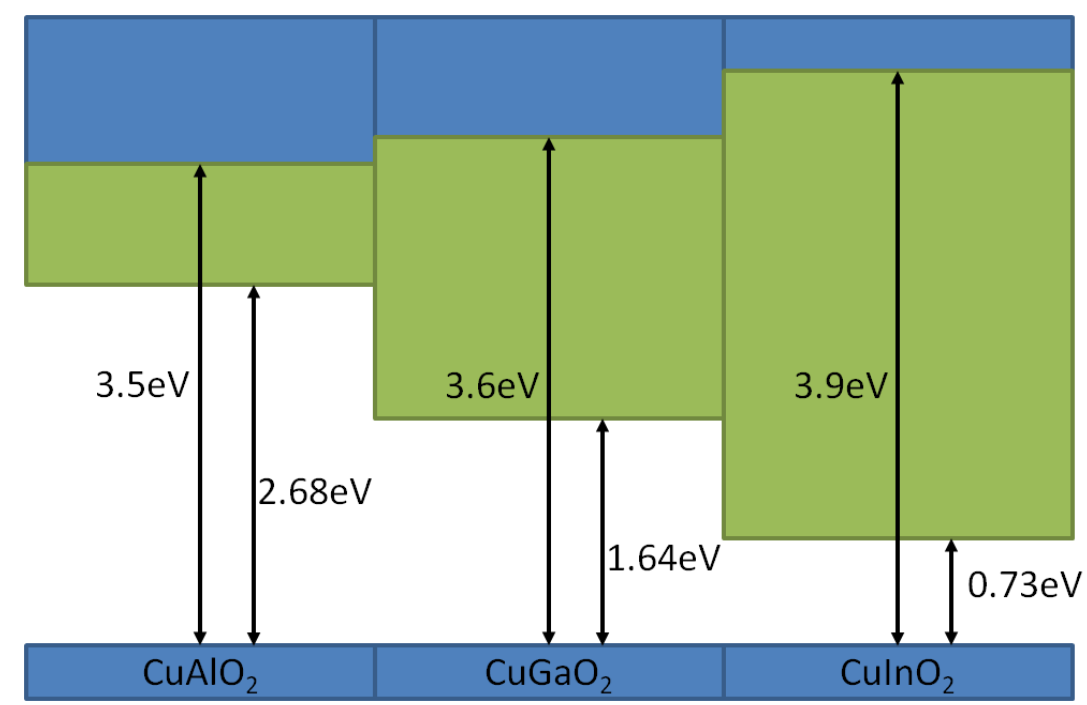

Figure 1.2. Cu-based delafossites have optical bandgaps which are much larger than their fundamental band gaps because the parity of the conduction and valence bands are the same, resulting in a forbidden transition 
We define the fundamental band gap energy as the energy difference between the HOMO and LUMO at the $\Gamma$-point. This direct transition from the valance band to the conduction band at the $\Gamma$-point is disallowed due to the inversion symmetry of the material ${ }^{7 b}$. This research is an attempt to design a delafossite material to overcome this forbidden direct band gap transition and thus allow the utilization of visible light for this material. We make an attempt at engineering the band gap of these delafossite materials by B-site doping. B-site doping is the replacement of the Bsite element with another $3^{+}$metal ion and has been shown by Huda to break inversion symmetry and allow an electronic transition at the fundamental band gap ${ }^{9}$. This method garners multiple different structure possibilities and we hope to find one such combination material with the right properties to reduce $\mathrm{CO}_{2}$ into methane using light from the solar spectrum. Using the computational methods provided by FIREBALL, we model and characterize these materials to understand the electronic effects of B-site doping. 


\subsection{FIREBALL}

The majority of computational calculations reported in this paper were performed using the $a b$ initio tight-binding software package, FIREBALL. The term $a b$ initio means "from first principles" and refers to the fact that no empirical, or experimentally determined, data or special models beyond those derived from the basic and established laws of nature, such as electromagnetic (EM) theory and quantum mechanics (QM) are used in computation. This software takes positional and compositional information about a structure as initial input and solves the Schrödinger Equation for this structure which can be written

$$
H \Psi=E \Psi
$$

Here $H$ is the Hamiltonian of the structure, $E$ is the energy or energy eigenvalues, and $\Psi$ is wave function or energy eigenvectors. For any system, the Hamiltonian can be written

$$
H=T+U
$$

where $\mathrm{T}$ is kinetic energy of the system and $\mathrm{U}$ is the potential energy.

For a system of atoms which consist of nuclei and electrons, we can expand this Hamiltonian further under the Born-Oppenheimer approximation as shown in Equation $(1.5)^{10}$. This approximation states that while the electron wavefunction depends on both the momenta and positions of the electrons, only the positions of the nuclei contribute to the wavefunction. This is derived from the decoupling of the motion of the electrons from the motion of the nuclei due to the fact that a proton is 1836 times more massive than an electron. We approximate no contribution to the kinetic energy from the nuclei as these particles are relatively stationary due to their mass.

$$
H=-\sum_{i}^{N} \frac{\hbar^{2}}{2 m} \nabla_{\mathrm{i}}^{2}+\sum_{\mathrm{i}}^{\mathrm{N}} V\left(\overrightarrow{r_{l}}\right)+\sum_{\mathrm{i}<j}^{\mathrm{N}} \mathrm{U}\left(\overrightarrow{\mathrm{r}}_{\mathrm{l}}, \overrightarrow{\mathrm{r}_{\mathrm{j}}}\right)
$$


The first term is the kinetic energy of the electrons where the summation is over the $\mathrm{N}$ elections and $\nabla_{\mathrm{i}}{ }^{2}$ is the Laplacian for the $\mathrm{i}^{\text {th }}$ atom. The second term describes the potential between the electron and the nuclei where for each atom $i$, where $V\left(\vec{r}_{l}\right)=\sum_{\alpha} \frac{z_{\alpha} e}{r_{i \alpha}}$ and $Z_{\alpha}$ is the charge of nucleus $\alpha, e$ is the elementary charge, and $r_{i \alpha}$ is the distance between atom $i$ and nuclei $\alpha$. The final term in the Hamiltonian describes the interaction between electrons where $\overrightarrow{r_{1}}$ and $\overrightarrow{r_{j}}$ are the positions of the $\mathrm{i}^{\text {th }}$ and $\mathrm{j}^{\text {th }}$ electrons. It is because of the complexity of this last interaction term, that the Hamiltonian cannot be solved exactly for many-body systems ${ }^{11}$. For this reason, FIREBALL uses what is called density functional theory (DFT) ${ }^{12}$. This is an approximation in which we replace the individual electron position dependent exchange correlation energy with a simpler and solvable functional of the electron density. Nearly every modern ab initio molecular dynamics software package uses some form of this DFT approximation.

To solve the Schrödinger equation, you must not only understand the Hamiltonian, but you must choose a way to describe the wavefunction by defining a basis set. A basis set is a set of computationally approximated wavefunctions, linear combinations of which describe the set of physical electronic wave functions. There are two common ways to do this. In the first method, the basis set is defined by a large number of plane waves of the form $e^{i k r}$, where $k$ is the wave vector. To get accurate results for large systems which describe large numbers of electrons, the number of plane waves in the basis must be very large. For systems like the delafossite, getting results using this method is extremely computationally intensive ${ }^{11 b}$. This form of basis set is used by VASP ${ }^{13}$ and ABINIT ${ }^{14}$ to name two of the more well-known computational packages. The second approach is to use a basis set of atomic orbitals which is known as linear combination of 
atomic orbitals (LCAO) ${ }^{11 b}$. This approach is used by FIREBALL ${ }^{15}$ and SIESTA ${ }^{16}$. The number of orbitals needed for large systems depends on the number and types of elemental species in the model rather than the size of the model. FIREBALL treats the local atomic orbitals a bit differently than some other programs by using an ab inito Sankey-Niklewski SN method ${ }^{17}$ rather than empirical counterparts such as LAMMPS ${ }^{18}$. It defines for each orbital a definite cutoff radius rather than allowing the wavefunction of each orbital to trail off to infinity ${ }^{19}$. This creates a confinement for the electron equivalent to the "particle in a box" boundary conditions and thus slightly increases the energy of the state. These slightly excited spherical wavefunctions are referred to as 'atomic fireballs'. This approximation actually assists in calculations of solids as it mimics Fermi-compression behavior ${ }^{20}$. When electrons are in close proximity, as in a solid, their infinite wavefunctions are compressed and thus slightly excited by the presence of other electrons.

Using the constrained atomic wavefunctions of the defined basis set and the DFT approximation, the exchange correlation term of the Hamiltonian can be addressed, Now the Harris-Foulks functional ${ }^{21}$ can be expanded using $\operatorname{McWEDA}^{19}$ as a linear combination of multicenter interactions. Here we show the form of the exchange correlation density functional expansion up to three centers.

$$
V_{X C}(\rho)=\sum_{i} V_{X C_{1}}\left(\rho_{i}\right)+\sum_{i, j} V_{X C_{2}}\left(\rho_{i}, \rho_{j}\right)+\sum_{i, j, k} V_{X C_{3}}\left(\rho_{i}, \rho_{j}, \rho_{k}\right)+\ldots
$$

Here $V_{X C_{1}}$ is the functional of one electron density $\rho_{i}, V_{X C_{2}}$ is the functional of two electron densities $\rho_{i}$ and $\rho_{j}$, and so on. Each of these $V_{X C}$ terms is a complex exchange correlation integral between multiple orbitals. Because these orbitals are well defined, the integrals between them can be pre-calculated using a numerical grid. The one, two and three center interaction integrals are calculated and stored in a folder called 'Fdata'. Four center interactions and beyond are omitted as 
their contribution to the exchange correlation is negligible for our purposes. This means that each integral must only be performed once, and that these integrations take place separately from the optimization process. During the simulation while the solutions to the Schrödinger equation are found, the solutions to the exchange correlation are simply looked up from the Fdata. This dramatically increases the speed of the optimization and enables FIREBALL to evaluate large systems that other similar ab initio programs cannot handle ${ }^{11 a}$.

FIREBALL calculates the forces on each atom and shifts the atoms accordingly in time with the goal of minimizing the force on each atom as well as the energy of the structure as a whole. In addition, FIREBALL can use self-consistent methods such as DOGS ${ }^{15 a, 22}$, which is a reformulation of the non-self consistent Harris-Foulks functional. A self-consistent method is time-independent and optimizes the density of the electrons with respect to the atomic positions between the timebased molecular dynamics steps. The molecular dynamics steps optimize the atomic positions to minimize forces felt within the system. By optimizing the structure through energy minimization, we obtain a ground state configuration that is consistent with the variation theorem. ${ }^{11 \mathrm{~b}}$ The variation theorem states that the approximated calculation of some property of a material is greater than or equal to the actual ground state value of that property. 


\section{Theory}

\subsection{Light Absorption in Delafossites}

Although delafossites are currently studied for use as transparent conducting oxides due to their wide optically measured direct bandgaps $\left(\mathrm{CuAlO}_{2} \sim 3.5 \mathrm{eV}, \mathrm{CuGaO}_{2} \sim 3.6 \mathrm{eV}, \mathrm{CuInO}_{2} \sim\right.$ $3.9 \mathrm{eV})$, the fundamental band gaps of delafossites are much smaller $\left(\mathrm{CuAlO}_{2} \sim 2.68 \mathrm{eV}, \mathrm{CuGaO}_{2}\right.$ $\left.\sim 1.64 \mathrm{eV}, \mathrm{CuInO}_{2} \sim 0.73 \mathrm{eV}\right)^{7 \mathrm{~b}}$. The fundamental direct band gaps are forbidden due to inversion symmetry in the material. The Laporte selection rule ${ }^{23}$ states that in a material exhibiting inversion symmetry, two wavefunctions must be of the opposite parity (odd or even) for an electric dipole transition to take place between them. For example, let us consider two wavefunctions $\Psi_{\mathrm{a}}$ and $\Psi_{\mathrm{b}}$ which both exhibit some parity, either be even or odd. The electric dipole operator is defined by

$$
\hat{d}=\sum_{i} e r_{i}
$$

where $e$ is the elementary charge and $r_{i}$ is the position of the $\mathrm{i}^{\text {th }}$ electron. The probability for an electric dipole transition between the wavefunctions $\Psi_{\mathrm{a}}$ and $\Psi_{\mathrm{b}}$ is defined as follows.

$$
P=\left|\left\langle\Psi_{\mathrm{a}}\left|\sum_{i} e r_{i}\right| \Psi_{\mathrm{b}}\right\rangle\right|^{2}
$$

Because the electric dipole operator is an odd function, when it acts on $\Psi_{\mathrm{b}}$ it results in an answer of opposite parity from $\Psi_{\mathrm{b}}$. Thus, if $\Psi_{\mathrm{a}}$ and $\Psi_{\mathrm{b}}$ are of the same parity, the result is zero. Thus if two wavefunctions in a material with inversion symmetry have the same parity, the transition between them is forbidden. This is the case with the relationship between the highest occupied molecular orbital (HOMO) and lowest unoccupied molecular orbital (LUMO) in delafossite materials.

Delafossites exhibit qualities that are desirable for photoelectrochemical (PEC) applications such as stability in aqueous solutions and long carrier lifetimes. ${ }^{6}$ Therefore, it is prudent to 
understand how modifying the symmetry of these structures can affect their optical band gap. This understanding could lead to the ability to engineer the band gap to selectively absorb desired wavelengths of light, such as the range of the abundant visible solar spectrum.

Previous research by Huda has shown that it is possible to break the inversion symmetry in delafossite materials through B-site alloying thus allowing the fundamental direct band gap transition to take place ${ }^{9}$. This was shown by modeling a $\mathrm{CuYO}_{2}$ structure with $50 \%$ substitution of $\mathrm{Y}$ atoms with $\mathrm{Ga}$ atoms to create $\mathrm{Cu}(\mathrm{Ga}: \mathrm{Y}) \mathrm{O}_{2}$, as shown in Figure 2.1. The structure was made such that $\mathrm{Y}$ and $\mathrm{Ga}$ atoms are not found in the same octahedron layers, but alternate each layer. The alloy was found to decrease the conduction band minimum (CBM) and to preserve the valance band maximum (VBM) of $\mathrm{CuYO}_{2}$ which is essential to for efficient PEC applications. These materials retain their crystalline structure despite lattice substitution; hence this type of B-site doping is not likely to introduce recombination centers, which would decrease recombination times of charge carriers and the efficiency of PEC materials.

Huda's calculations consider B-site materials that have two different symmetry preferences: $\mathrm{CuGaO}_{2}$ prefers rhombohedral symmetry, while $\mathrm{CuYO}_{2}$ prefers hexagonal symmetry. It is a concern in regards to the synthesis of the material that one could face the problem of phase separation between the rhombohedral and hexagonal symmetries of the structure. Because of this, we wish to explore the effects of B-site doping in delafossites where the B-site lattice replacement element prefers the same symmetry as the original delafossite structure. 
(a)

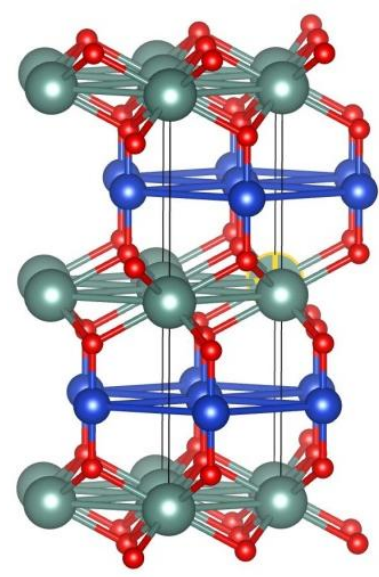

(b)
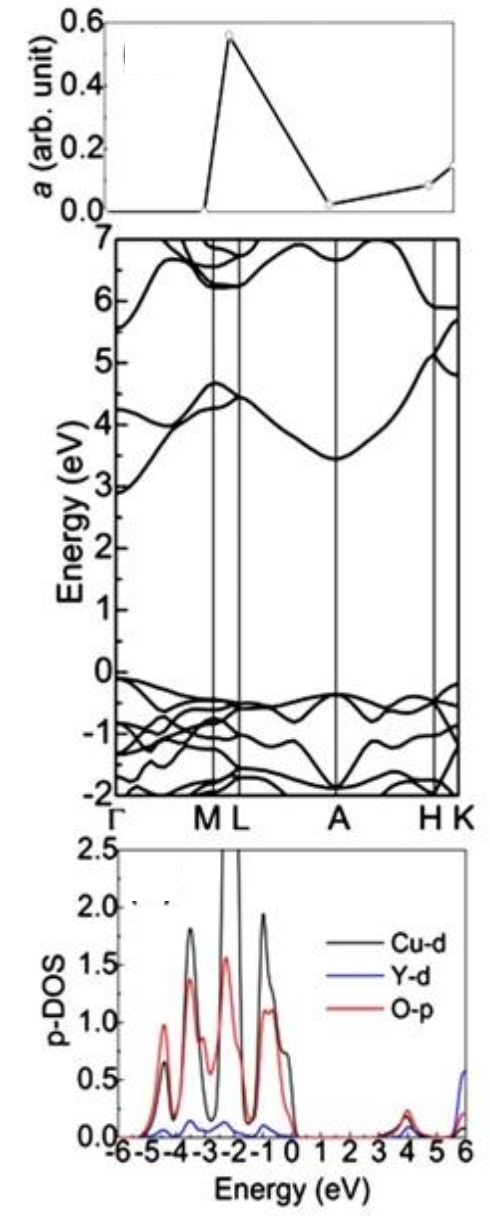

(c)

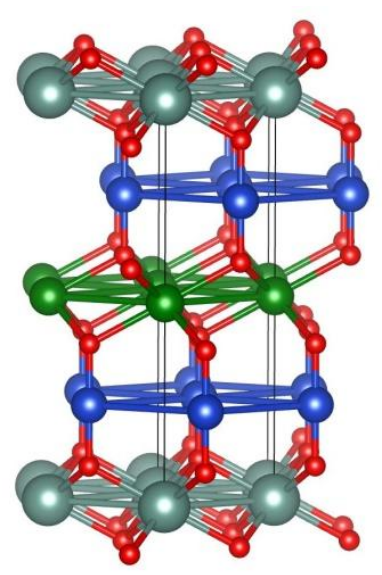

(d)
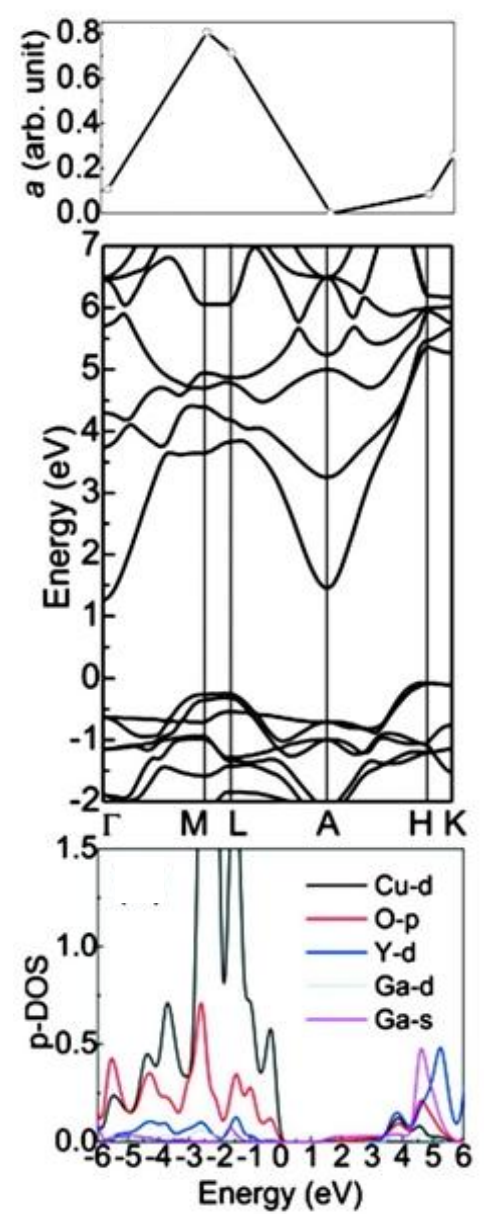

Figure 2.1. (a) $\mathrm{CuYO}_{2}$ structure. (b) Electronic structure data for $\mathrm{CuYO}_{2}$ showing forbidden direct $\Gamma$-point transition (reproduced with permission from [25]).

(c) $\mathrm{Cu}(\mathrm{Y}, \mathrm{Ga}) \mathrm{O}_{2}$ (d) Electronic structure data for $\mathrm{Cu}(\mathrm{Y}, \mathrm{Ga}) \mathrm{O}_{2}$ with broken symmetry and allowed direct $\Gamma$-point transition (Reproduced with permission from $\operatorname{Ref[9]).}$ 
We initially chose to examine $\mathrm{CuGaO}_{2}, \mathrm{CuFeO}_{2}$, and $\mathrm{CuGa}_{1-\mathrm{x}} \mathrm{Fe}_{\mathrm{x}} \mathrm{O}_{2}$ where $\mathrm{x}=0.05,0.10$, 0.15, and 0.20 to understand how this doping with similar B-site materials with respect to ground state symmetry would work. Upon finding that symmetry breaking occurred and little visible light absorption was seen at $\mathrm{x}>0.05$, we chose to study smaller doping percentages where $\mathrm{x}=0.01$, $0.02,0.03$, and 0.04 to understand the conditions necessary and sufficient for symmetry breaking. In this case, both pure delafossite materials exhibit the same preferred symmetry ${ }^{24}$ and similar effective radii ${ }^{25}$ reducing the possibility of phase separation within the synthesized binary material. Different doping percentages were tested to understand how much is sufficient to break the inversion symmetry in the bulk material and allow the fundamental direct band gap. We create these high-percentage doped, or alloyed, computational models by creating a supercell of $\mathrm{CuGaO} 2$ with 192 atoms for $\mathrm{x}=0.05,0.10,0.15$, and 0.20 and with 432 atoms for $\mathrm{x}=0.01,0.02,0.03$, and 0.04. We select the appropriate number of random $\mathrm{Ga}$ atoms and replace them with Fe atoms for the structure of desired doping percentage. The lattice vectors of the structures were scaled linearly with doping percentage according to Vegard's law $^{26}$. Future work will focus on more ordered doping procedures. 


\subsection{Benchmarks for Enhanced Absorption}

Since PEC applications utilize sunlight for energy, it is important to understand the solar spectrum as seen on Earth (Figure 2.2). With the maximum of the solar spectrum at $2.48 \mathrm{eV}$ $(500 \mathrm{~nm})$ and the average energy of a solar photon being $1.35 \mathrm{eV}(919 \mathrm{~nm})$, narrow band gap semiconductors are able to utilize a larger percentage of solar radiation ${ }^{27}$. Therefore, efficiency of a PEC material can be judged partially by the size of the band gap. Reducing the absorption edge of delafossite materials will improve the absorption overlap with the solar spectrum.

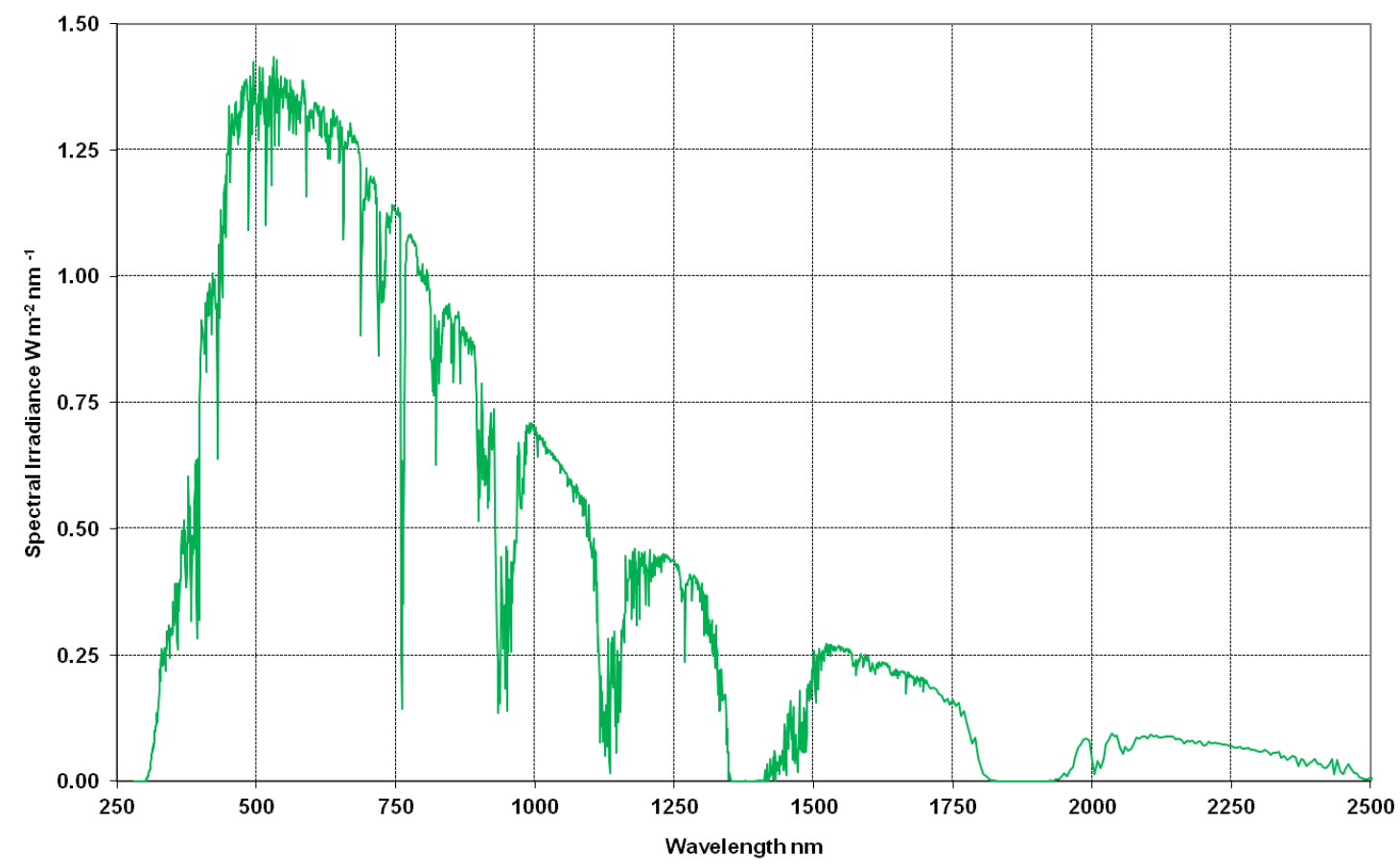

Figure 2.2. ASTM G173-03 reference spectra for direct normal spectral irradiance. 
Sunlight absorption is not enough to qualify a material as a good PEC or photovoltaic material. The production probability of electrons and electron vacancies, known as holes, due to a photonic excitation must be sufficiently high. Electrons and holes, known collectively as carriers, must also have a relatively long lifetime to conduct electricity or catalyze a reaction before recombination. To computationally estimate the carrier times, we must understand two principles: The Shockley-Reed-Hall recombination theory for indirect band gap materials and localization of defects.

The Shockley-Read-Hall recombination theory ${ }^{28}$ describes the recombination times for trapped states for indirect transitions in semiconductors. In our material, the dopant atoms at the B-site add states into the band gap that act as trap states. A trap state is a defect, impurity, or dislocation in a material that traps a carrier and holds it for a period of time before releasing it through some energy transfer. Defining the mid-level energy as halfway between the original conduction and valance band, we can estimate the carrier lifetime by measuring the energy difference $(\Delta \mathrm{E})$ between the trap state energy and the mid-level energy. The greater the $\Delta \mathrm{E}$ between these two states, the longer the carrier lifetime. This is because it is less energetically favorable for an electron to recombine in conduction band. (Figure 2.3) By looking at the electronic band structure calculations for the doped material, we can estimate this $\Delta \mathrm{E}$ to predict which material has the longest carrier lifetimes ${ }^{28}$.

Though a large $\Delta \mathrm{E}$ from the mid-band energy to the defect energy level is desirable, we must optimize this $\Delta \mathrm{E}$ according to the competing mechanism of localization. The more localized the defect state, the lower the electron emission probability. This is because localized defect states are non-radiative recombination centers that trap states the carriers. The carrier capture time is long 
enough that the hole and electron annihilate before reaching their respective band edges. The ideal situation for enhancing carrier lifetime is to have the delocalized defect state far from the mid-level band that acts as a trap state but not as a recombination center.

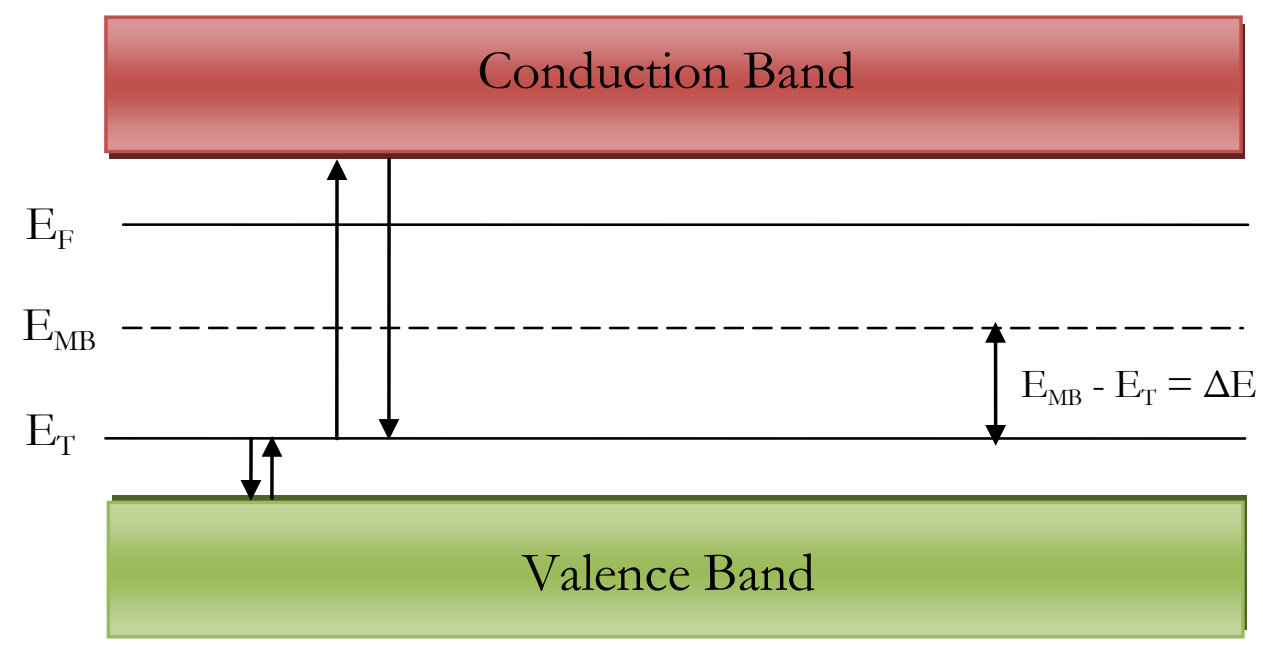

Figure 2.3. Depiction of indirect semi-conductor transition with a trapped state where $\mathrm{E}_{\mathrm{F}}$ is the Fermi energy, $\mathrm{E}_{\mathrm{MB}}$ is the mid-level energy, and $\mathrm{E}_{\mathrm{T}}$ is the energy of the trapped state. Shockley-Read-Hall theory states that maximizing $\mathrm{E}_{\mathrm{MB}}-\mathrm{E}_{\mathrm{T}}$ increases recombination times.

For PEC applications, the positions of the valance and conduction band edges are of utmost importance. For a reduction reaction to take place photoelectrochemically, the band edges (CBM and VBM) of the electrode material must straddle the desired reaction potential. In this case, we look at the reduction of $\mathrm{CO}_{2}$ into methane $\left(\mathrm{CH}_{4}\right)$. This half reaction is found in Eq. 1.2. Since the doping of a material may change the VBM and CBM due to the addition of trap states, discussed earlier, we must assure that the new band edges still sit above and below, straddling the desired reduction reaction ${ }^{29}$. Figure2.4 shows the band edge potentials of common electrode materials for PEC applications as well as the reaction potentials of several common reduction and oxidation 
reactions. The valence band edge of $\mathrm{CuGaO}_{2}$ has been measured to be $-5.1 \mathrm{eV}$ with respect to vacuum ${ }^{8 \mathrm{c}}$ with a bandgap of $3.6 \mathrm{eV}$. This places the conduction band edge at $-1.5 \mathrm{eV}$ with respect to vacuum. The reduction potential of $\mathrm{CO}_{2}$ to $\mathrm{CH}_{4}$ (Eq. 1.2) has a reaction potential of $-0.24 \mathrm{~V}$ with respect to the normal hydrogen electrode at a $\mathrm{pH}$ of $7 .^{30}$ This falls between the VBM and CBM of $\mathrm{CuGaO}_{2}$ which means the $\mathrm{CuGaO}_{2}$ electrode can catalyze the reduction of $\mathrm{CO}_{2}$ to $\mathrm{CH}_{4}$. We wish, however, to minimize the bandgap so that visible light can be utilized. When the minimization of the band gap is achieved, the new CBM and VBM must still straddle the reduction potential of $\mathrm{CO}_{2}$ to $\mathrm{CH}_{4}$ to be considered a valid electrode material for the desired application. . These values are not marked on Figure 2.4 as the figure shows potentials for a $\mathrm{pH}$ of 1 while these are measured at a neutral $\mathrm{pH}$ of 7 . 


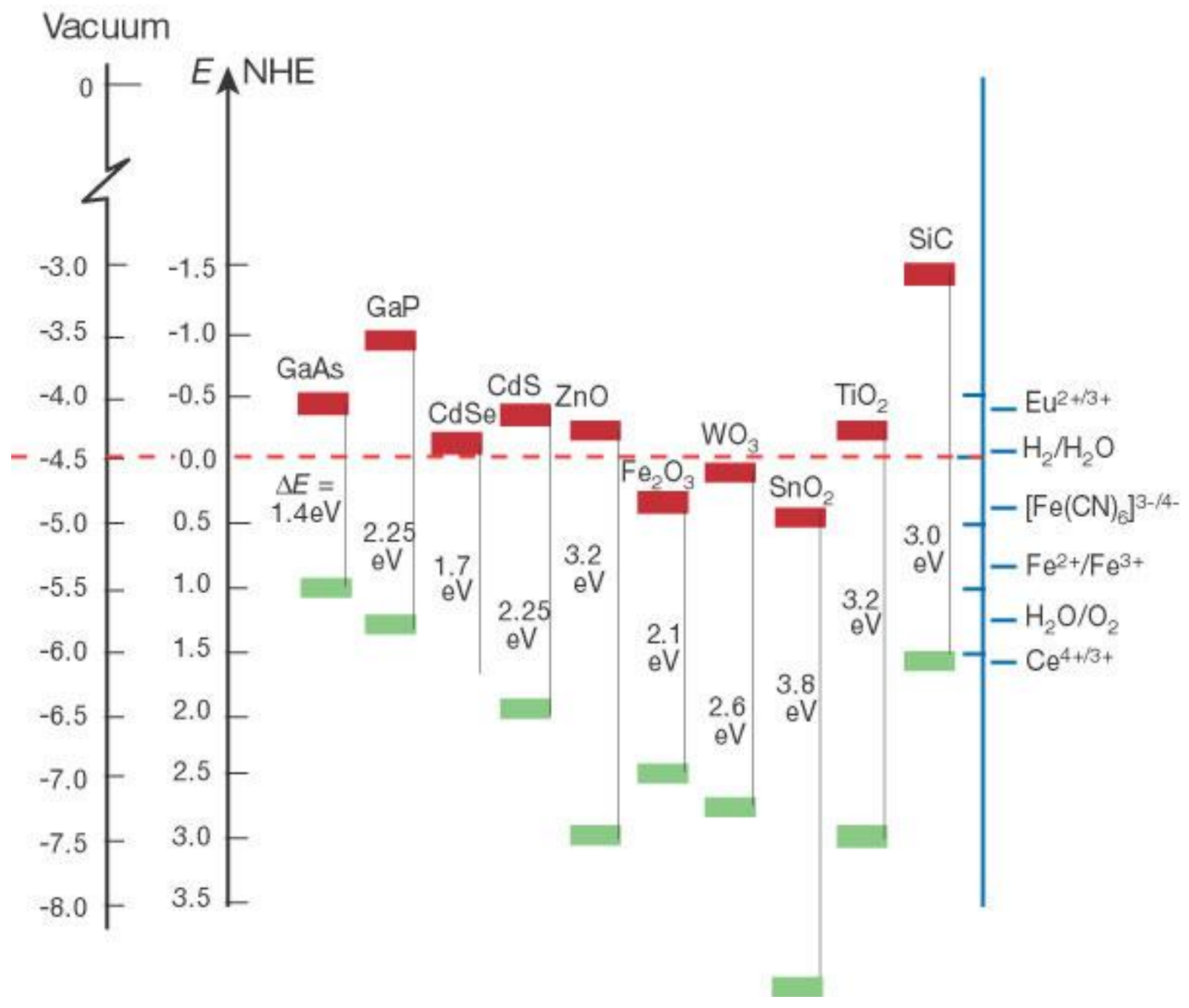

Figure 2.4. Graphical representation of band edge potentials of common PEC electrode materials as well as reaction potentials of common redox reactions. The valance band edge is highlighted in green and the conduction band edge in red. For comparison, $\mathrm{CuGaO}_{2}$ has a valance band edge of $-5.1 \mathrm{eV}$ with an optically measured gap of about $3.6 \mathrm{eV}$ and the reaction potential of $\mathrm{CO}_{2} / \mathrm{CH}_{4}$ is $-0.24 \mathrm{~V}$. Reproduced with permission from $\operatorname{Ref}[29]$ 


\section{Computational Methods}

All computations were performed on one of two machines: the 128-core Nanotech cluster or the 192-core Mountaineer cluster. Both reside at the Chemistry Research Laboratories Building at West Virginia University and serve the state in the newly developed state-wide high performance computing (HPC) initiative.

\subsection{Creating Models for Simulation}

\subsubsection{The Primitive Unit Cell}

To obtain a band structure for a material computationally, one must model the primitive unit cell and then optimize it using periodic boundary conditions. When we discuss the theory of band structures in the next subchapter, we will further explain why this is The $\mathrm{CuGaO}_{2}$ and $\mathrm{CuFeO}_{2}$ structures in the ground state have space group symmetry \#166 or $\mathrm{R} \overline{3} \mathrm{~m}$ which is a more specific symmetry belonging to that of the rhombohedral Bravais lattice. The rhombohedral Bravais lattice has the properties that all sides are equal $(\boldsymbol{a}=\boldsymbol{b}=\boldsymbol{c})$ and all the angles are equal and are not right angles $\left(\boldsymbol{\alpha}=\boldsymbol{\beta}=\boldsymbol{\gamma} \neq 90^{\circ}\right)$. This system can be thought of as a cubic system stretched along one of the body diagonals. Previous work gives experimental lattice constants for the rhombohedral crystal structure of $\mathrm{CuGaO}_{2}$ and $\mathrm{CuFeO}_{2}{ }^{5}$. These are commonly listed as $\boldsymbol{a}$ and $\boldsymbol{c}$ where $\boldsymbol{a}$ is the distance between nearest neighbor $\mathrm{Cu}$ (A-site) atoms and $\boldsymbol{c}$ is the length of the stretched body diagonal of the rhombus. In the primitive cell, all atoms align along the $c$-axis diagonal. The A-site atom $(\mathrm{Cu})$ sits at the lattice points, while the B-site atom $(\mathrm{Ga}$ or $\mathrm{Fe})$ is positioned halfway along the diagonal. The $\mathrm{O}$ atoms are positioned at some internal coordinate $\mathrm{x}$ 
and -x of the way along the diagonal. In $\mathrm{CuGaO}_{2}$ and $\mathrm{CuFeO}_{2}$ this internal coordinate is about 1/9 as is shown in Figure 3.1.

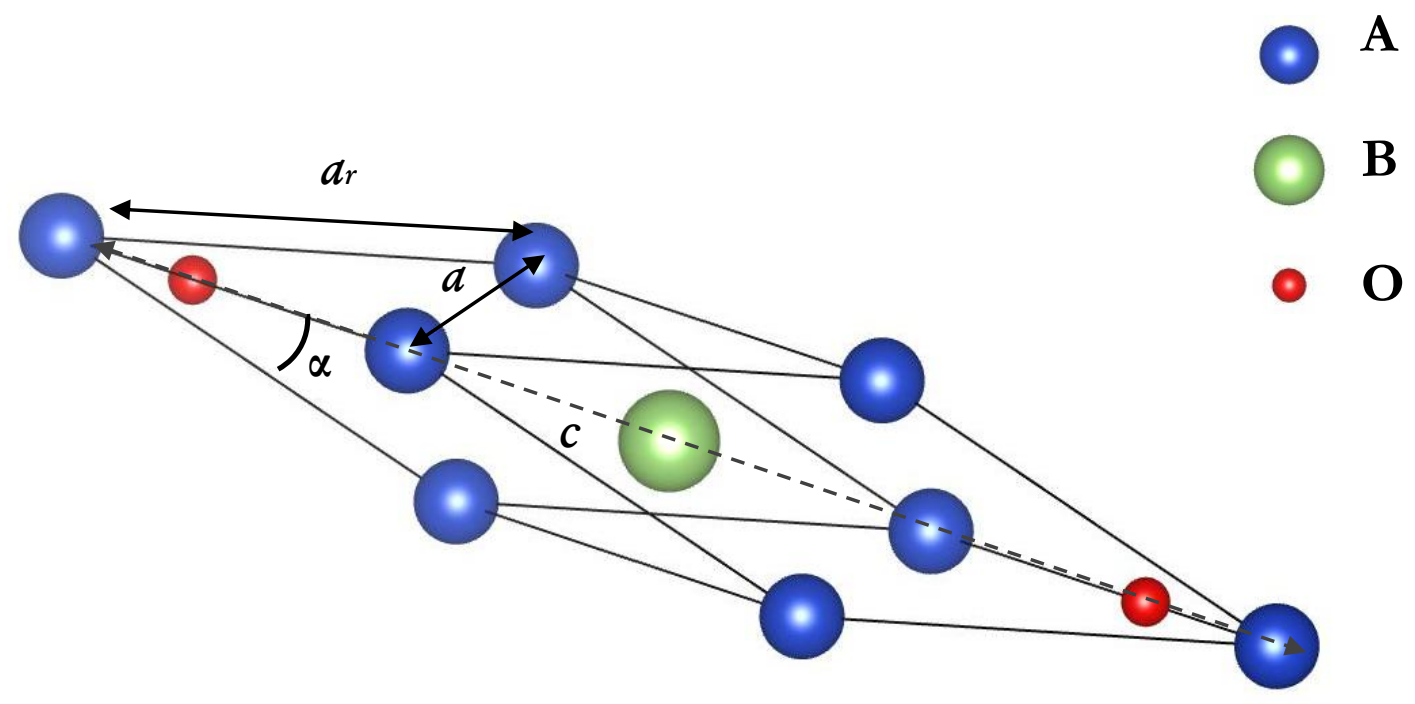

Figure 3.1. Rhombohedral primitive unit cell of a delafossite. Shown are the rhombohedral constants $\boldsymbol{a}_{\boldsymbol{r}}$ and $\boldsymbol{\alpha}$ as well as the equivalent hexagonal constants $\boldsymbol{a}$ and $\boldsymbol{c}$.

The optimized values used for the structures were determined by creating a 21 x 21 "grid" of calculations for each system centered at the experimental $\boldsymbol{a}$ and $\boldsymbol{c}$ lattice constants. The values of the constants were varied by steps of $0.5 \%$ of the original value on either side. Each new structure was optimized. The $\boldsymbol{a}$ and $\boldsymbol{c}$ values were then graphed versus the total energy per atom of the optimized structure as shown in Figures 3.2 and 3.3. These three-dimensional graphs are difficult to represent clearly in two-dimension space. The energy is marked along the z-axis while the $\boldsymbol{a}$ lattice constant is along the x-axis, and the $\boldsymbol{c}$ lattice constant is along the $\mathrm{y}$-axis. The minimum energy structure with $\boldsymbol{a}$ and $\boldsymbol{c}$ values nearest the experimental values was taken to have 
the optimized lattice constants. We chose the structure with the lowest energy in accordance with the variation theorem ${ }^{11 b}$ discussed earlier.

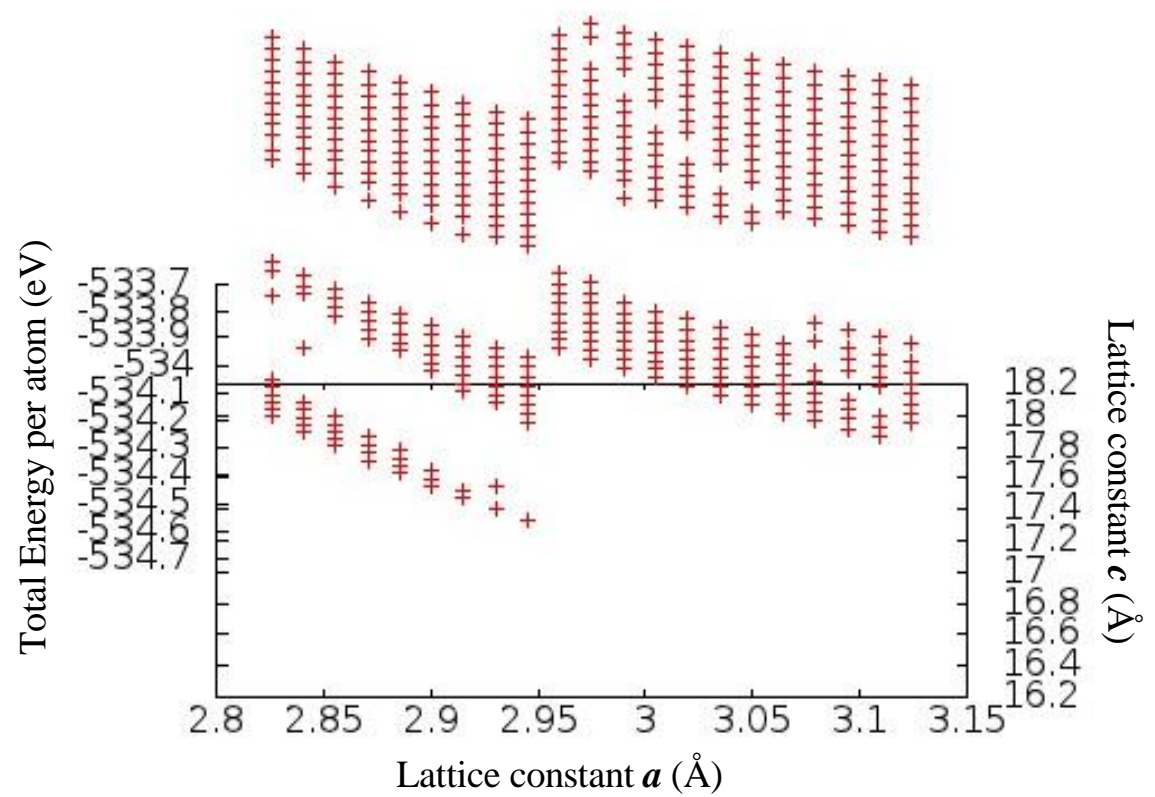

Figure 3.2. The lattice optimization results for $\mathrm{CuGaO}_{2}$. The minimized energy results for each run are graphed with respect to the $\boldsymbol{a}$ and $\boldsymbol{c}$ values used for each calculation. The minimum value nearest the experimental lattice constants in the center of the graph is taken as that with the optimized lattice constants.

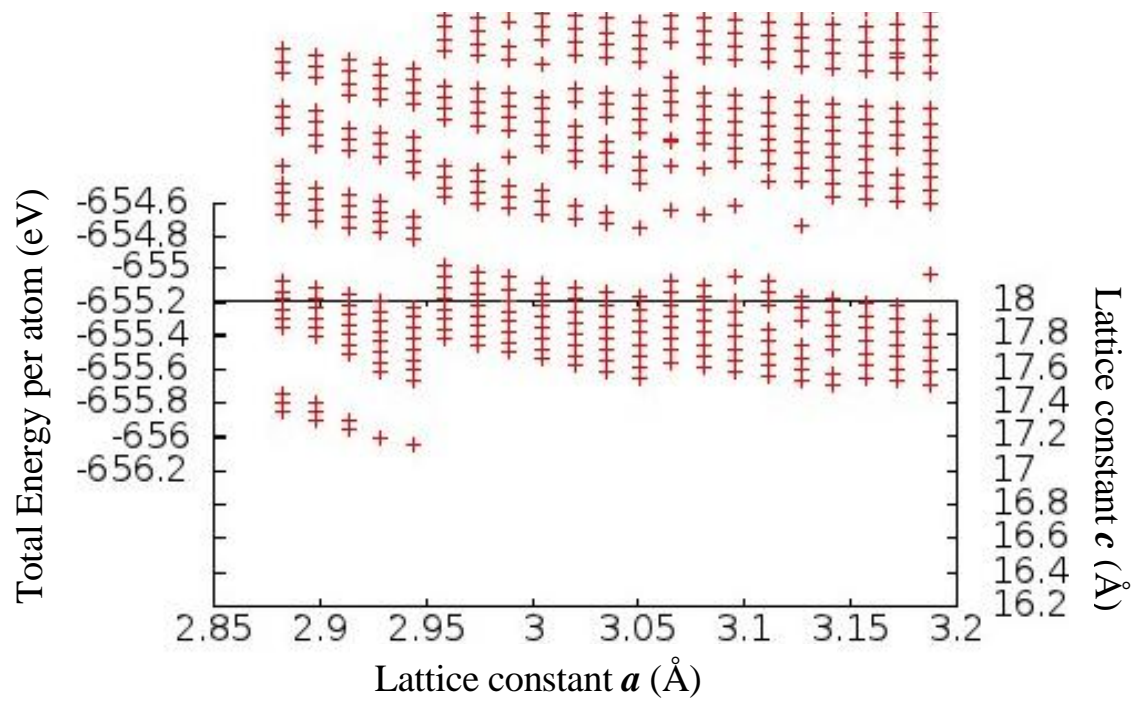

Figure 3.3. The lattice optimization results for $\mathrm{CuFeO}_{2}$. The minimized energy results for each run are graphed with respect to the $\boldsymbol{a}$ and $\boldsymbol{c}$ values used for each calculation. The minimum value nearest the experimental lattice constants in the center of the graph is taken as that with the optimized lattice constants. 
The lattice constants $\boldsymbol{a}$ and $\boldsymbol{c}$ can be converted to the more generic yet equivalent rhombohedral lattice constants $\boldsymbol{a}_{\boldsymbol{r}}$ and $\boldsymbol{\alpha}$ according to the following equations.

$$
\begin{gathered}
a_{r}=\frac{1}{3} \sqrt{c^{2}+3 a^{2}} \\
\alpha=\cos ^{-1}\left(\frac{2 c^{2}-3 a^{2}}{2 c^{2}+6 a^{2}}\right)
\end{gathered}
$$

These new constants are used to determine the $\mathrm{RHL}_{1}$, or rhombohedral unit cell with $\boldsymbol{\alpha}<90^{\circ}$, lattice vectors and special high-symmetry k-points as defined by Setyawan ${ }^{31}$. The experimental and optimized lattice constants $\boldsymbol{a}, \boldsymbol{c}, \boldsymbol{a}_{\boldsymbol{n}}$ and $\boldsymbol{\alpha}$ are shown in Table 1.

\begin{tabular}{|c|c|c|c|c|c|}
\cline { 3 - 5 } \multicolumn{2}{c|}{} & $\boldsymbol{a}$ & $\boldsymbol{c}$ & $\boldsymbol{a}_{\boldsymbol{r}}$ & $\boldsymbol{A}$ \\
\hline $\mathrm{CuGaO}_{2}$ & Experimental & $2.975 \AA$ & $17.154 \AA$ & $5.970 \AA$ & $28.854^{\circ}$ \\
\hline & Optimized & $2.945 \AA$ & $16.982 \AA$ & $5.911 \AA$ & $28.852^{\circ}$ \\
\hline $\mathrm{CuFeO}_{2}$ & Experimental & $3.0351 \AA$ & $17.166 \AA$ & $5.984 \AA$ & $29.380^{\circ}$ \\
\hline & Optimized & $3.0503 \AA$ & $16.909 \AA$ & $5.905 \AA$ & $29.936^{\circ}$ \\
\hline
\end{tabular}

Table 3.1. Primitive rhombohedral unit cell of a $\mathrm{ABO}_{2}$ delafossite with $\mathrm{R} \overline{3} \mathrm{~m}$ lattice constants $\boldsymbol{a}$ and $\boldsymbol{c}$ and more generic rhombohedral lattice constants $\boldsymbol{a}_{\boldsymbol{r}}$ and $\boldsymbol{\alpha}$ shown.

These unit cells were optimizing using the fast quench method of FIREBALL under periodic boundary conditions given by the lattice vectors defined in $\operatorname{Ref}^{31}$. A set of 8 special MonkhorstPack k-points ${ }^{32}$ that include inversion were generated. These k-points define the high symmetry points for the energy optimization. A single point energy calculation was then run on the optimized structure to obtain the energy eigenvalues with respect to k-space using the highsymmetry k-points. 


\subsubsection{The Supercell}

Using the program VESTA (Visualization for Electronic and Structural Analysis) ${ }^{33}$ and the constants given in Table 3.1, we created unit cells (Figure 2.1) with R $\overline{3} \mathrm{~m}$ symmetry with 12 atoms each. These cells were then duplicated in the $\boldsymbol{a}_{1}, \boldsymbol{a}_{2}$, and $\boldsymbol{a}_{3}$ lattice directions to create $4 \times 4 \times 1$ supercells consisting of 192 atoms for the 5\% - 20\% doped structures, bulk $\mathrm{CuGaO}_{2}$ and bulk $\mathrm{CuFeO}_{2}$, and $6 \times 6 \times 1$ supercells consisting of 432 atoms for the $1 \%$ to $4 \%$ doped structures.

To create the B-site doped structures, a $\mathrm{CuGaO}_{2}$ supercell is created and a number of $\mathrm{Ga}$ atoms are randomly selected by a pseudorandom number and replaced with Fe atoms. The lattice constants for each structure are scaled based on Vegard's law ${ }^{26}$ before the pseudorandom replacement with Fe occurs. Supercell structures are shown in Figure 3.4. Vegard's law governs the linear scaling of lattice vectors for doped structures and is written as

$$
x c_{a}+(1-x) c_{b}=c_{n e w}
$$

Here $\mathrm{x}$ is the doping percentage, $\mathrm{c}_{\mathrm{a}}$ is the lattice constant of the structure which is being added or doped into the base structure, and $c_{b}$ is the lattice constant of this base structure. This gives $c_{\text {new }}$ or the lattice constant of the new doped structure. Here we use the lattice constants $\boldsymbol{a}$ and $\boldsymbol{c}$ for $\mathrm{CuGaO}_{2}$ and $\mathrm{CuFeO}_{2}$ and treat the doped structure as an alloy of the two. This simplified the process of calculating new lattice constants since $\mathrm{CuFeO}_{2}$ is of the same crystal symmetry as $\mathrm{CuGaO}_{2}$. We know this is a valid scaling for our structures because experiment has shown a linear scaling of lattice volume with the increased percentage of Fe doped into $\mathrm{CuGaO}_{2}{ }^{34}$.

Due to the discrete nature of the super cell and B-site replacement, the resulting structure differs in exact percentage. The exact percentage is calculated based on the number of Ga atoms replaced 
with $\mathrm{Fe}$ atoms divided by the total number of $\mathrm{Ga}$ atoms in the original structure. The error in percentage for all structures is less than $0.5 \%$ and is shown in Table 3.2.

\begin{tabular}{|c|c|c|}
\hline Structure & $\begin{array}{c}\text { \# of Ga atoms } \\
\text { replaced }\end{array}$ & $\begin{array}{c}\text { Actual } \\
\text { percentage }\end{array}$ \\
\hline $\mathrm{CuGa}_{0.99} \mathrm{Fe}_{0.01} \mathrm{O}_{2}$ & 1 & $0.9 \%$ \\
\hline $\mathrm{CuGa}_{0.98} \mathrm{Fe}_{0.02} \mathrm{O}_{2}$ & 2 & $1.85 \%$ \\
\hline $\mathrm{CuGa}_{0.97} \mathrm{Fe}_{0.03} \mathrm{O}_{2}$ & 3 & $2.8 \%$ \\
\hline $\mathrm{CuGa}_{0.96} \mathrm{Fe}_{0.04} \mathrm{O}_{2}$ & 4 & $3.7 \%$ \\
\hline $\mathrm{CuGa}_{0.95} \mathrm{Fe}_{0.05} \mathrm{O}_{2}$ & 2 & $4.2 \%$ \\
\hline $\mathrm{CuGa}_{0.90} \mathrm{Fe}_{0.10} \mathrm{O}_{2}$ & 5 & $10.4 \%$ \\
\hline $\mathrm{CuGa}_{0.85} \mathrm{Fe}_{0.15} \mathrm{O}_{2}$ & 7 & $14.6 \%$ \\
\hline $\mathrm{CuGa}_{0.80} \mathrm{Fe}_{0.20} \mathrm{O}_{2}$ & 10 & $20.8 \%$ \\
\hline
\end{tabular}

Table 3.2. Number of atoms replaced and the resulting actual percentages for each of the $\mathrm{CuGa}_{1-\mathrm{x}} \mathrm{Fe}_{\mathrm{x}} \mathrm{O}_{2}$ structures where $\mathrm{x}=0.01,0.02,0.03,0.04,0.05,0.10,0.15$, and 0.20 . Due to the discrete nature of the supercells, the doping percentages cannot be exact. 


\section{(a)}

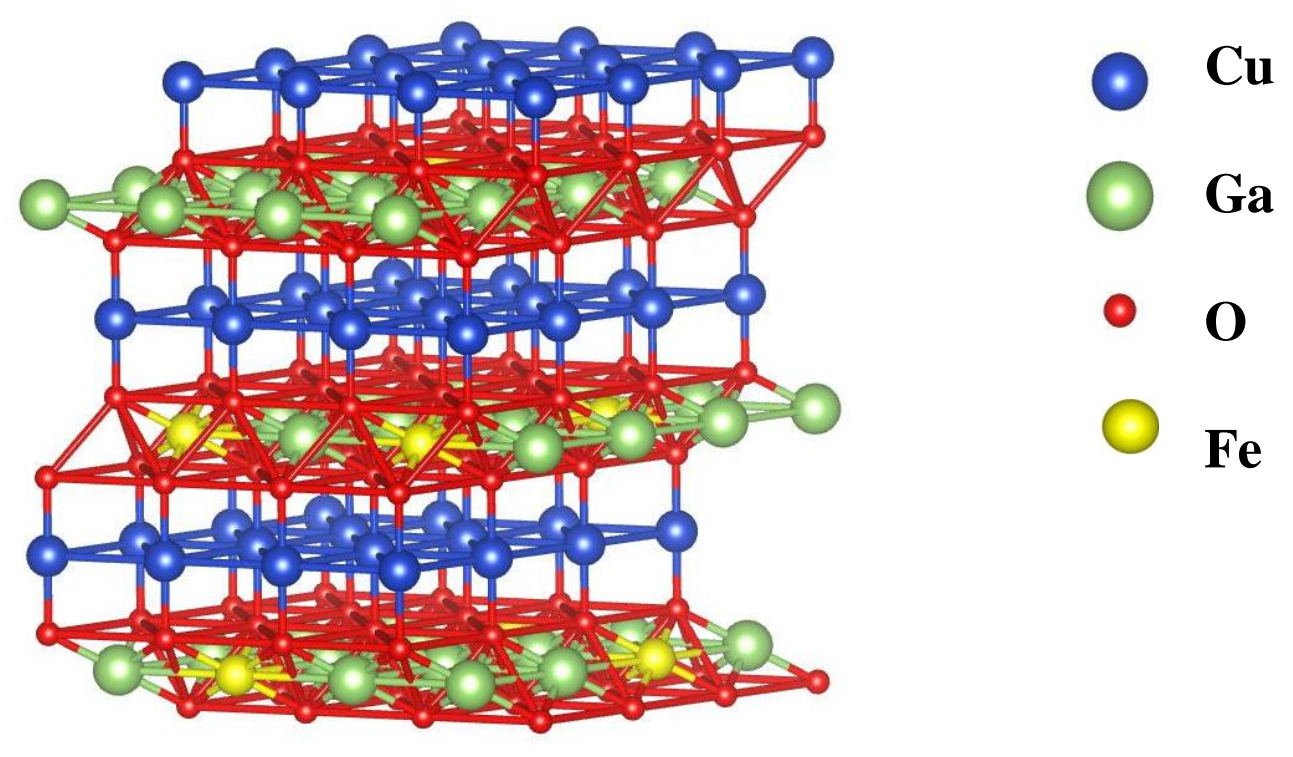

(b)

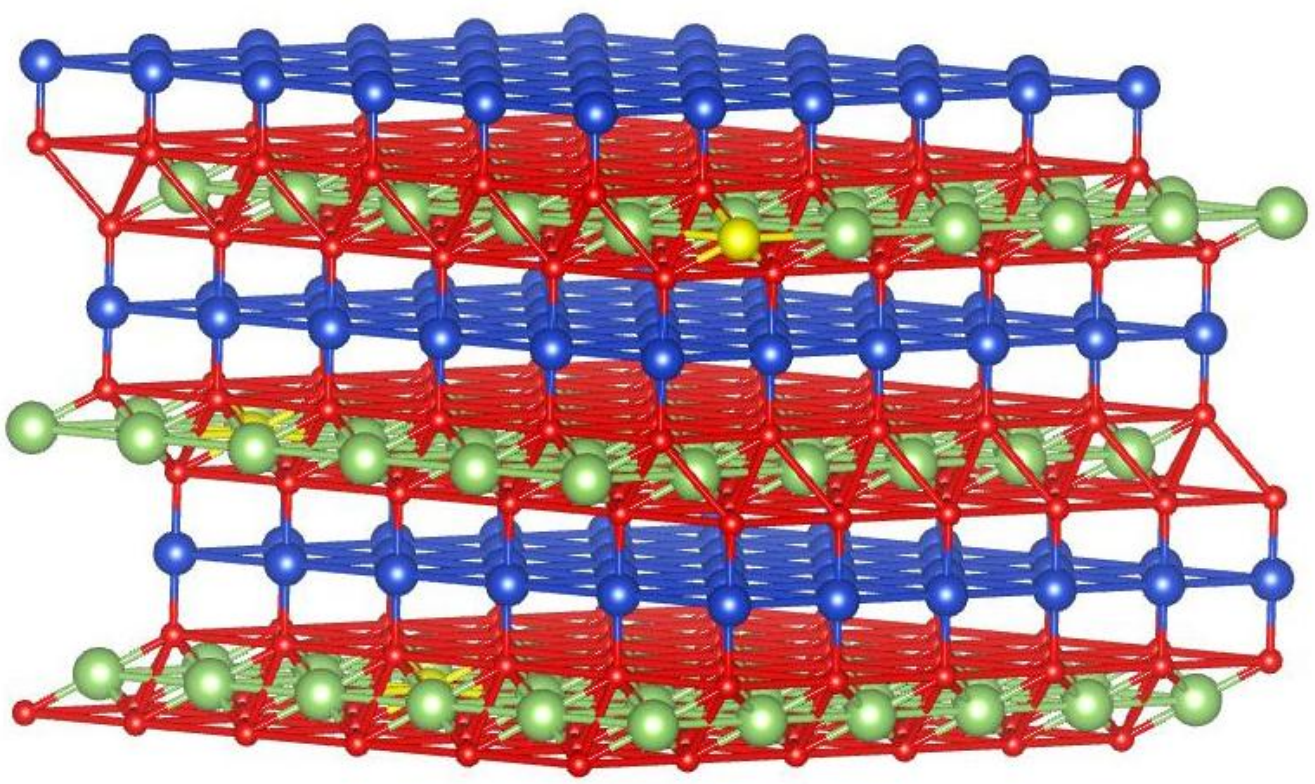

Figure 3.4. (a) $\mathrm{CuGa}_{0.85} \mathrm{Fe}_{0.15} \mathrm{O}_{2}$ 192-atom supercell. (b) $\mathrm{CuGa}_{0.97} \mathrm{Fe}_{0.03} \mathrm{O}_{2}$ 432-atom supercell. 


\subsection{Electronic Band Structure Calculation}

The electronic properties of material arise from the electronic band structure. The band structure graphs the $\mathrm{E}(\boldsymbol{k})$ dispersion of each band, which describe individual molecular orbitals within a certain structure. Here, the wave vector $\boldsymbol{k}$ defines reciprocal space which is the result of Fourier transforming real space, denoted as $\boldsymbol{r}$. Performing a Fourier transform on the primitive or unit cell of a crystal results in the Brillouin zone; this is a reciprocal space representation of the crystal, preserving all of its symmetry operations. Positions in $\boldsymbol{k}$-space also describe vibrations of entire planes of atoms within the crystal since the wave vector can be linked to the momentum in quantum mechanics by $\boldsymbol{\rho}=\hbar \boldsymbol{k}$.

Band structures are useful in a crystalline system because they map the energy of the states in momentum space based on the symmetry of a structure. Energy bands are graphed over wave vectors inside the first Brillouin zone, or Wigner-Seitz cell, which connect high symmetry points in $\boldsymbol{k}$-space also known as $\boldsymbol{k}$-points ${ }^{35}$. We examine the band structures of the pure $\mathrm{CuGaO}_{2}$ and $\mathrm{CuFeO}_{2}$ structures.

We use a one-dimensional tight-binding approach to present the critical components of the electronic band structure calculation. Bloch's theorem describes the periodicity of the lattice wavefunction

$$
\psi^{k}(x+a)=e^{i k a} \psi^{k}(x)
$$

where $a$ is the lattice constant, $x$ is the position in space, and $k$ is the wave vector. The Bloch condition (3.4) is satisfied by the following wavefunction.

$$
\psi^{k}(x)=\sum_{a} e^{i k a} \phi(x-a)
$$

Here $\phi$ is a linear combination of atomic orbitals defined 


$$
\phi(x)=\sum_{n} b_{n} \psi_{n}(x)
$$

The bands can then be described as the $\boldsymbol{k}$ dependent energy solutions $\mathrm{E}(\boldsymbol{k})$ to the Schrödinger equation.

$$
H \psi^{k}(x)=E(k) \psi^{k}(x)
$$

\subsubsection{Origin of Bands}

Conceptually, one can think of energy bands in the following way. Energy levels in molecules are discrete and form from the hybridization of energy levels in individual atoms. This is known as Molecular Orbital theory and was first described by Mulliken, Hund, and LennerdJones in the late $1920{ }^{3} \mathrm{~s}^{36}$. In a solid which consists of many atoms in close proximity to each other these energy levels become so close together that they are nearly continuous over a range of allowed energies as shown in Figure 3.5. Between these ranges of allowed energies, there sometimes lie areas in the energy where there exists no molecular orbital. These energy regions which are forbidden to the electrons within the structure are called band gaps.

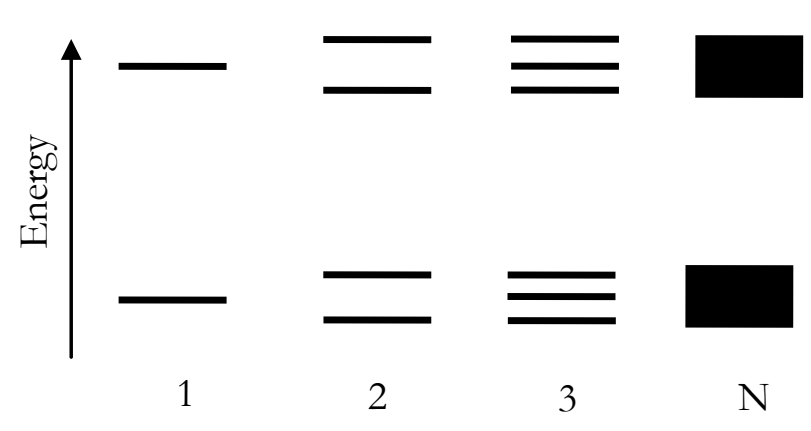

Figure 3.5. As many atoms come close together to form a solid, individual atomic orbitals of these atoms become nearly continuous. These allowed electron energies are separated by forbidden energy regions called band gaps. 
The bandwidth of an individual band is the difference between the highest energy and lowest energies in that band. Small bandwidths indicate localized orbital states while wide bandwidths indicate more delocalized bands. Most broadly, the band structure classifies the material as a conductor (metal), semiconductor, or insulator by a) the presence and width of a forbidden band gap region between the valance and conduction bands and b) the placement of the Fermi energy. The Fermi energy $E_{F}$ is the energy of the highest occupied molecular orbital (HOMO) at the ground state. Conductors do not possess a band gap since the Fermi energy lies in the middle of the band. Hence, unpaired electrons move freely in unoccupied excited states directly above the Fermi energy. Semiconductors and insulators both possess band gaps where $\mathrm{E}_{\mathrm{F}}$ is located. Semiconductors have relatively small band gaps that can be overcome by an electronic excitation, whether by thermal, electromagnetic, or other type of energy. Insulators have much larger band gaps such that an electronic excitation requires large energies and is much less likely to occur under average conditions. This material classification is shown in Figure 3.6.

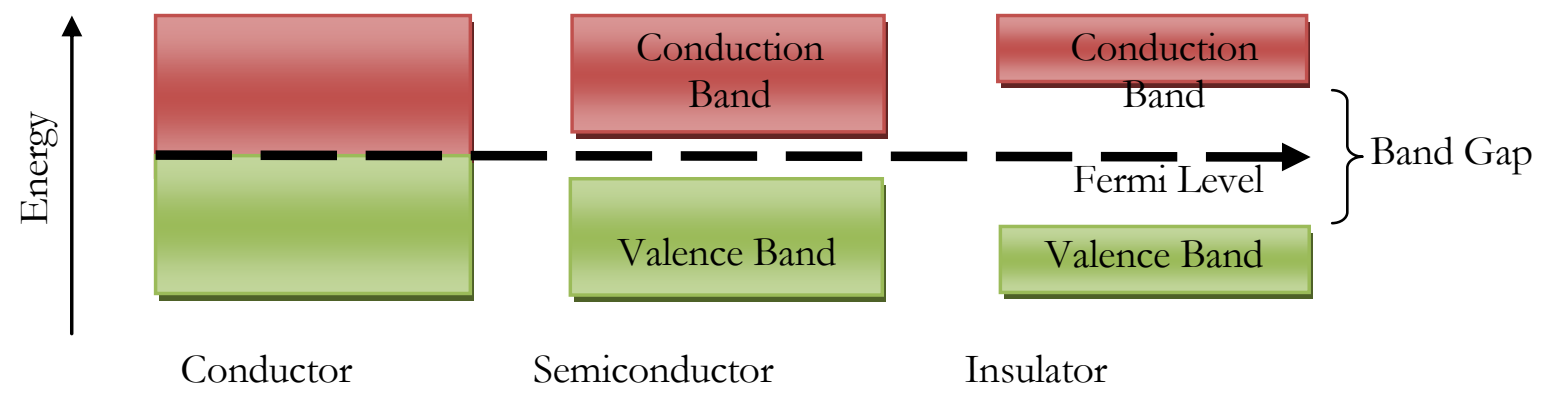

Figure 3.6. The size of the band gap between the conduction and valence bands classifies the type of material. The Fermi energy lies at the top of the valence band in all cases. The commonly referenced Fermi level or chemical potential lies within the band gap depending on the properties of charge carriers including their mobilities 


\subsubsection{Direct vs. Indirect Transitions}

Looking at where the VBM and CBM lie in $\boldsymbol{k}$-space can help us to understand if the material exhibits direct or indirect transitions ${ }^{35,37}$. A direct transition is a transition between the valance band and conduction band at the same point in $\boldsymbol{k}$ space, most commonly at the $\Gamma$-point. See Figure 3.7(a). Only photon energy is necessary for this kind of transition. An indirect transition is a transition between the valence band and conduction bands at different points in $\boldsymbol{k}$ space. See Figure 3.7(b). This transition requires both photon energy and phonon, or vibrational, energy. Phonons have an $\mathrm{E}(\omega)$ dispersion that typically has high momentum and low energy, allowing them to easily contribute to indirect transitions.

(a)

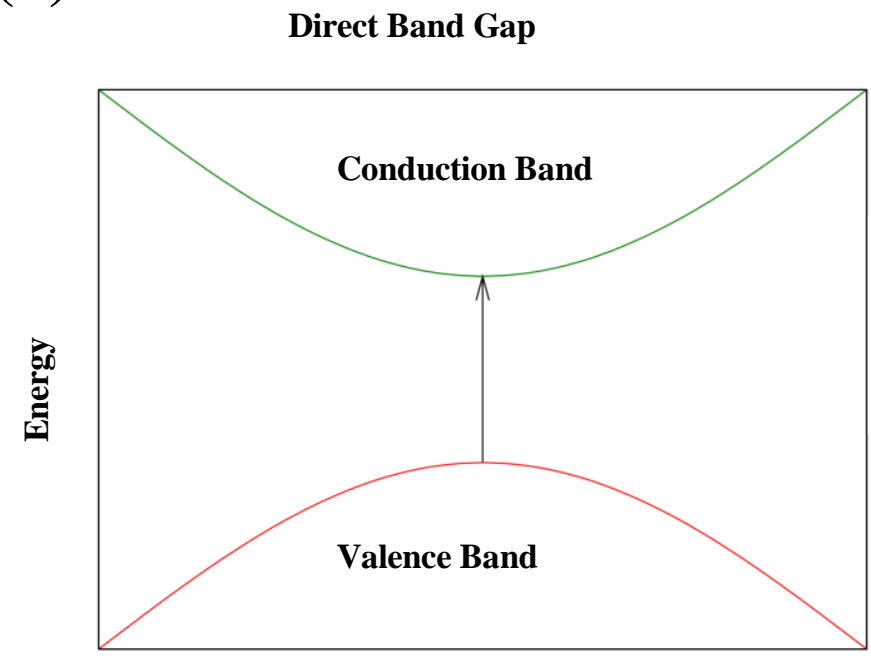

Momentum (b)

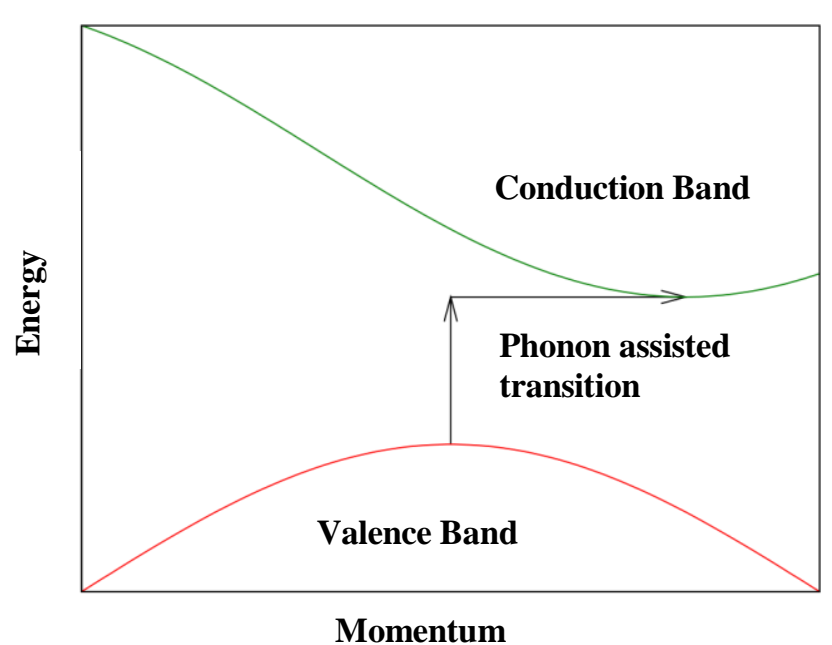

Figure 3.7. (a) A direct transition between orbitals occurs at the same point in momentum space needs only photon energy (b)A indirect transition occurs at different points in momentum space and requires both photon energy and phonon or vibrational energy. 
The probability of a transition $P$ occurring can be calculated by applying the electric dipole operator, $\widehat{\boldsymbol{d}}$ to the initial and final states of a transition

$$
P^{2}=\left\langle\Psi_{c}|\widehat{\boldsymbol{d}}| \Psi_{v}\right\rangle
$$

where the wavefunctions of the valence and conduction band are $\Psi_{\mathrm{v}}$ and $\Psi_{\mathrm{c}}$ respectively. In $\mathrm{CuGaO}_{2}$ and other $\mathrm{Cu}$-based delafossites, this probability of transition is zero due to the parity of the valence and conduction band being the same. For this reason, the fundamental transition is forbidden and the optically measured transition is of higher energy. 


\subsection{Electronic Density of States Calculation}

The Electronic Density of States (DOS) is a measure of the number of electrons per unit energy defined as

$\rho(E)=\frac{1}{N_{k}} \sum_{i, k} \delta\left(\varepsilon_{i, k}-E\right)$

Here $\varepsilon_{i, k}$ is the energy of the $\mathrm{i}^{\text {th }}$ electron at the $\mathrm{k}^{\text {th }} \mathrm{k}$-point in the system, $\mathrm{N}_{\mathrm{k}}$ is the number of electrons in the system, and $\delta$ is the delta function ${ }^{35}$. The band structure cannot be calculated for the B-site doped delafossites because they have broken symmetry. The DOS over the $\Gamma$-point for these structures is thus an invaluable measurement. These calculations allow us to observe any energy band gap in a material as in the electronic density of states as well as to get a feel for the number of orbitals at each energy level. Comparing DOS calculations will help us to understand the position and nature of defect states in the band. When states are added into the electronic structure through doping, they appear as new or altered peaks compared to the intrinsic structure. The DOS also allows for as estimation of the shifts in the VBM and CBM which are important for the PEC applications discussed. Furthermore, the partial DOS (PDOS) indicated which types of atoms are contributing to the modified states. The DOS can be viewed as a band structure turned on its side, the number, or density, of bands at each energy level in the band structure is proportional to the height of the peak in the DOS at that energy. In other words, the DOS of a particular band is inversely proportional to that band's slope with respect to $\mathbf{k}$. 


\subsection{W Calculations}

The localization of a state can be expressed by the quantity $\boldsymbol{W}$, which is derived from the Mulliken population. $\boldsymbol{W}$ is a quantization of the number of atoms at which an electron of a particular energy is likely to be found. ${ }^{38}$ A quantum entropy can be defined for a particular energy state $v$ from the Mulliken population as follows:

$$
S=-\sum p_{i}(v) \ln \left(p_{i}(v)\right)
$$

where $p_{i}(v)$ is the Mulliken population for a certain atom $i$ at energy state $v$, and is normalized so that $\sum_{i} p_{i}(v)=1$. Then the Boltzmann's equation is used to define $\boldsymbol{W}$ as the number of atoms the wavefunction $\phi(v)$.

$W=e^{S(v)}$

This measure of localization is particularly important for understanding the mobility of charge carriers in certain states. A low $\boldsymbol{W}$ indicates a highly localized state. This state may act as a trap state, or as a valence state unwilling to give up electrons which means decreased mobility for charge carriers. A high $\boldsymbol{W}$ means that the state is highly delocalized and has a propensity for electron transitions and an increased mobility. 


\section{Results}

All calculations were performed using the 2009 version of FIREBALL using a single numerical minimal basis set. The wavefunction cutoffs used were $3.4 \AA$ and $3.8 \AA$ for the oxygen 2-s and 2-p states; $5.3 \AA, 5.8 \AA$, and $4.7 \AA$ for the iron 4-s, 3-p, and 3- $d$ states; $5.1 \AA, 5.6 \AA$, and 4.6 $\AA$ for the copper 4-s, 3-p, and 3- $d$ states; and $4.8 \AA$ and $5.7 \AA$ for the gallium 4-s and 4- $d$ states.

For the electronic wavefunctions we also define a confinement potential that makes the wavefunction tail come to zero in a more smooth and exponential manner at the specified cutoff length. This confinement potential is defined as

$$
V_{c}=\frac{V_{0}}{C} e^{\frac{-r-r_{c}}{r_{0}-r}}
$$

where $\mathrm{r}_{\mathrm{c}}$ is the cutoff length, $V_{0}$ and $r_{0}$ are scaling factors, and $\mathrm{C}$ is normalization constant for the confinement potential. For the oxygen $2-d$ shell $\mathrm{V}_{0}=50$ and $\mathrm{r}_{0}=0.5$, for the iron 3- $p$ shell $\mathrm{V}_{0}=$ 100 and $\mathrm{r}_{0}=1.0$, for the copper 3-p shell $\mathrm{V}_{0}=50$ and $\mathrm{r}_{0}=0.5$, and for the gallium 4- $d$ shell $\mathrm{V}_{0}=$ 100 and $r_{0}=0$. Oxygen, gallium, and iron pseudopotentials were created using the Hamann model

39, while copper pseudopotentials were created using Troullier-Martins model ${ }^{40}$ which is more suitable for transition metals. The McWEDA expansion for evaluating multicenter exchangecorrelation interactions was utilized throughout this work and is proven to be effective for other oxide materials ${ }^{4 \mathrm{~g}, 41}$. 


\subsection{Electronic Properties of Bulk $\mathrm{CuGaO}_{2}$ and $\mathrm{CuFeO}_{2}$}

The electronic band structures were calculated and plotted for both $\mathrm{CuGaO}_{2}$ and $\mathrm{CuFeO}_{2}$ from unit cells constructed by the scheme described in Section 3.1.1. Figures 4.1 and 4.2 show the electronic band structure for the bulk structures. These calculations are derived from the primitive unit cell. For $\mathrm{CuGaO}_{2}$ direct $\Gamma$ transitions are seen at approximately $1.81 \mathrm{eV}$ and $4.68 \mathrm{eV}$ from the valance band to the first $(\mathrm{X})$ and second $(\mathrm{Y})$ conduction bands, respectively. Also observed are indirect transitions from $\mathrm{L}$ to $\Gamma$ of about $0.91 \mathrm{eV}$ and $3.8 \mathrm{eV}$ from the valence band to the first $(\mathrm{X})$ and second (Y) conduction bands, respectively. There is also a direct $\mathrm{Z}$ transition of around 4.0 eV. For $\mathrm{CuFeO}_{2}$, calculations show three intermediate states. This material exhibits a direct $\mathrm{L}$ transition at $1.64 \mathrm{eV}$ to the conduction band, and indirect transition from $\mathrm{L}$ to $\Gamma$ at $1.42 \mathrm{eV}$. There is also a direct $\Gamma$ transition at $2.41 \mathrm{eV}$. These results are in good agreement with previous work. ${ }^{42}$

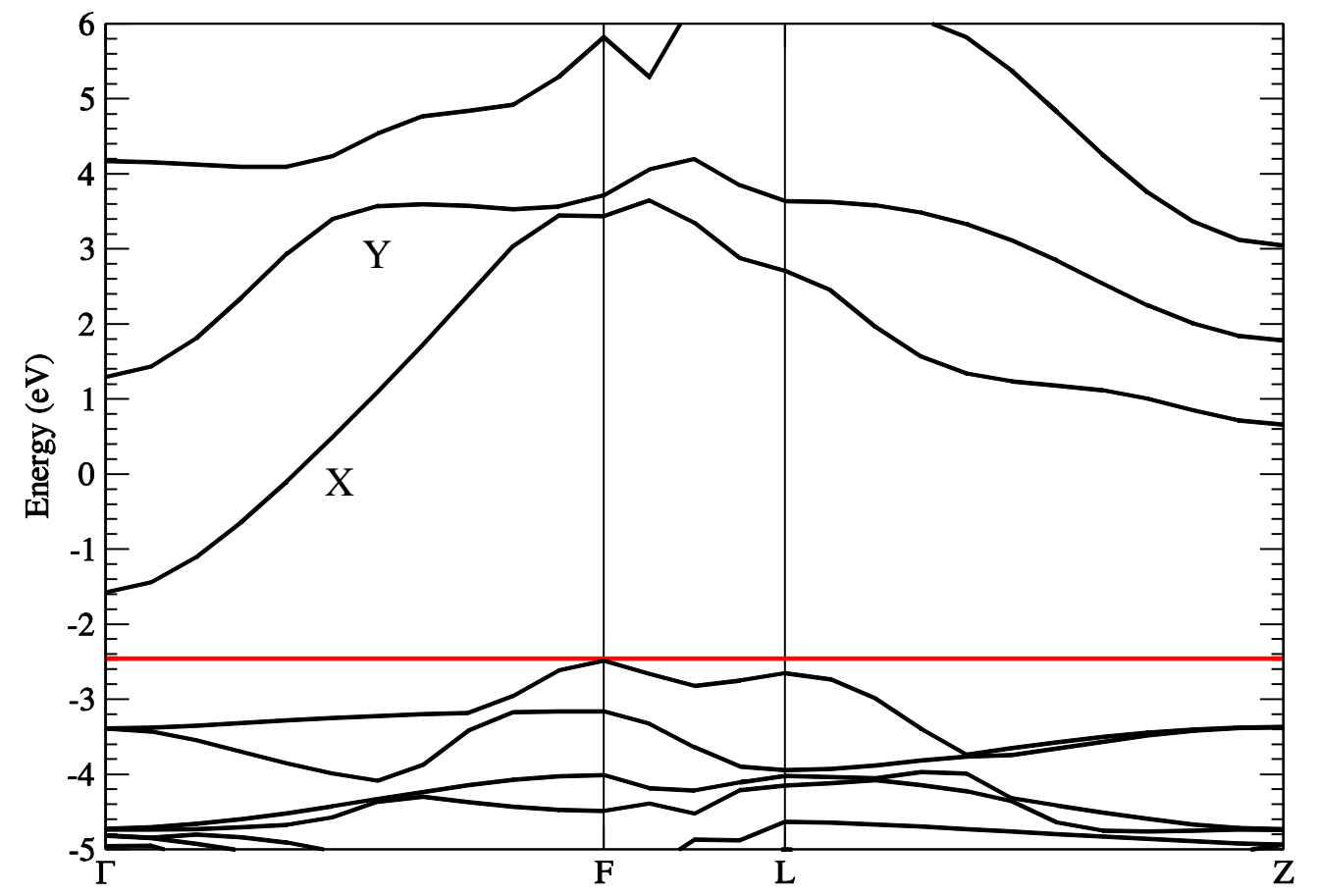

Figure 4.1. Electronic band structure for $\mathrm{CuGaO}_{2}$ bulk structure where the Fermi level is denoted by the red horizontal line. 


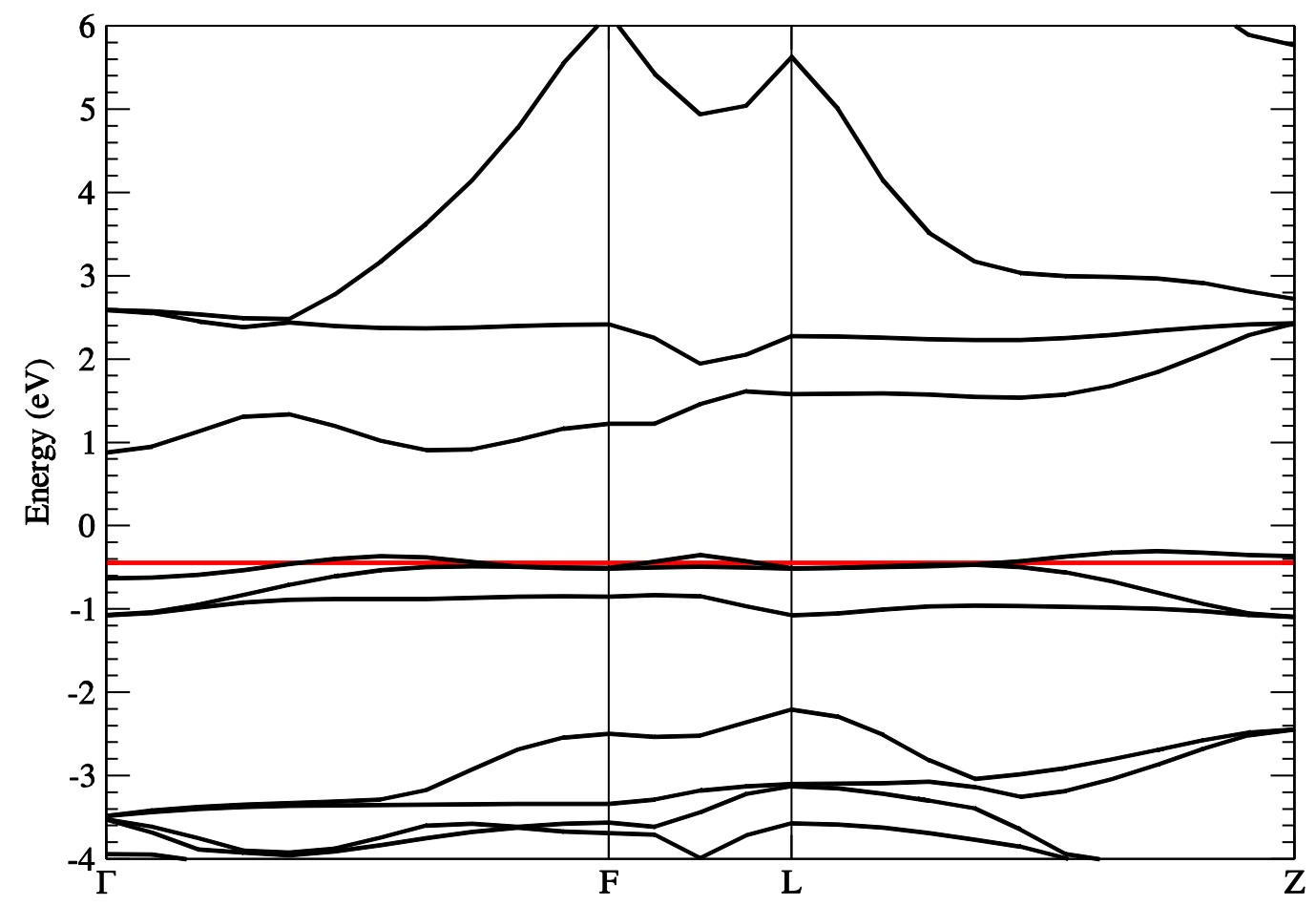

Figure 4.2. Electronic band structure for $\mathrm{CuFeO}_{2}$ bulk structure where the Fermi level is denoted by the red horizontal line.

From supercells of 192 atoms created by the method described in Section 3.1.2, we calculate the DOS, PDOS, and $\boldsymbol{W}$ plots of bulk $\mathrm{CuGaO}_{2}$ and $\mathrm{CuFeO}_{2}$ as shown in Figures 4.3 to 4.6. These calculations will act as direct references to understand the changes in the electronic structure due to B-site doping. 


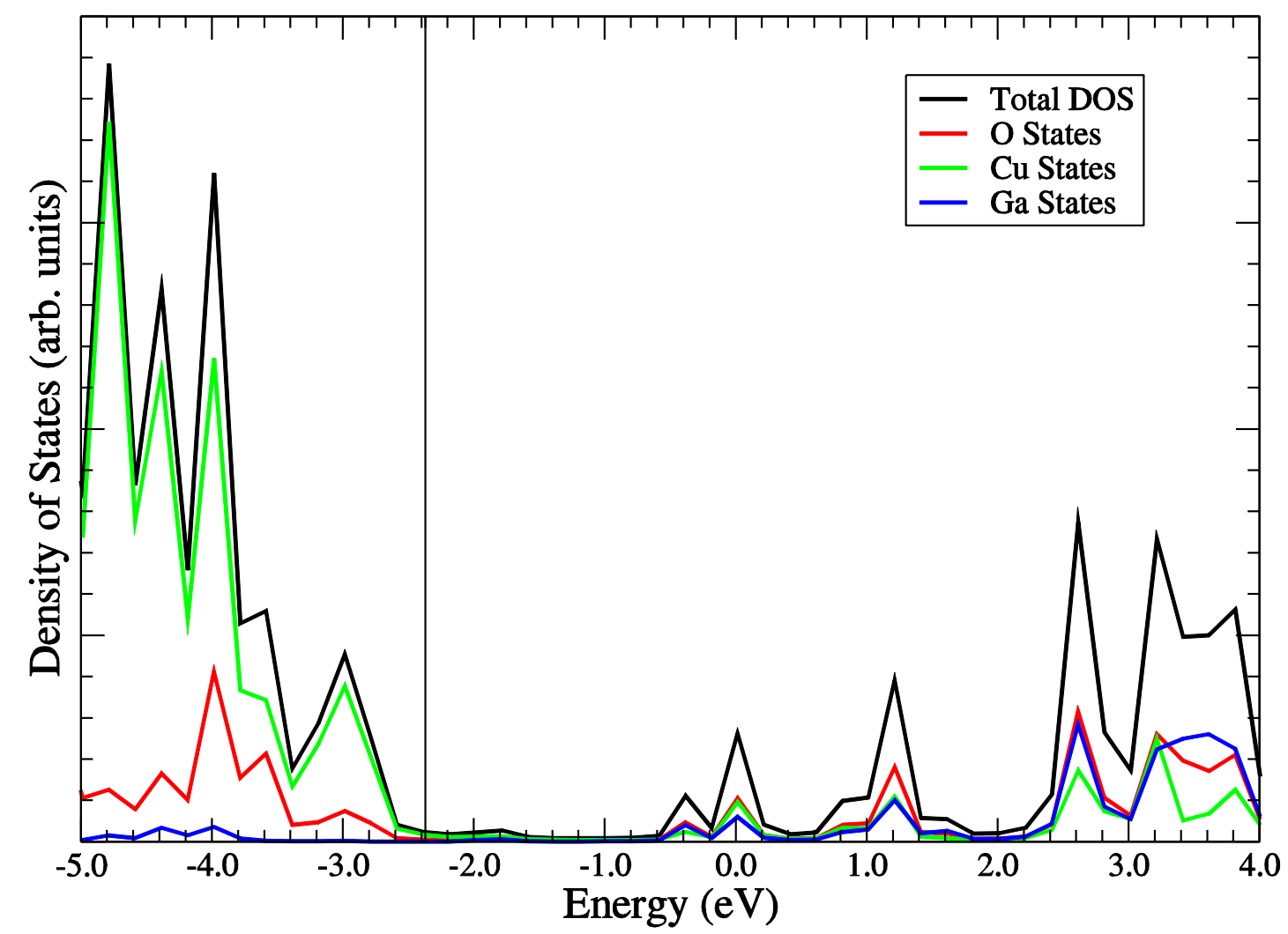

Figure 4.3. Electronic density of states for $\mathrm{CuGaO}_{2}$ bulk structure where the Fermi level is denoted by the black vertical line.

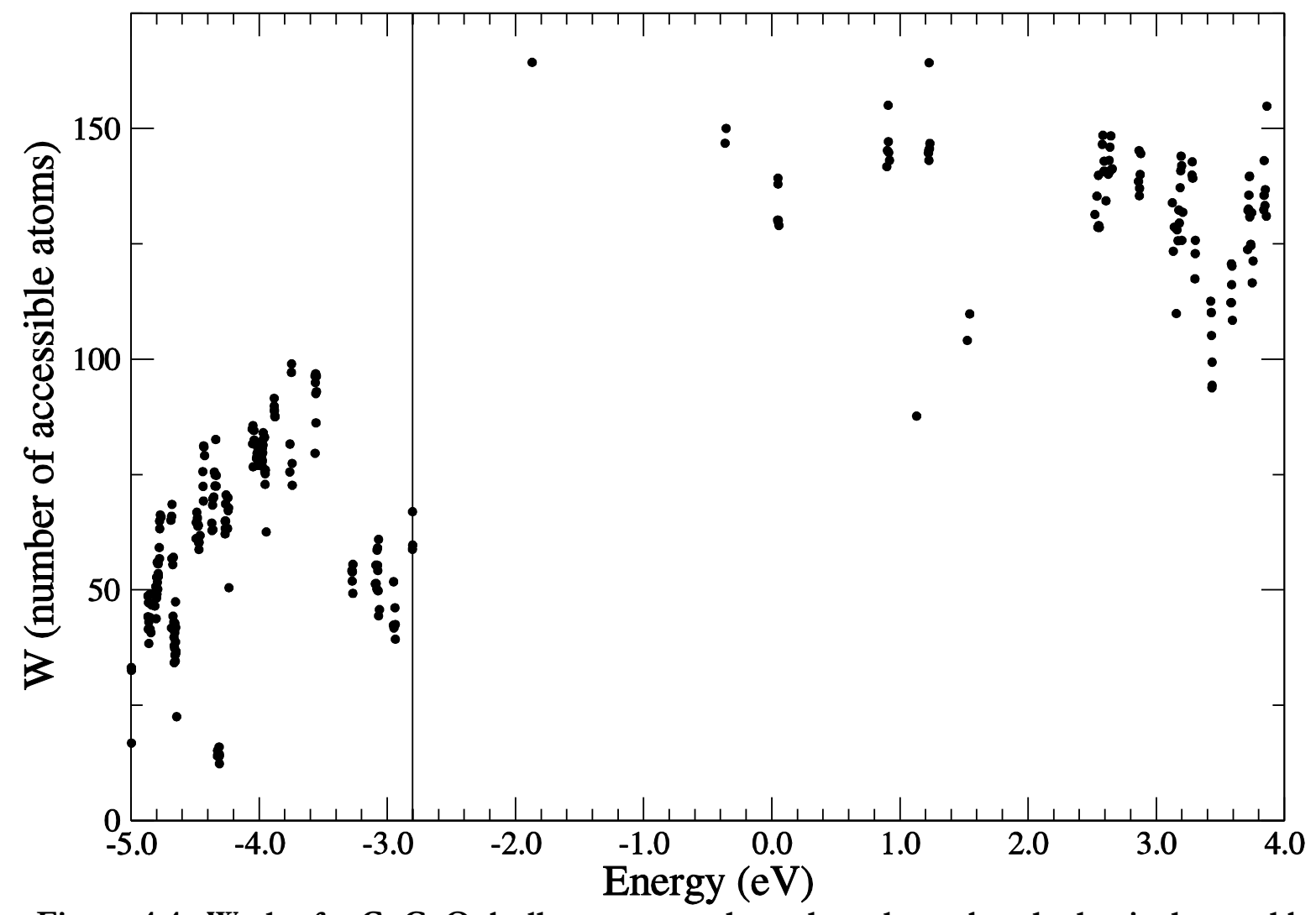

Figure 4.4. $\mathrm{W}$ plot for $\mathrm{CuGaO}_{2}$ bulk structure where the valence band edge is denoted by the black vertical line. 


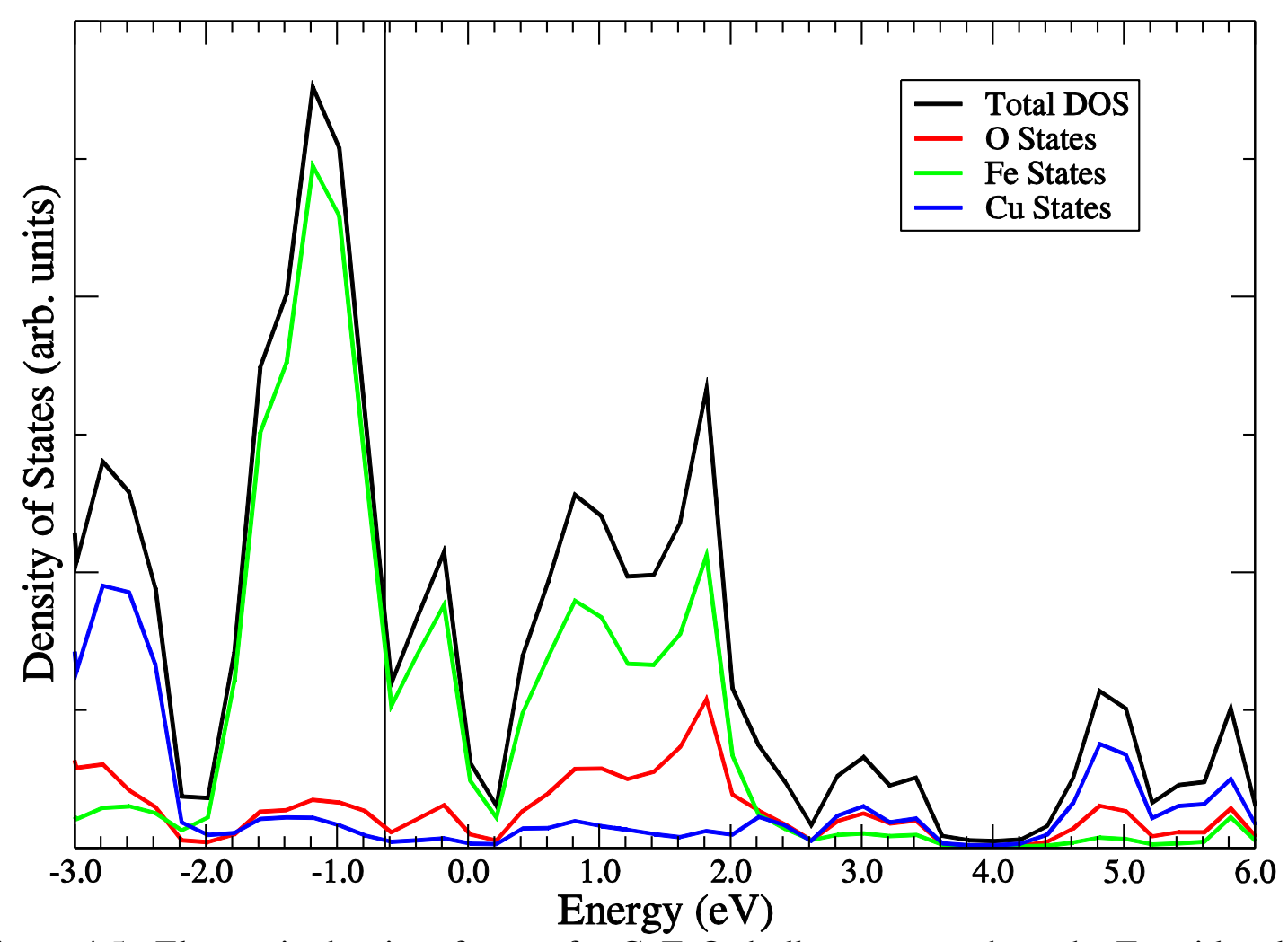

Figure 4.5. Electronic density of states for $\mathrm{CuFeO}_{2}$ bulk structure where the Fermi level is denoted by the black vertical line.

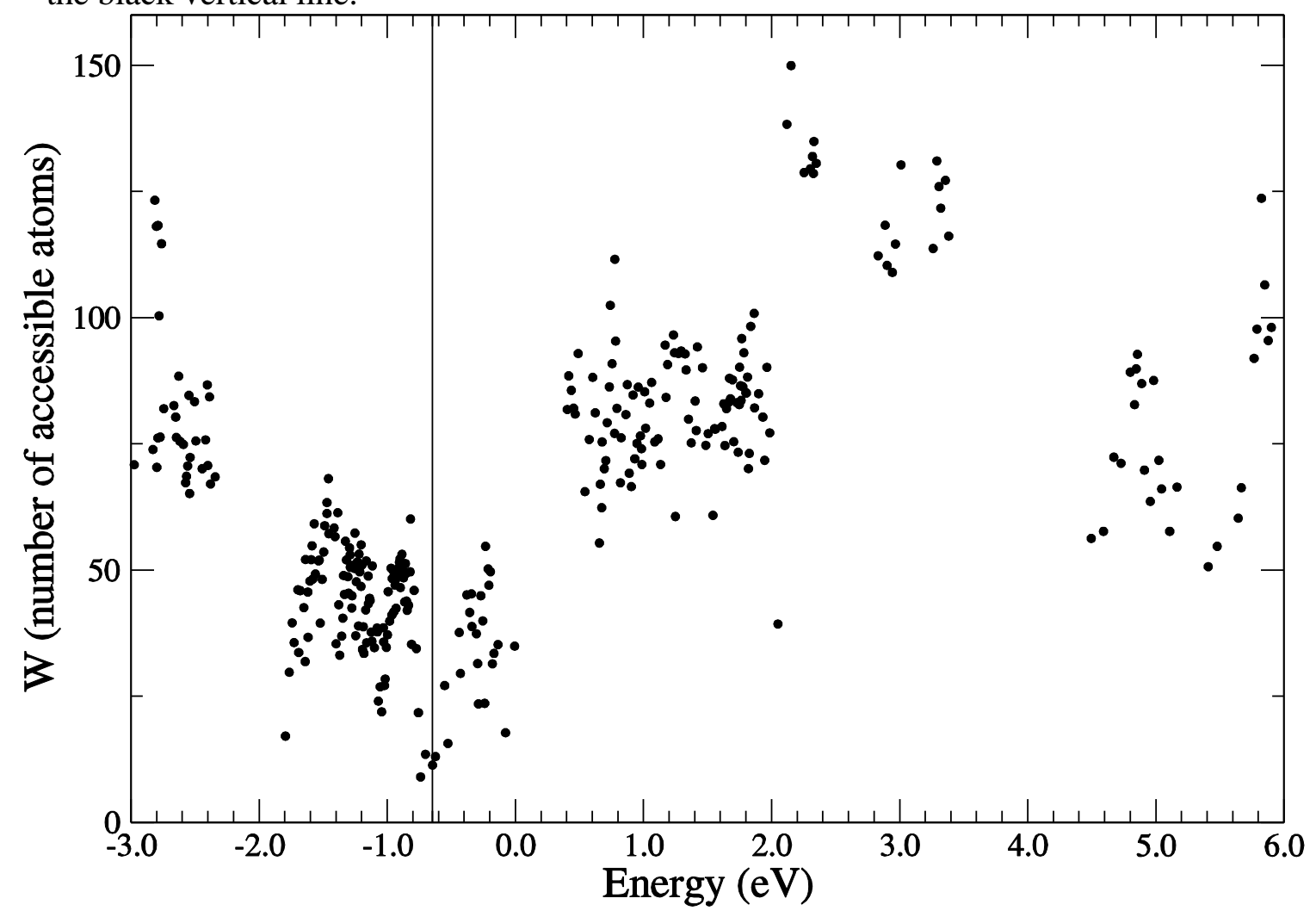

Figure 4.6. W plot for $\mathrm{CuFeO}_{2}$ bulk structure where the valence band edge is denoted by the black vertical line. 
4.2 Electronic Properties of $\mathrm{CuGa}_{1-\mathrm{x}} \mathrm{Fe}_{\mathrm{x}} \mathrm{O}_{2}$ where $\mathrm{x}=0.05,0.10,0.15$, and 0.20

The first calculations that were performed on doped delafossites $\mathrm{CuGa}_{1-\mathrm{x}} \mathrm{Fe}_{\mathrm{x}} \mathrm{O}_{2}$ were performed on supercells created with the experimental lattice constants described in Section 3.1.1 and extended in the lattice vector directions by 4,4 , and 4 creating a long skinny supercell structure that mimics the shape of the unit cell. These were performed on structures where $\mathrm{x}=0.05,0.10$, 0.15 , and 0.20 in conjunction with the experimental portion of this research at the Department of Energy National Energy Technology Laboratory in Pittsburgh, PA. Those results are shown along with experimental results in the Journal of Physical Chemistry C. ${ }^{34}$ The following results are improvements upon those initial calculations performed on supercells of 192 atoms created by the procedure described in Section 3.1.2. Figures 4.7 to 4.14 show the DOS, PDOS and $\boldsymbol{W}$ results for one doped delafossite structure at each doping percentage. The vertical line in the DOS plots shows the Fermi level as calculated by FIREBALL. The vertical line in the $\mathrm{W}$ plots shows the ground state valence band edges as calculated by the number of electrons in the structure and the energy eigenvalues that describe the energies of each molecular orbital. The exact values for the Fermi level and valence band edge of each structure are compiled in Table 4.1 below.

\begin{tabular}{|c|c|c|c|}
\hline Structure & Fermi Level (eV) & $\begin{array}{c}\text { Number of Valence } \\
\text { Electrons }\end{array}$ & Valence band edge (eV) \\
\hline $\mathrm{CuGaO}_{2}$ & -2.3689 & 1248 & -2.80252 \\
\hline $\mathrm{CuGa}_{0.95} \mathrm{Fe}_{0.05} \mathrm{O}_{2}$ & -1.8977 & 1258 & -2.00283 \\
\hline $\mathrm{CuGa}_{0.90} \mathrm{Fe}_{0.10} \mathrm{O}_{2}$ & -1.8743 & 1273 & -1.89644 \\
\hline $\mathrm{CuGa}_{0.85} \mathrm{Fe}_{0.15} \mathrm{O}_{2}$ & -1.8008 & 1283 & -1.81674 \\
\hline $\mathrm{CuGa}_{0.80} \mathrm{Fe}_{0.20} \mathrm{O}_{2}$ & -1.5861 & 1298 & -1.59161 \\
\hline $\mathrm{CuFeO}_{2}$ & -0.6330 & 1488 & -0.64644 \\
\hline
\end{tabular}

Table 4.1. Fermi level, total number of contributing electrons and valence band edge for each of the $\mathrm{CuGa}_{1-\mathrm{x}} \mathrm{Fe}_{\mathrm{x}} \mathrm{O}_{2}$ structures where $\mathrm{x}=0.05,0.10,0.15$, and 0.20 . 


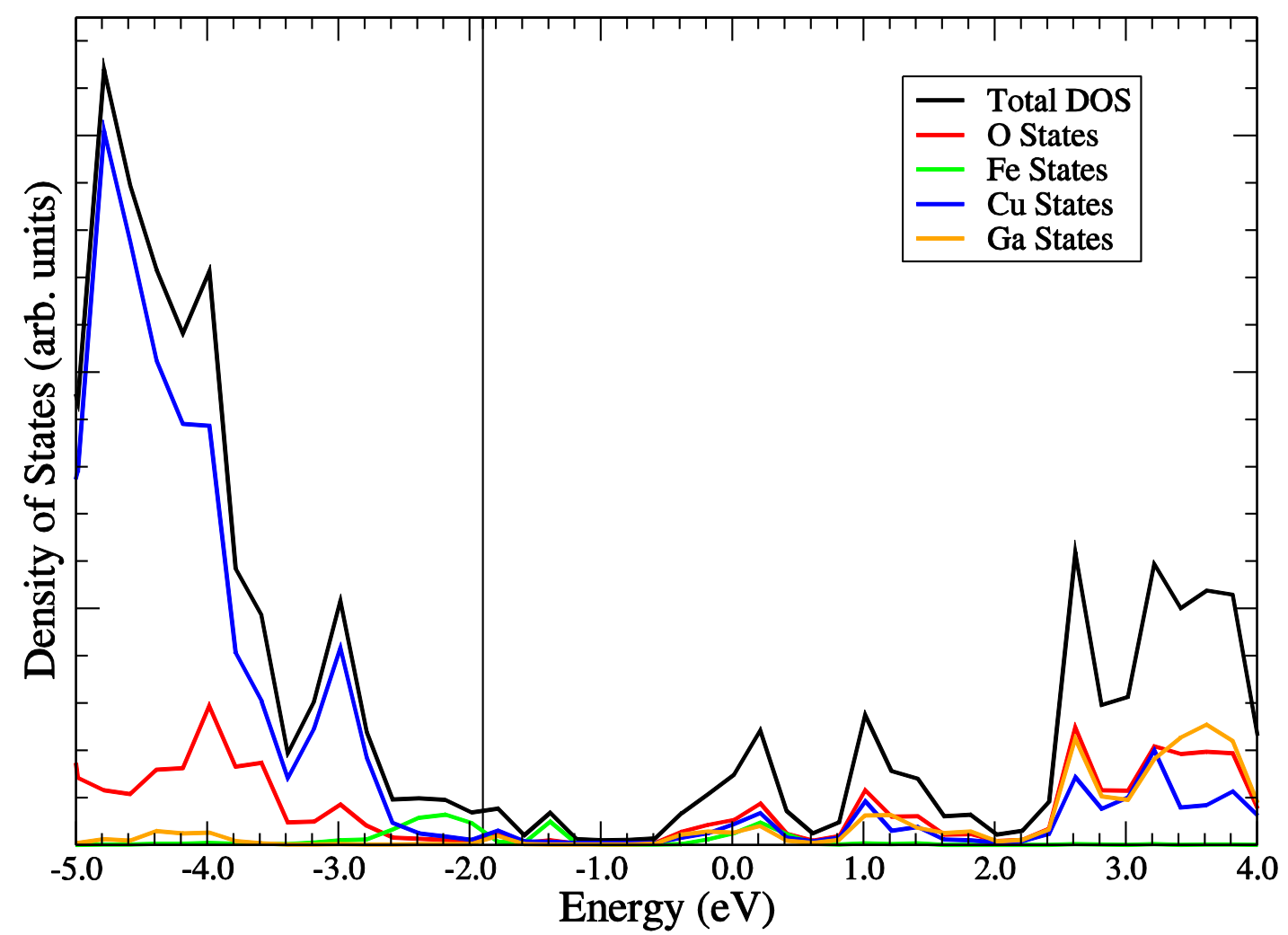

Figure 4.7. Electronic density of states for $\mathrm{CuGa}_{0.95} \mathrm{Fe}_{0.05} \mathrm{O}_{2}$ bulk structure where the Fermi level is denoted by the black vertical line.

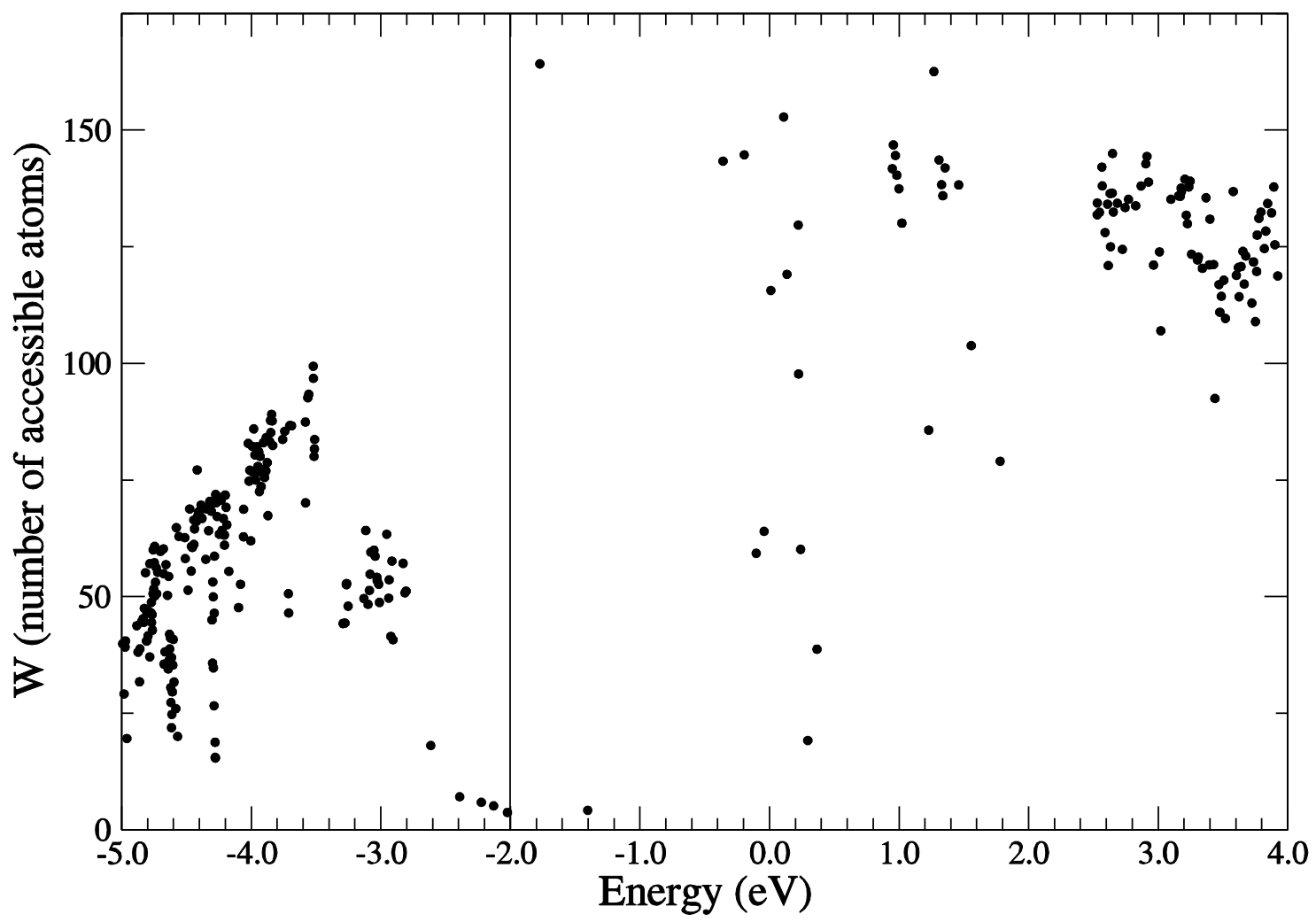

Figure 4.8. W plot for $\mathrm{CuGa}_{0.95} \mathrm{Fe}_{0.05} \mathrm{O}_{2}$ bulk structure where the valence band edge is denoted bv the black vertical line. 


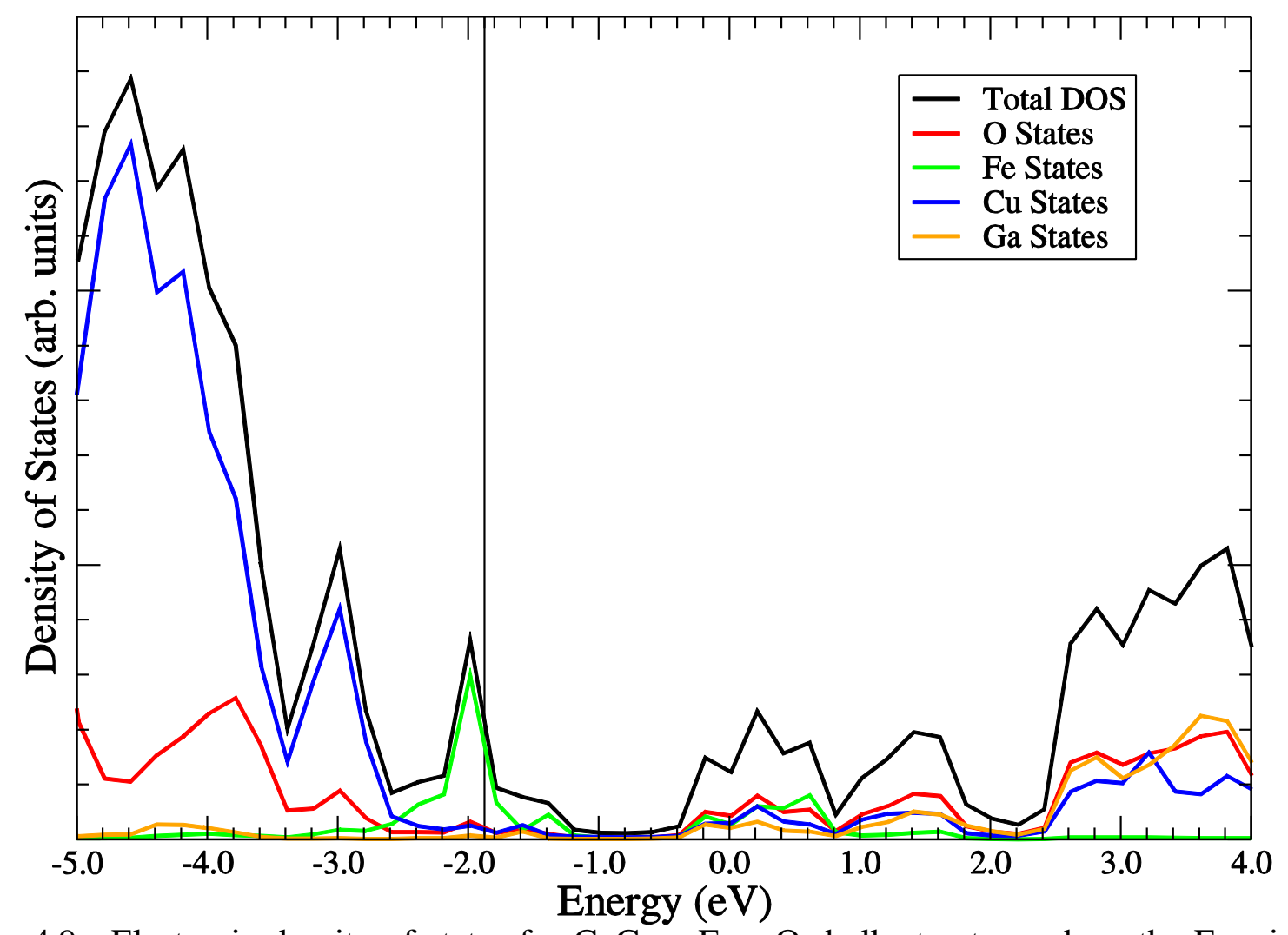

Figure 4.9. Electronic density of states for $\mathrm{CuGa}_{0.90} \mathrm{Fe}_{0.10} \mathrm{O}_{2}$ bulk structure where the Fermi level is denoted by the black vertical line.

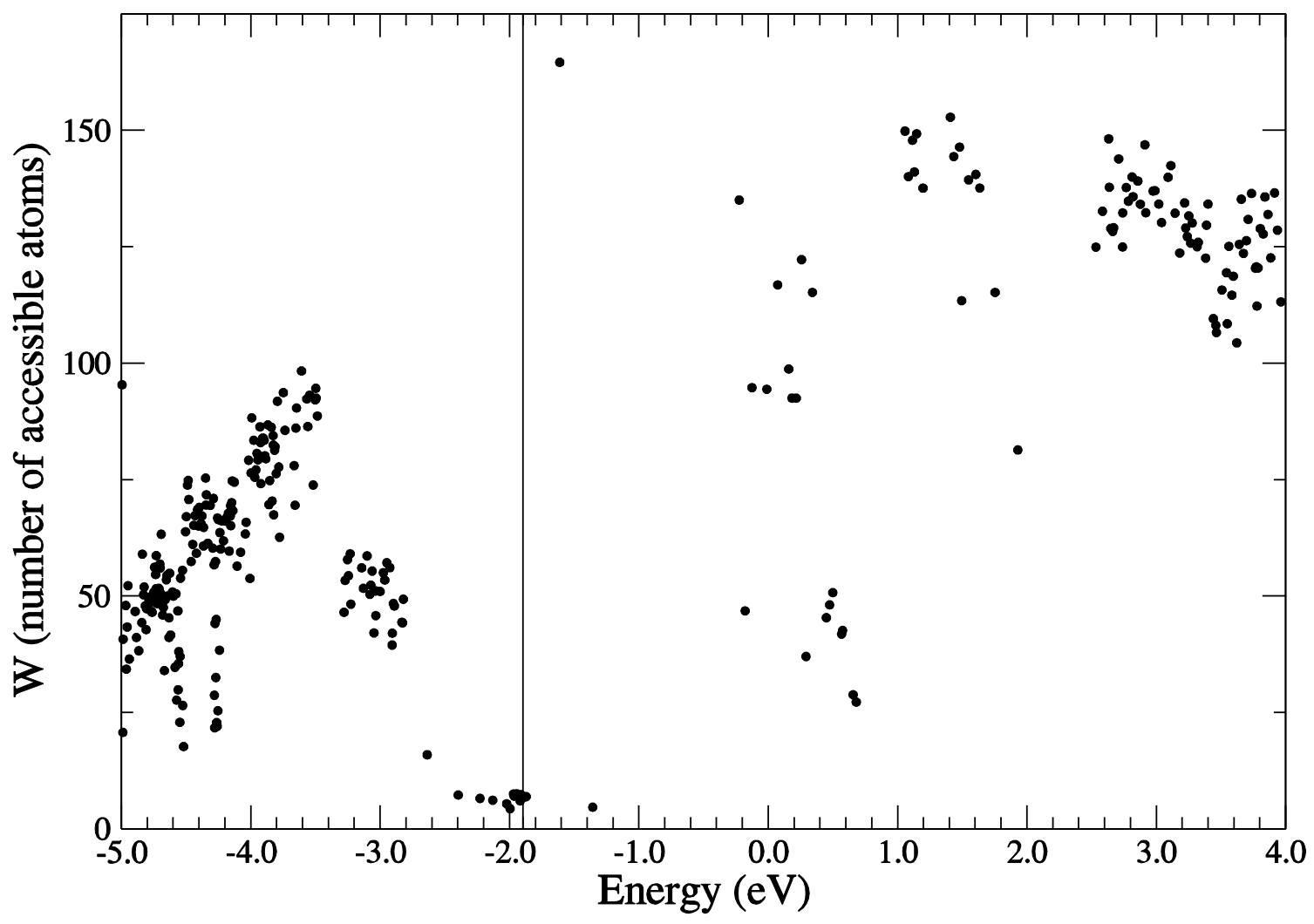

Figure 4.10. W plot for $\mathrm{CuGa}_{0.90} \mathrm{Fe}_{0.10} \mathrm{O}_{2}$ bulk structure where the valence band edge is denoted by the black vertical line. 


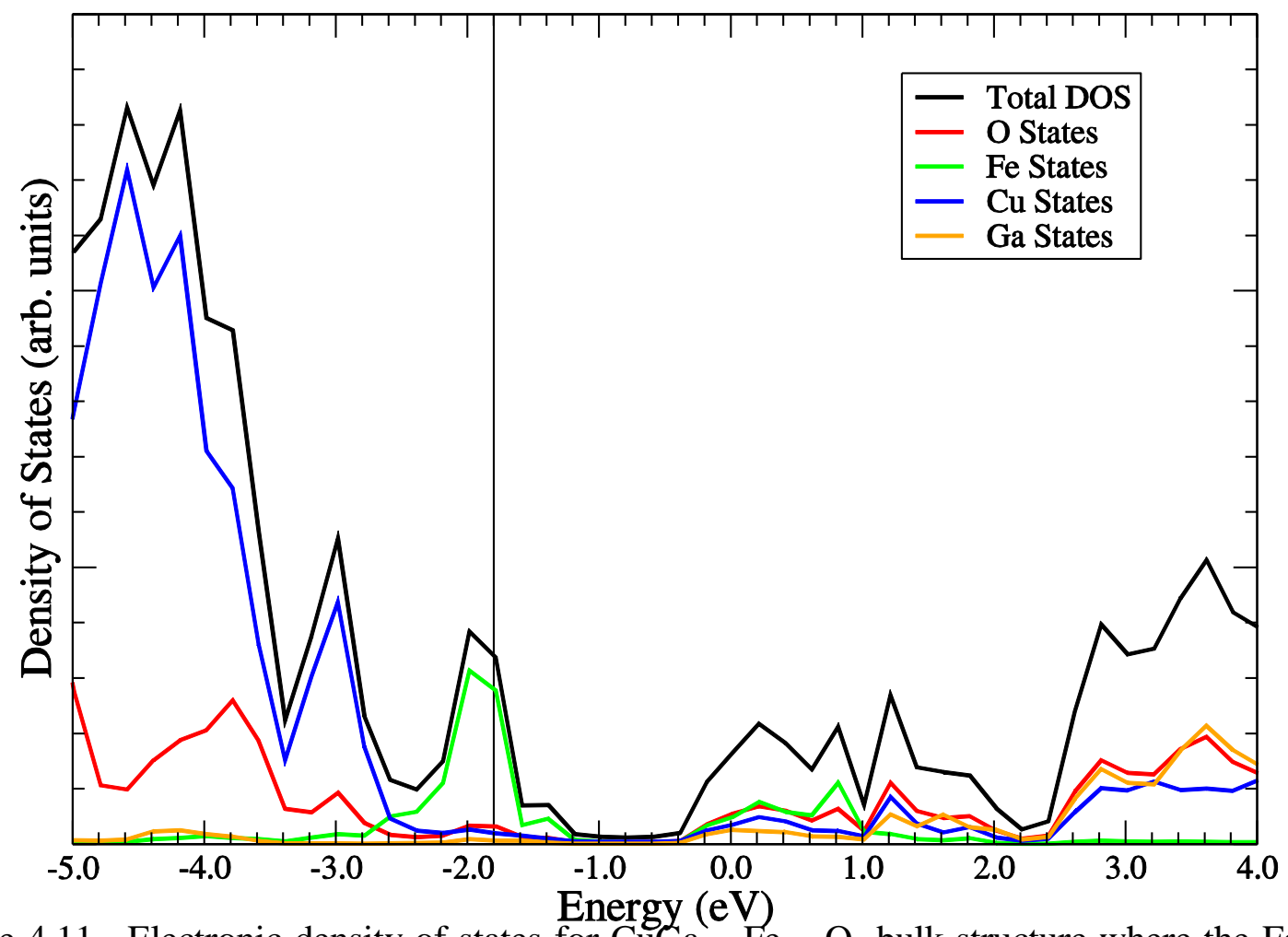

Figure 4.11. Electronic density of states for $\mathrm{CuGa}_{0.85} \mathrm{Fe}_{0.15} \mathrm{O}_{2}$ bulk structure where the Fermi level is denoted by the black vertical line.

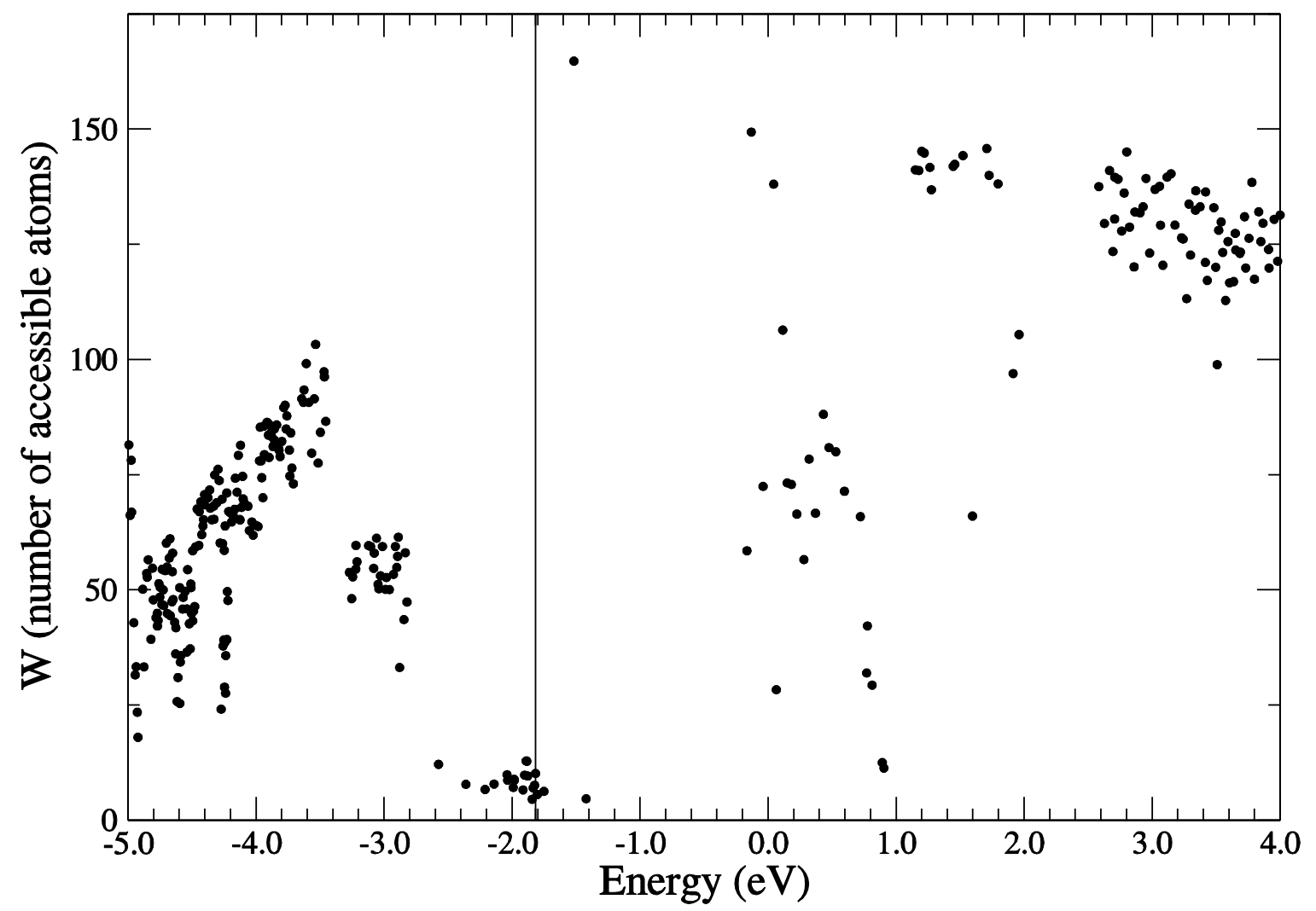

Figure 4.12. W plot for $\mathrm{CuGa}_{0.85} \mathrm{Fe}_{0.15} \mathrm{O}_{2}$ bulk structure where the valence band edge is denoted by the black vertical line. 


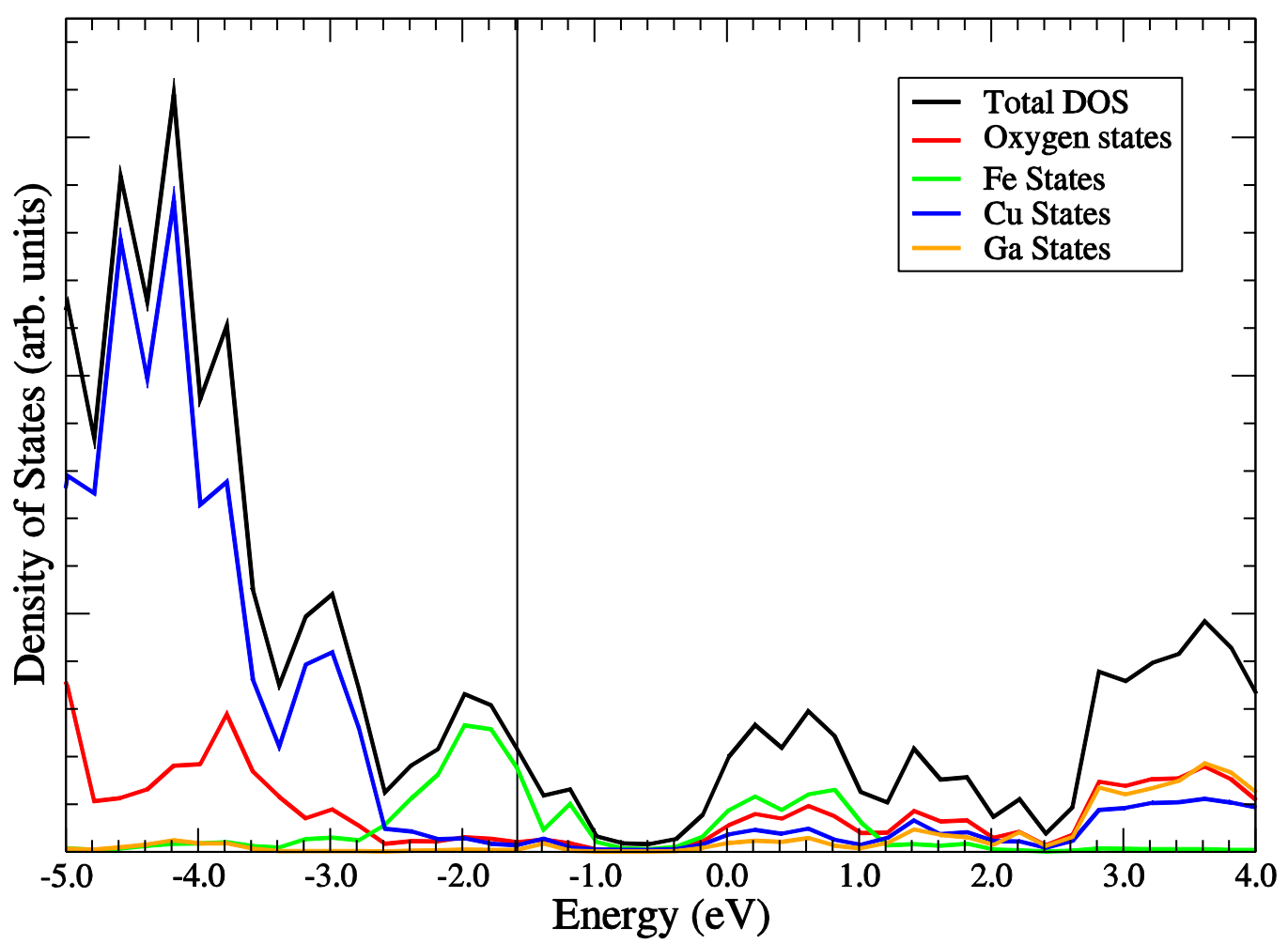

Figure 4.13. Electronic density of states for $\mathrm{CuGa}_{0.80} \mathrm{Fe}_{0.20} \mathrm{O}_{2}$ bulk structure where the Fermi level is denoted by the black vertical line.

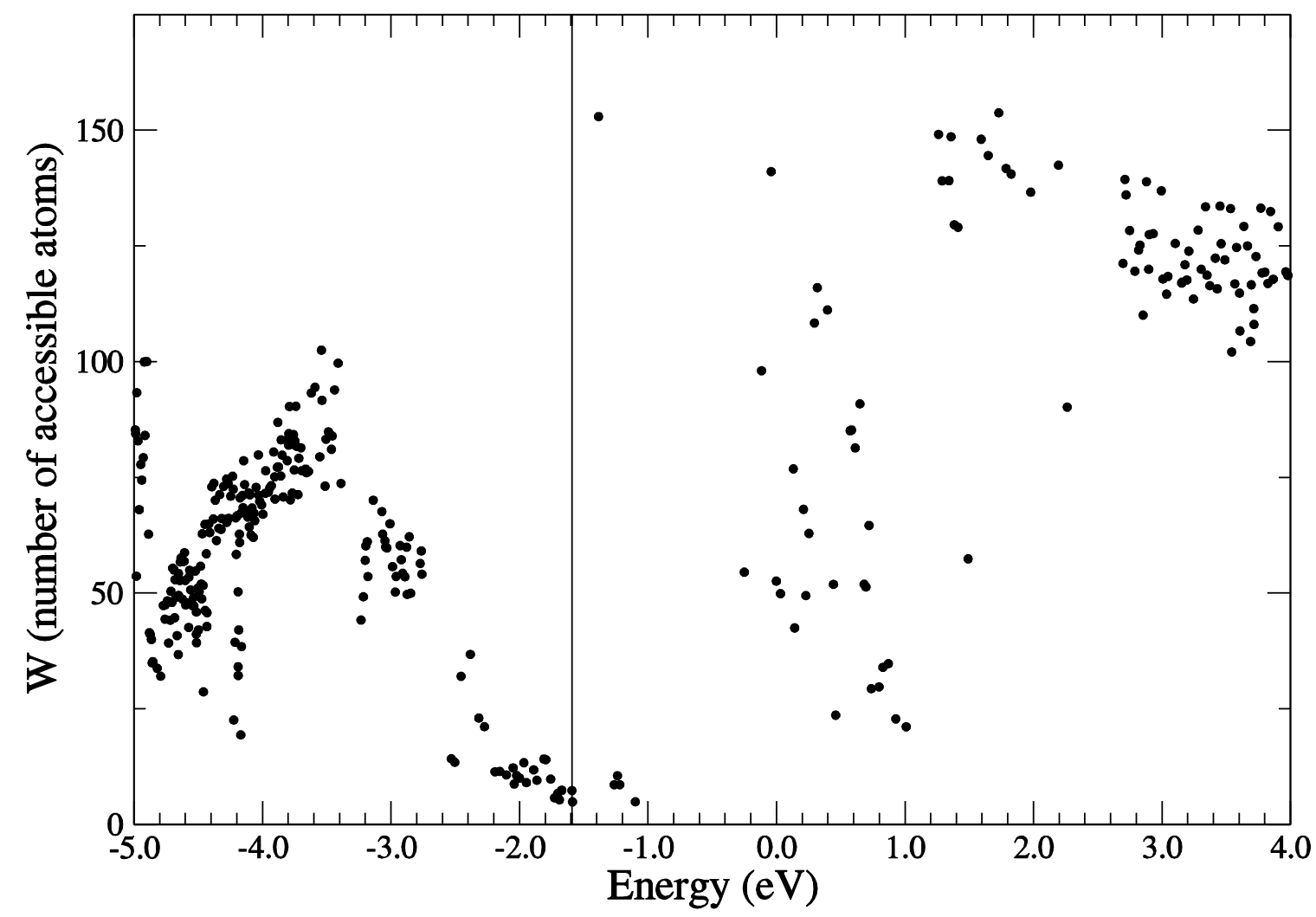

Figure 4.14. $\mathrm{W}$ plot for $\mathrm{CuGa}_{0.80} \mathrm{Fe}_{0.20} \mathrm{O}_{2}$ bulk structure where the valence band edge is denoted by the black vertical line. 


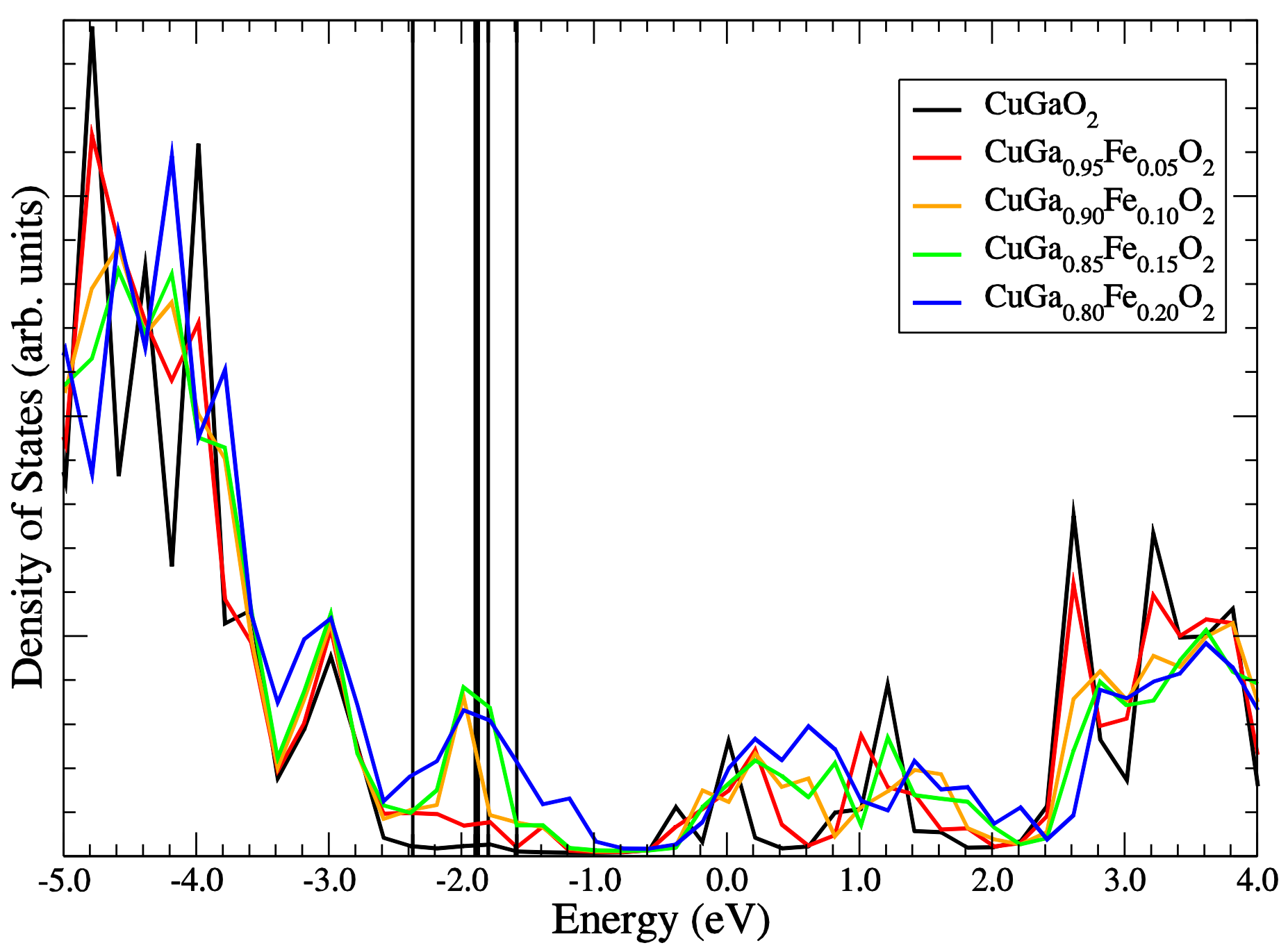

Figure 4.15. DOS plots for structures with doping percentages $0.05,0.10,0.15$, and 0.20 overlaid with the DOS plot of the intrinsic $\mathrm{CuGaO}_{2}$ structure with vertical lines denoting the Fermi levels increasing in energy with increasing doping percentage.

Examining at Figure 4.15, the molecular orbitals of the added Fe atoms contribute to features in the band gap around $-2 \mathrm{eV}$. The act of doping the structure also adds features between $0 \mathrm{eV}$ and $2 \mathrm{eV}$ by broadening the states in these areas. The PDOS of these doped structure show that the features near $-2 \mathrm{eV}$ are due to Fe states while the features between $0 \mathrm{eV}$ and $2 \mathrm{eV}$ are due to mostly $\mathrm{O}$ states, and thus are an effect from changing the structure by doping, rather than from the added atoms themselves. We attribute these states to the breaking of symmetry in the system and the strain therefore added on the $\mathrm{O}$ atoms. This break in symmetry is the reason attributed to 
the increase in visible light absorption for doped structures observed experimentally. ${ }^{34}$ It was found that though visible light absorption is increased, doping the structure past 5 percent iron adds no measureable improvement in visible light absorption. To understand why this is, we continued research to observe small doping percentages leading up to 5 percent. Also, regardless of the increase in visible light absorption $\mathrm{CO}_{2}$ reduction still requires $\mathrm{UV}$ light for all doping percentages. 
4.3 Electronic Properties of $\mathrm{CuGa}_{1-\mathrm{x}} \mathrm{Fe}_{\mathrm{x}} \mathrm{O}_{2}$ where $\mathrm{x}=0.01,0.02,0.03$, and 0.04

To understand the saturation of increased visible light absorption at 5 percent iron doping of $\mathrm{CuGaO}_{2}$, we explore the electronic properties of $\mathrm{CuGa}_{1-\mathrm{x}} \mathrm{Fe}_{\mathrm{x}} \mathrm{O}_{2}$ where $\mathrm{x}=0.01,0.02,0.03$, and 0.04. Calculations were performed on supercells of 432 atoms created by the method described in Section 3.1.2. The DOS, PDOS, and W plots for one structure of each doping percentage are shown in Figures 4.16 to 4.23. The vertical line in the DOS plots shows the Fermi level as calculated by FireBALL. The vertical line in the $\mathrm{W}$ plots shows the ground state valence band edges as calculated by the number of electrons in the structure and the energy eigenvalues which describe the energies of each molecular orbital. The DOS for all of these minimally doped structures are overlaid in Figure 4.24. The exact values for the Fermi level and valence band edge of each structure are compiled in Table 4.2 below.

\begin{tabular}{|c|c|c|c|}
\hline Structure & Fermi Level (eV) & $\begin{array}{c}\text { Number of Valence } \\
\text { Electrons }\end{array}$ & Valence band edge (eV) \\
\hline $\mathrm{CuGaO}_{2}$ & -2.3689 & 1248 & -2.80252 \\
\hline $\mathrm{CuGa}_{0.99} \mathrm{Fe}_{0.01} \mathrm{O}_{2}$ & -2.03303 & 2813 & -2.06339 \\
\hline $\mathrm{CuGa}_{0.98} \mathrm{Fe}_{0.02} \mathrm{O}_{2}$ & -2.02217 & 2818 & -2.05542 \\
\hline $\mathrm{CuGa}_{0.97} \mathrm{Fe}_{0.03} \mathrm{O}_{2}$ & -2.01206 & 2823 & -2.04192 \\
\hline $\mathrm{CuGa}_{0.96} \mathrm{Fe}_{0.04} \mathrm{O}_{2}$ & -1.97218 & 2828 & -1.99540 \\
\hline $\mathrm{CuFeO}_{2}$ & -0.6330 & 1488 & -0.64644 \\
\hline
\end{tabular}

Table 4.2. Fermi level, total number of contributing electrons and valence band edge for each of the $\mathrm{CuGa}_{1-\mathrm{x}} \mathrm{Fe}_{\mathrm{x}} \mathrm{O}_{2}$ structures where $\mathrm{x}=0.010 .02,0.03$, and 0.04 . 


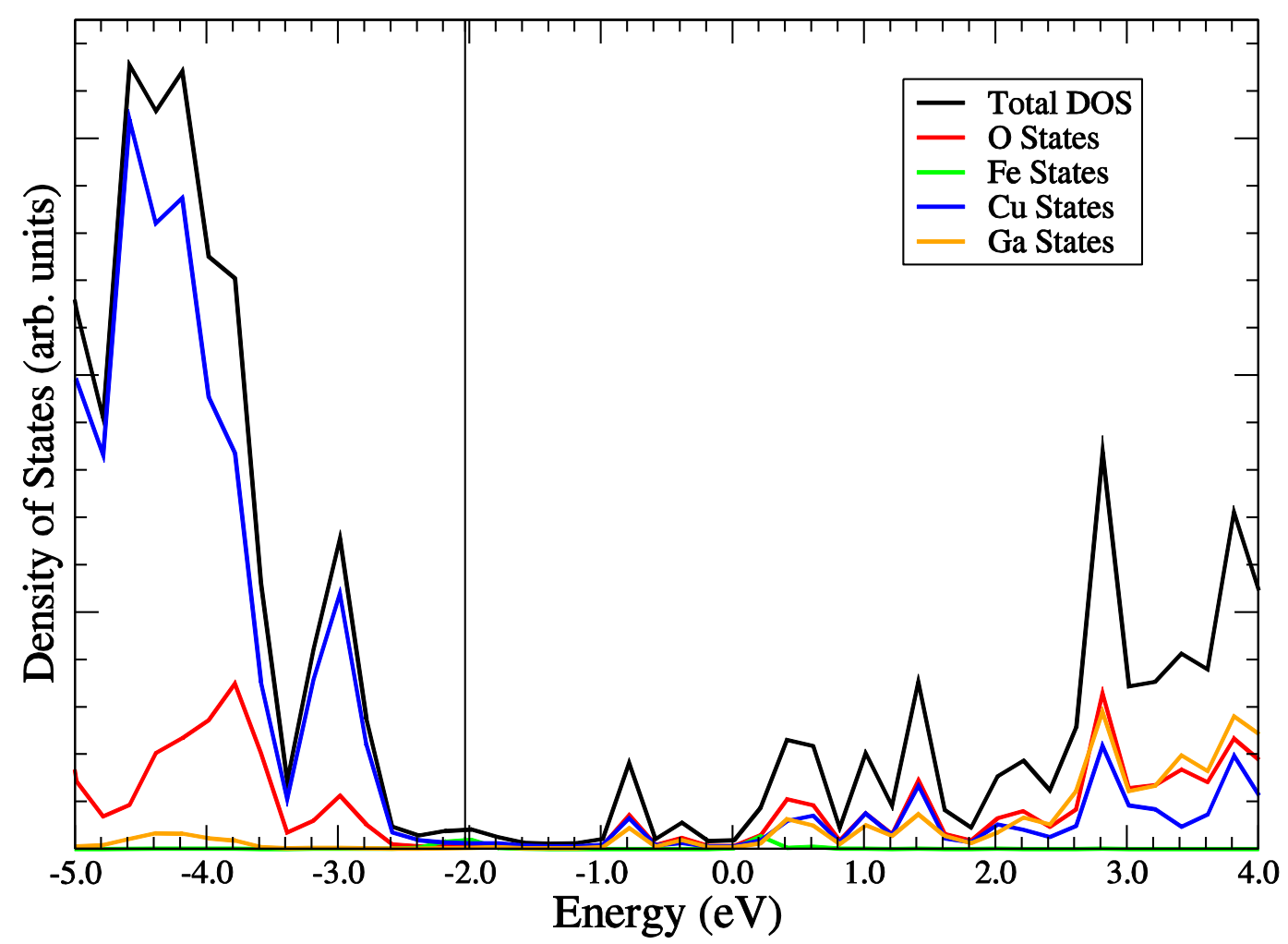

Figure 4.16. Electronic density of states for $\mathrm{CuGa}_{0.99} \mathrm{Fe}_{0.01} \mathrm{O}_{2}$ bulk structure where the Fermi level is denoted by the black vertical line.

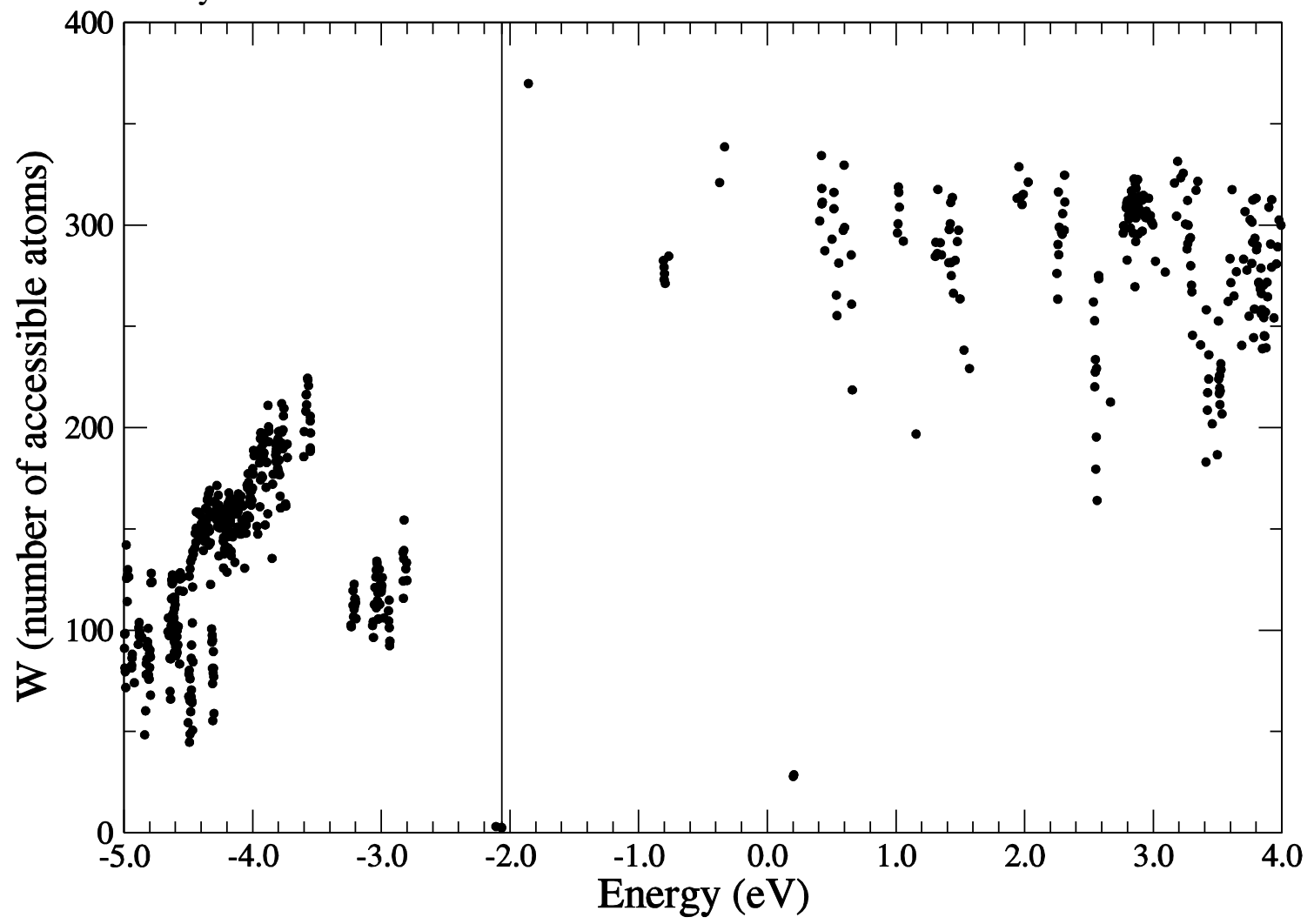

Figure 4.17. $\mathrm{W}$ plot for $\mathrm{CuGa}_{0.99} \mathrm{Fe}_{0.01} \mathrm{O}_{2}$ bulk structure where the valence band edge is denoted by the black vertical line. 


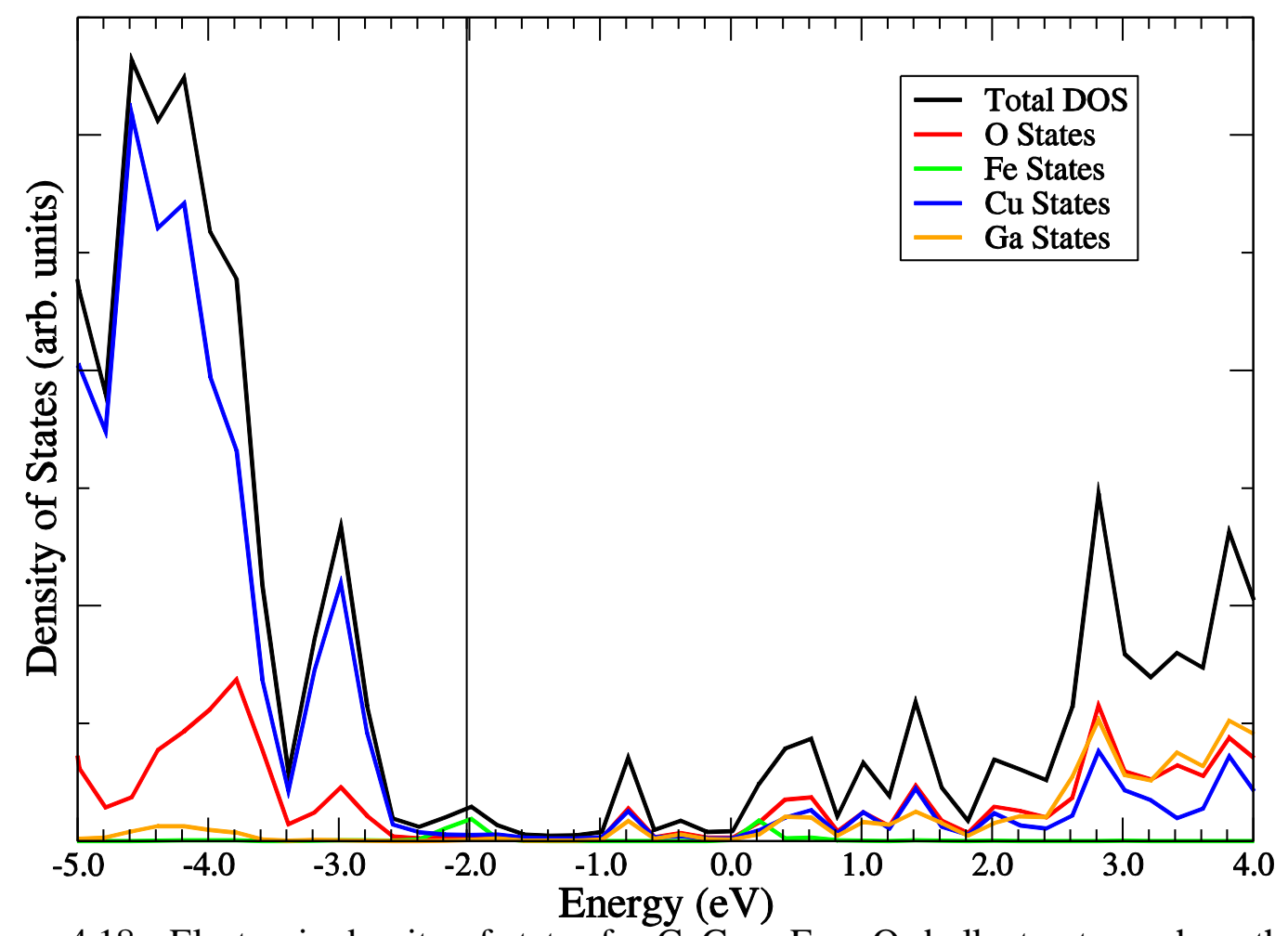

Figure 4.18. Electronic density of states for $\mathrm{CuGa}_{0.98} \mathrm{Fe}_{0.02} \mathrm{O}_{2}$ bulk structure where the Fermi level is denoted by the black vertical line.

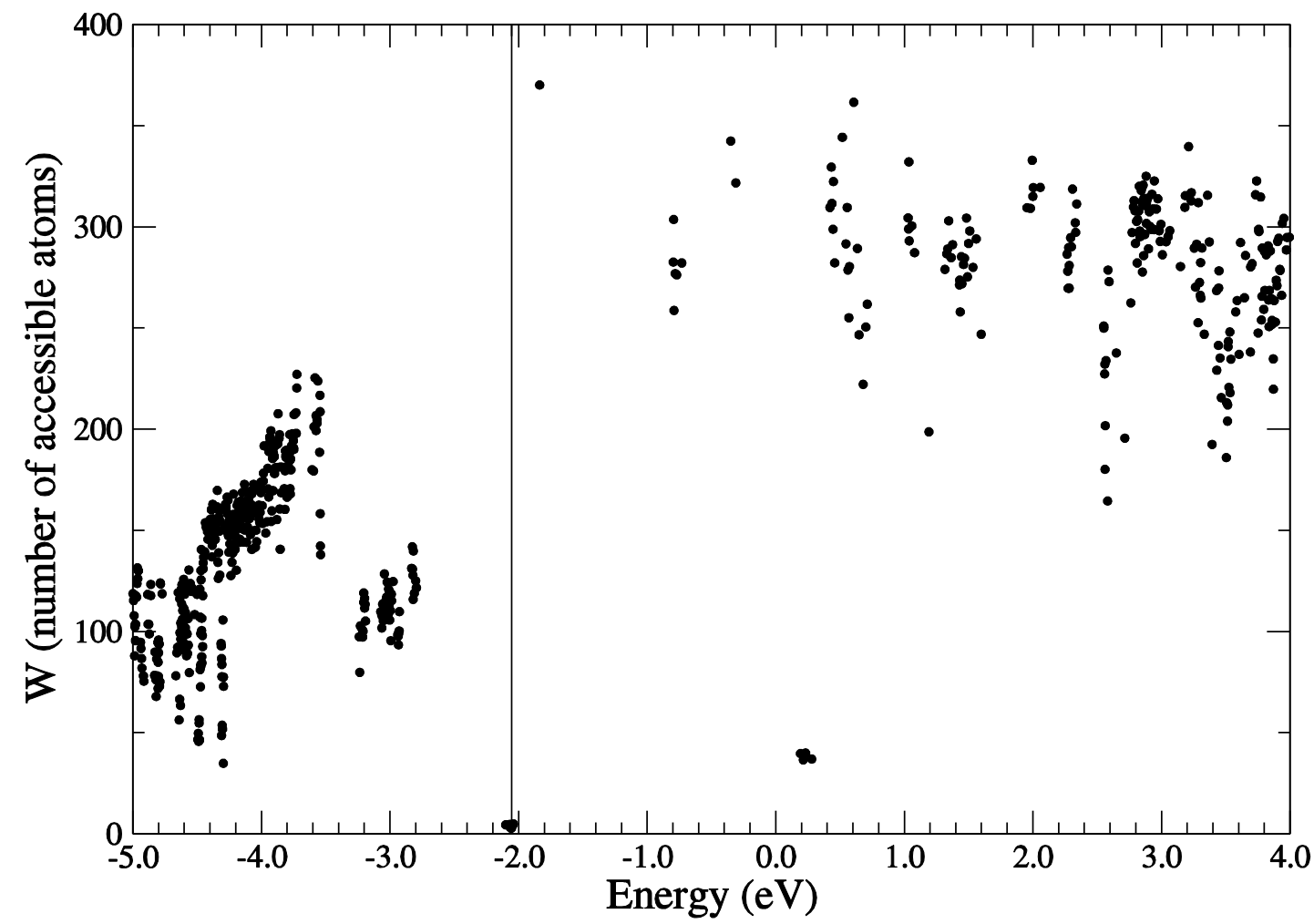

Figure 4.19. W plot for $\mathrm{CuGa}_{0.98} \mathrm{Fe}_{0.02} \mathrm{O}_{2}$ bulk structure where the valence band edge is denoted by the black vertical line. 


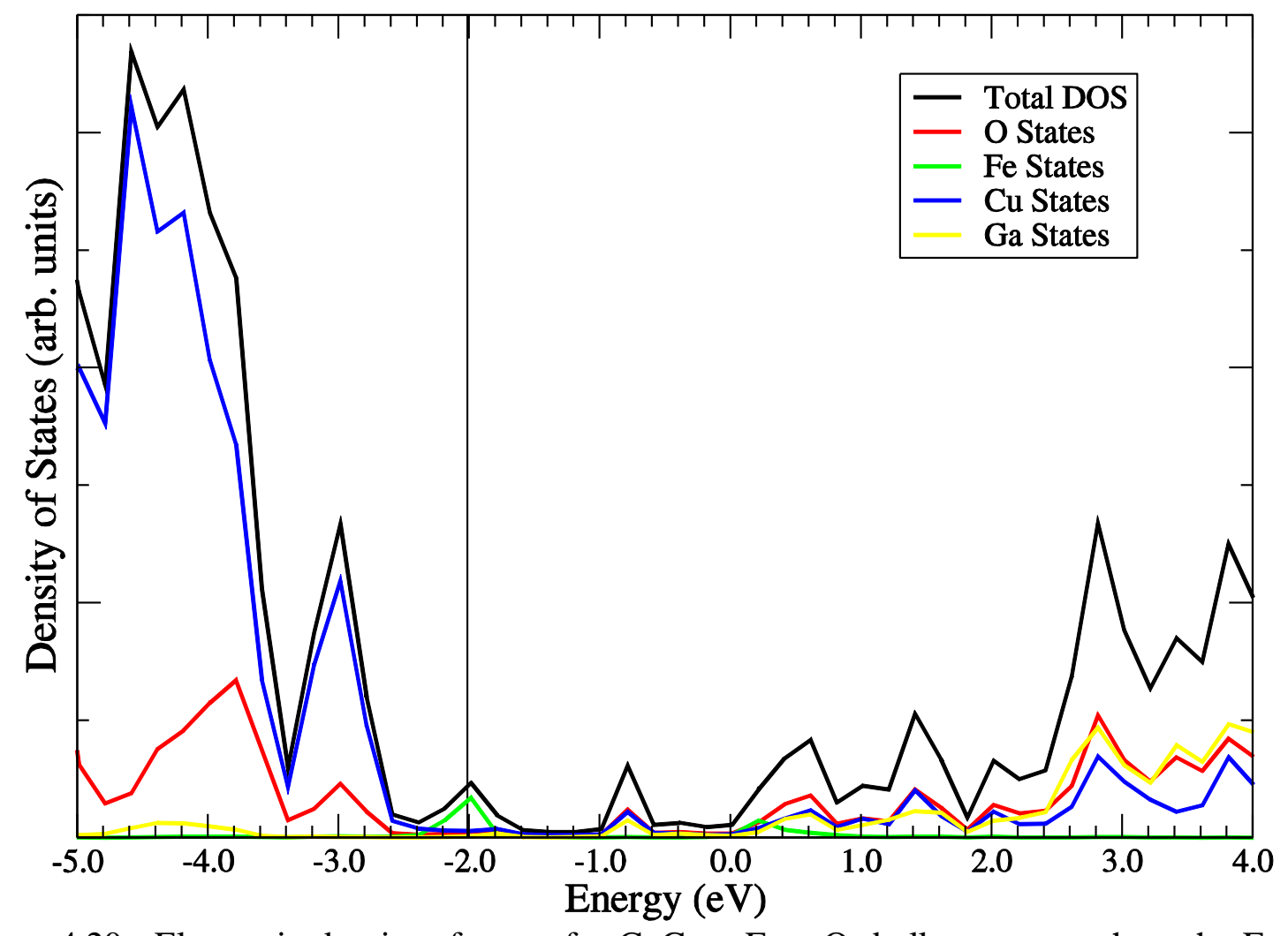

Figure 4.20. Electronic density of states for $\mathrm{CuGa}_{0.97} \mathrm{Fe}_{0.03} \mathrm{O}_{2}$ bulk structure where the Fermi level is denoted by the black vertical line.

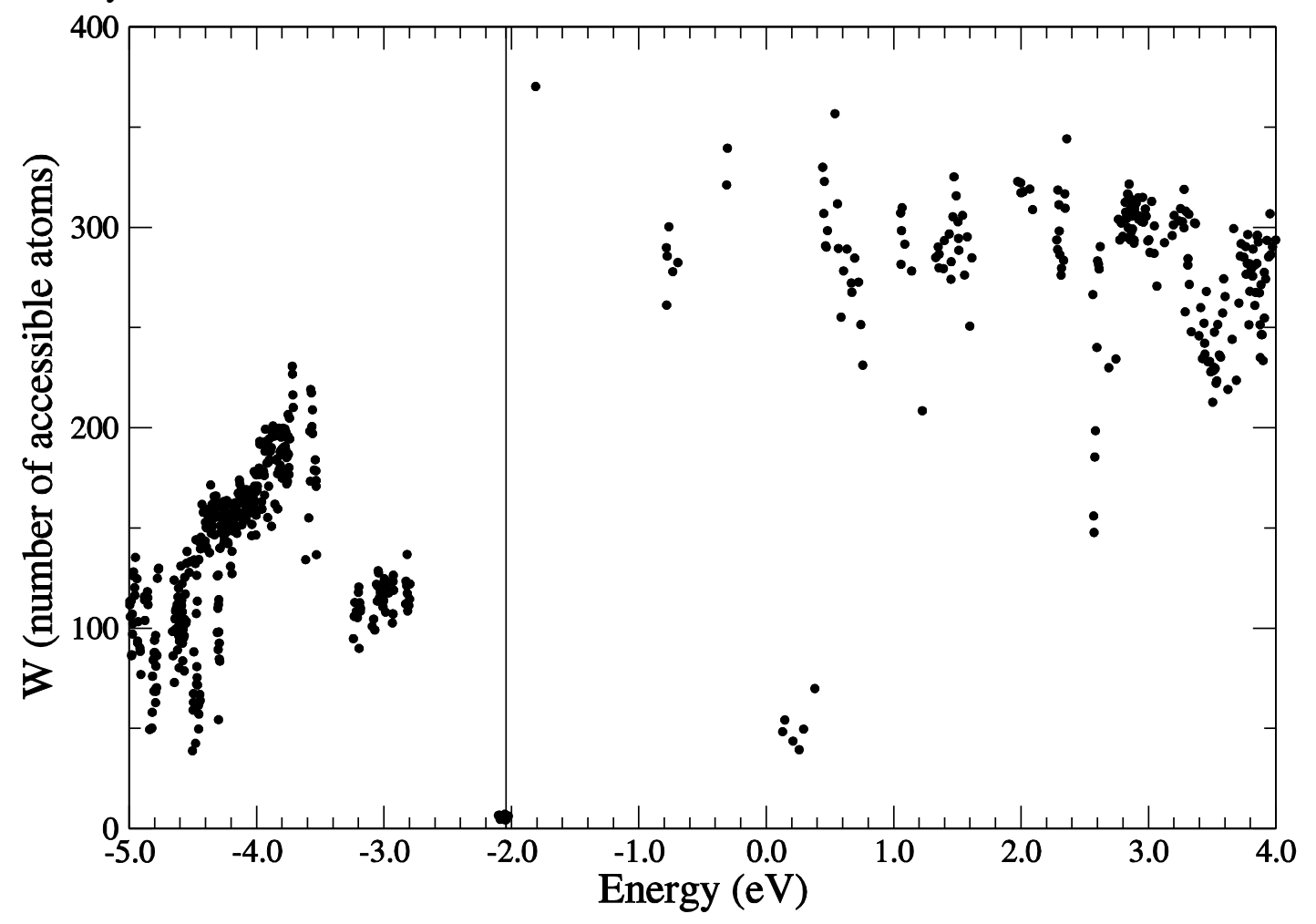

Figure 4.21. W plot for $\mathrm{CuGa}_{0.97} \mathrm{Fe}_{0.03} \mathrm{O}_{2}$ bulk structure where the valence band edge is denoted by the black vertical line. 


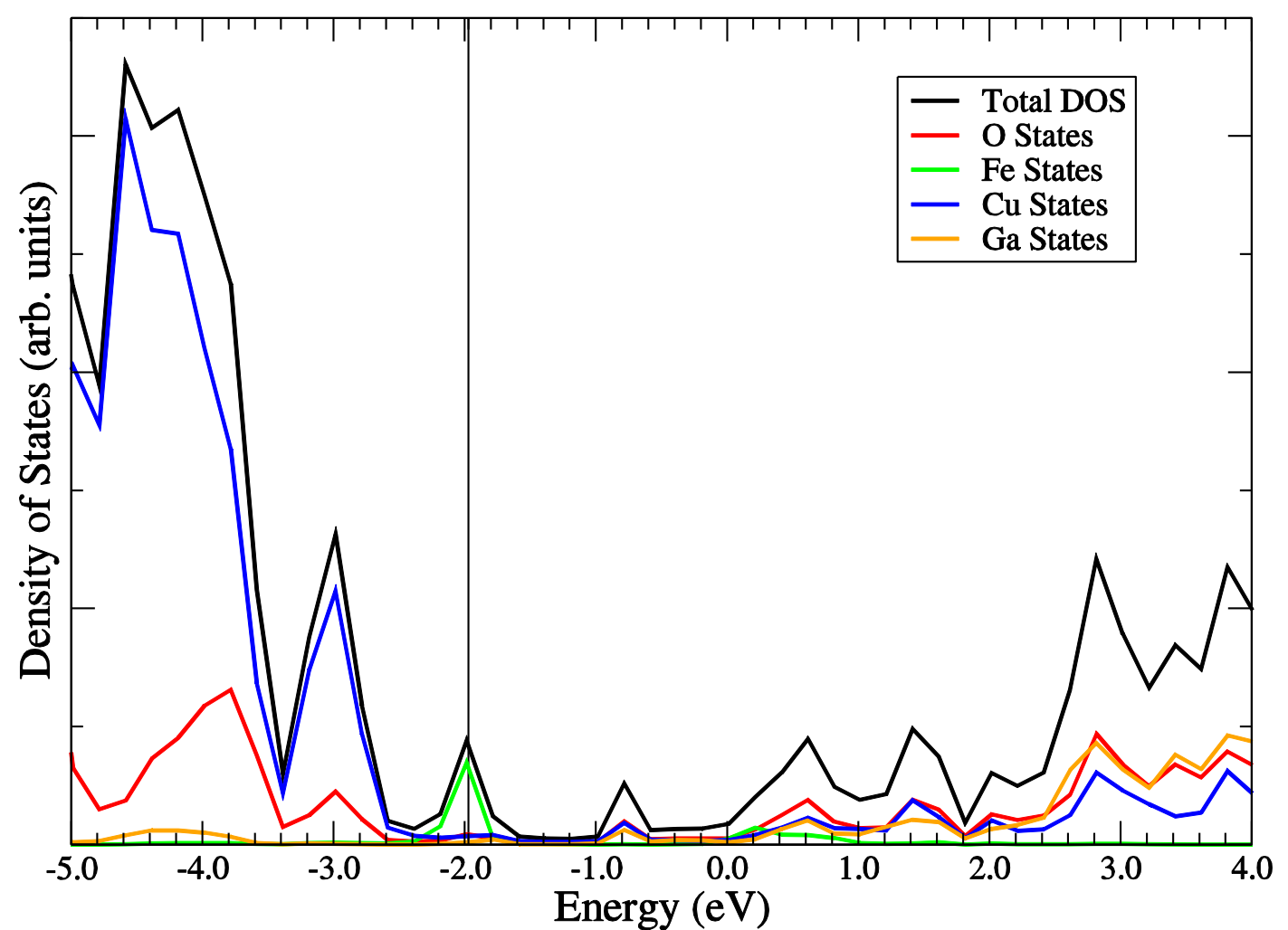

Figure 4.22. Electronic density of states for $\mathrm{CuGa}_{0.96} \mathrm{Fe}_{0.04} \mathrm{O}_{2}$ bulk structure where the Fermi level is denoted by the black vertical line.

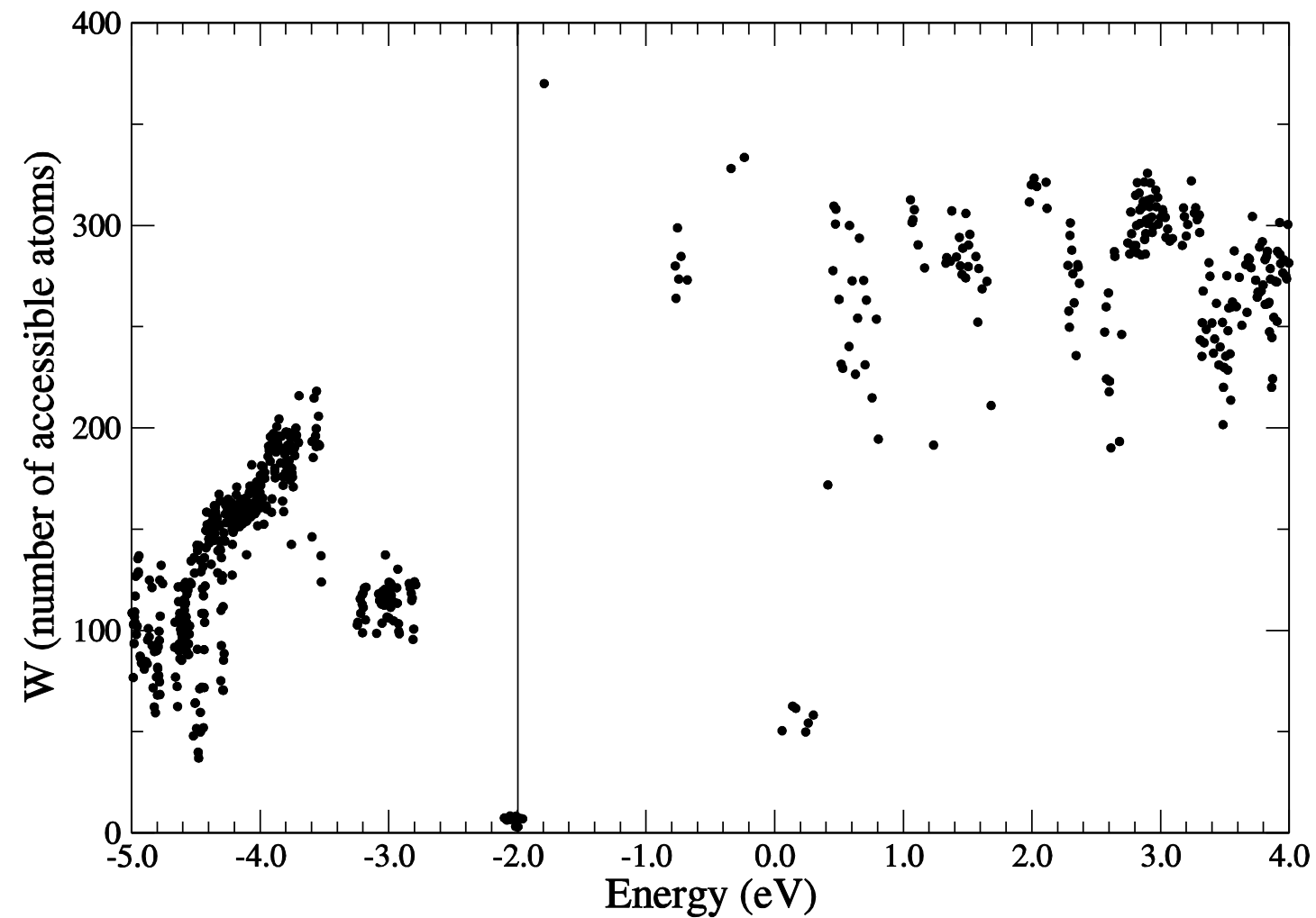

Figure 4.23. $\mathrm{W}$ plot for $\mathrm{CuGa}_{0.96} \mathrm{Fe}_{0.04} \mathrm{O}_{2}$ bulk structure where the valence band edge is denoted by the black vertical line. 


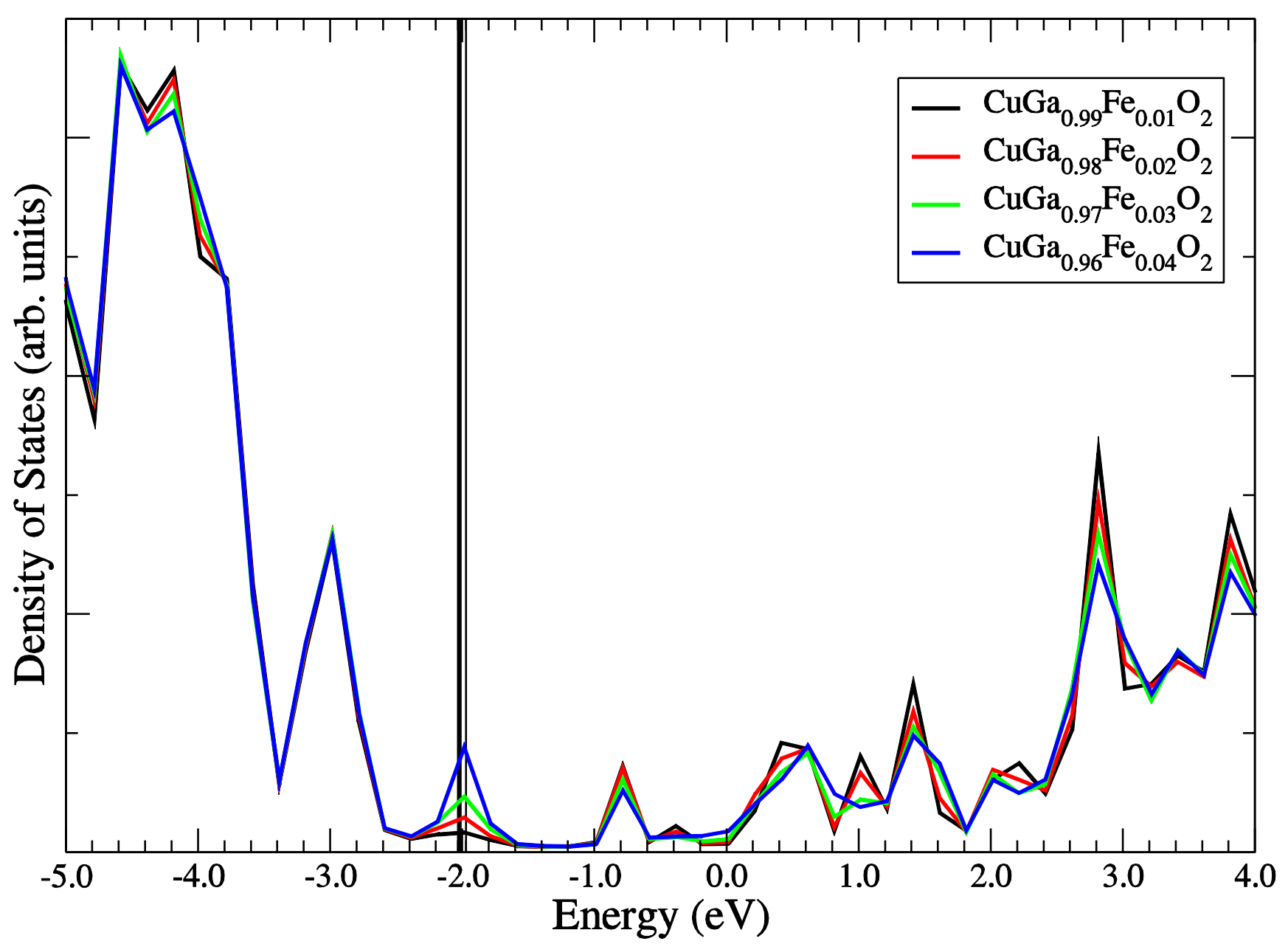

Figure 4.24. DOS plots for structures with doping percentages $0.01,0.02,0.03$, and 0.04 overlaid together with vertical lines denoting the Fermi levels increasing in energy with increasing doping percentage.

Even with these minimal doping percentages, broadening of the features between $0 \mathrm{eV}$ and $2 \mathrm{eV}$ occurs, though it becomes more definite as the percentage increases. The increasing delocalization of states at $0.0 \mathrm{eV}$ as percentage doping increases may be a measurement of strain on the system from symmetry breaking. Once these states become sufficiently delocalized, visible light absorption is maximized and these states are preferred. Also, the highly delocalized states at $-1.0 \mathrm{eV}$ appear to spread out as doping percentage increases. This spreading of states may also explain the condition for maximum enhancement of visible light absorption near 5 percent doping. 


\section{Discussion and Conclusions}

We find that B-site doping in $\mathrm{CuGaO}_{2}$ with $\mathrm{Fe}$ to obtain $\mathrm{CuGa}_{1-\mathrm{x}} \mathrm{Fe}_{\mathrm{x}} \mathrm{O}_{2}$ leads to the breaking of inversion symmetry and therefore lowers the band gap and increases visible light absorption. This process seems to find no added benefit at doping percentages above 5\%. Even so, these materials cannot use visible light to reduce $\mathrm{CO}_{2}$ to $\mathrm{CO}$, but still require $\mathrm{UV}$ light to so. This appears to be due to a realignment of the band edge potentials rather than the creation of recombination centers as the broadened states in the conduction band appear from the $\mathrm{W}$ plots to be highly delocalized. We conclude the enhancement in visible light absorption is due to broadened and accessible oxygen states in the conduction band. Because these states are not due to iron orbitals, this insinuates that the new states are caused by the strain added to the lattice due to the addition of iron atoms rather than acting as localized iron recombination centers.

These materials could be useful in the photoelectrochemical reduction of materials other than $\mathrm{CO}_{2}$, such as hydrogen production by water-splitting. It is also possible that there could be a different combination delafossite which is more suitable for the $\mathrm{CO}_{2}$ reduction reaction. Given at least 5 possible A-site materials and 13 possible B-site materials, naively there are 390 binary delafossite materials which could be explored. This would serve as a perfect computational highthroughput condensed matter problem, and would fit into the materials genome project quite well

${ }^{43}$. Going even further, another area to explore are ternary delafossite materials of the form $\mathrm{AB}^{1}{ }_{1 \text { - }}$ ${ }_{x-y} \mathrm{~B}^{2}{ }_{x} \mathrm{~B}_{y}^{3} \mathrm{O}_{2}$ to find a intermediate band gap material which behaves similarly to the recently discovered $\mathrm{V}: \mathrm{SnS}_{2}{ }^{44}$.

This research only looks at one possible doping configuration for each $\mathrm{CuGa}_{1-\mathrm{x}} \mathrm{Fe}_{\mathrm{x}} \mathrm{O}_{2}$, it would be helpful to perform multiple doping configurations of each structure to 
understand preferred patterns of doping as well as differences in the effectiveness of visible light enhancement of these patterns.

Through this research, tools have been created to quickly ready and perform high throughput calculations on multiple structures at each doping percentage. Due to this research, Bsite doping ab inito studies are also being started on $\mathrm{CuInO}_{2}, \mathrm{CuAlO}_{2}, \mathrm{NaInO}_{2}$ and $\mathrm{NaFeO}_{2}$ using these tools to accelerate progress. Although $\mathrm{CuGaO}_{2} \mathrm{~B}$-site doped with $\mathrm{Fe}$ did not prove to be an acceptable reducing catalyst of $\mathrm{CO}_{2}$ with visible light, nonetheless this research set the ground work for other candidate B-site doped delafossite materials to be explored for this and other purposes. 


\section{BIBLIOGRAPHY}

1.House, K. Z.; Harvey, C. F.; Aziz, M. J.; Schrag, D. P., The energy penalty of post-combustion $\mathrm{CO}_{2}$ capture \& storage and its implications for retrofitting the U.S. installed base. Energy \& Environmental Science 2009, 2 (2), 193-205.

2.(a) Haszeldine, R. S., Carbon Capture and Storage: How Green Can Black Be? Science 2009, 325 (5948), 1647-1652; (b) Pires, J. C. M.; Martins, F. G.; Alvim-Ferraz, M. C. M.; Simões, M., Recent developments on carbon capture and storage: An overview. Chemical Engineering Research and Design 2011, 89 (9), 1446-1460.

3.Benson, E. E.; Kubiak, C. P.; Sathrum, A. J.; Smieja, J. M., Electrocatalytic and homogeneous approaches to conversion of $\mathrm{CO}_{2}$ to liquid fuels. Chem Soc Rev 2009, 38 (1), 89-99.

4.(a) Adachi, K.; Ohta, K.; Mizuno, T., Photocatalytic reduction of carbon-dioxide to hydrocarbon using copper-loaded titanium-dioxide. Solar Energy 1994, 53 (2), 187-190; (b) Anpo, M.; Yamashita, H.; Ichihashi, Y.; Ehara, S., Photocatalytic reduction of $\mathrm{CO}_{2}$ with $\mathrm{H}_{2} \mathrm{O}$ on various titanium-oxide catalysts. Journal of Electroanalytical Chemistry 1995, 396 (1-2), 21-26; (c) Inoue, T.; Fujishima, A.; Konishi, S.; Honda, K., Photoelectrocatalytic reduction of carbon-dioxide in aqueous suspensions of semiconductor powders. Nature 1979, 277 (5698), 637-638; (d) Solymosi, F.; Tombacz, I., Photocatalytic reaction of $\mathrm{H}_{2} \mathrm{O}+\mathrm{CO}_{2}$ over pure and doped $\mathrm{Rh} / \mathrm{TiO}_{2}$. Catalysis Letters 1994, 27 (1-2), 61-65; (e) Tseng, I. H.; Chang, W. C.; Wu, J. C. S., Photoreduction of $\mathrm{CO}_{2}$ using sol-gel derived titania and titania-supported copper catalysts. Applied Catalysis BEnvironmental 2002, 37 (1), 37-48; (f) Varghese, O. K.; Paulose, M.; LaTempa, T. J.; Grimes, C. A., High-rate solar photocatalytic conversion of $\mathrm{CO}_{2}$ and water vapor to hydrocarbon fuels. Nano Letters 2009, 9 (2), 731-737; (g) Wang, C. J.; Thompson, R. L.; Baltrus, J.; Matranga, C., Visible Light Photoreduction of $\mathrm{CO}_{2}$ Using $\mathrm{CdSe} / \mathrm{Pt} / \mathrm{TiO}_{2}$ Heterostructured Catalysts. Journal of Physical Chemistry Letters 2010, 1 (1), 48-53; (h) Yamashita, H.; Kamada, N.; He, H.; Tanaka, K.; Ehara, S.; Anpo, M., Reduction of $\mathrm{CO} 2$ with $\mathrm{H} 2 \mathrm{O}$ on $\mathrm{TiO} 2(100)$ and $\mathrm{TiO} 2(110)$ single-crystals under UV-irradiation. Chemistry Letters 1994, (5), 855-858.

5.Marquardt, M. A.; Ashmore, N. A.; Cann, D. P., Crystal chemistry and electrical properties of the delafossite structure. Thin Solid Films 2006, 496 (1), 146-156.

6.Huda, M. N.; Yan, Y. F.; Walsh, A.; Wei, S. H.; Al-Jassim, M. M., Group-IIIA versus IIIB delafossites: Electronic structure study. Phys Rev B 2009, 80 (3).

7.(a) Younsi, M.; Saadi, S.; Bouguelia, A.; Aider, A.; Trari, M., Synthesis and characterization of oxygen-rich delafossite $\mathrm{CuYO}_{2+\mathrm{x}}$ Application to $\mathrm{H}_{2}$-photo production. Solar Energy Materials and Solar Cells 2007, 91 (12), 1102-1109; (b) Nie, X. L.; Wei, S. H.; Zhang, S. B., Bipolar doping and band-gap anomalies in delafossite transparent conductive oxides. Physical Review Letters 2002, 88 (6). 
8.(a) Benko, F. A.; Koffyberg, F. P., Opto-electronic properties of CuAlO2. Journal of Physics and Chemistry of Solids 1984, 45 (1), 57-59; (b) Benko, F. A.; Koffyberg, F. P., Optoelectronic properties of para-type and normal-type delafossite, $\mathrm{CuFeO}_{2}$. Journal of Physics and Chemistry of Solids 1987, 48 (5), 431-434; (c) Benko, F. A.; Koffyberg, F. P., The Optical Interband-Transitions of the Semiconductor $\mathrm{CuGaO}_{2}$. Physica Status Solidi a-Applied Research 1986, 94 (1), 231-234; (d) Kawazoe, H.; Yasukawa, M.; Hyodo, H.; Kurita, M.; Yanagi, H.; Hosono, H., P-type electrical conduction in transparent thin films of $\mathrm{CuAlO}_{2}$. Nature 1997, 389 (6654), 939-942; (e) Yanagi, H.; Hase, T.; Ibuki, S.; Ueda, K.; Hosono, H., Bipolarity in electrical conduction of transparent oxide semiconductor $\mathrm{CuInO}_{2}$ with delafossite structure. Applied Physics Letters 2001, 78 (11), 15831585; (f) Prewitt, C. T.; Shannon, R. D.; Rogers, D. B., Chemistry of Noble Metal Oxides .2. Crystal Structures of $\mathrm{PtCoO}_{2}, \mathrm{PdCoO}_{2}, \mathrm{CuFeO}_{2}$, and $\mathrm{AgFeO}_{2}$. Inorganic Chemistry 1971, 10 (4), 719-\&; (g) Rogers, D. B.; Shannon, R. D.; Prewitt, C. T.; Gillson, J. L., Chemistry of noble metal oxides .3. Electrical transport properties and crystal chemistry of $\mathrm{ABO}_{2}$ compounds with delafossite structure. Inorganic Chemistry 1971, 10 (4), 723-\&; (h) Shannon, R. D.; Rogers, D. B.; Prewitt, C. T., Chemistry of noble metal oxides .1. Synthesis and properties of $\mathrm{ABO}_{2}$ delafossite compounds. Inorganic Chemistry 1971, 10 (4), 713-\&.

9.Huda, M. N.; Yan, Y. F.; Walsh, A.; Wei, S. H.; Al-Jassim, M. M., Symmetry-breaking-induced enhancement of visible light absorption in delafossite alloys. Applied Physics Letters 2009, 94 (25).

10.Oppenheimer., M. B. a. R., Zur Quantentheorie der Molekeln. Ann. Phys. (Leipzig) 1927, 84 (20).

11.(a) Haycock, B. Calculation on electronic and optical properties of nanoscale systems. Dublin Institute of Technology, 2011, Thesis; (b) Leach, A. R., Molecular modelling : principles and applications. 2nd ed.; Prentice Hall: Harlow, England ; New York, 2001; p xxiii, 744 p., [16] p. of plates.

12.Kohn, W.; Sham, L. J., Quantum Density Oscillations in an Inhomogeneous Electron Gas. Phys Rev 1965, 137 (6A), 1697-\&.

13.Kresse, G.; Furthmuller, J., Efficiency of ab-initio total energy calculations for metals and semiconductors using a plane-wave basis set. Comp Mater Sci 1996, 6 (1), 15-50.

14.Gonze, X.; Amadon, B.; Anglade, P. M.; Beuken, J. M.; Bottin, F.; Boulanger, P.; Bruneval, F.; Caliste, D.; Caracas, R.; Cote, M.; Deutsch, T.; Genovese, L.; Ghosez, P.; Giantomassi, M.; Goedecker, S.; Hamann, D. R.; Hermet, P.; Jollet, F.; Jomard, G.; Leroux, S.; Mancini, M.; Mazevet, S.; Oliveira, M. J. T.; Onida, G.; Pouillon, Y.; Rangel, T.; Rignanese, G. M.; Sangalli, D.; Shaltaf, R.; Torrent, M.; Verstraete, M. J.; Zerah, G.; Zwanziger, J. W., ABINIT: Firstprinciples approach to material and nanosystem properties. Computer Physics Communications 2009, 180 (12), 2582-2615. 
15.(a) Demkov, A. A.; Ortega, J.; Sankey, O. F.; Grumbach, M. P., Electronic-structure approach for complex silicas. Phys Rev B 1995, 52 (3), 1618-1630; (b) Lewis, J. P.; Jelinek, P.; Ortega, J.; Demkov, A. A.; Trabada, D. G.; Haycock, B.; Wang, H.; Adams, G.; Tomfohr, J. K.; Abad, E.; Wang, H.; Drabold, D. A., Advances and applications in the FIREBALL ab initio tight-binding molecular-dynamics formalism. Physica Status Solidi B-Basic Solid State Physics 2011, 248 (9), 1989-2007.

16.Soler, J. M.; Artacho, E.; Gale, J. D.; Garcia, A.; Junquera, J.; Ordejon, P.; Sanchez-Portal, D., The SIESTA method for ab initio order-N materials simulation. Journal of Physics-Condensed Matter 2002, 14 (11), 2745-2779.

17.Sankey, O. F.; Niklewski, D. J., AbInitio multicenter tight-binding model for moleculardynamics simulations and other applications in covalent systems. Phys Rev B 1989, 40 (6), 39793995.

18.Plimpton, S., Fast Parallel Algorithms for Short-Range Molecular-Dynamics. J Comput Phys 1995, $117(1), 1-19$.

19.Basanta, M. A.; Dappe, Y. J.; Jelínek, P.; Ortega, J., Optimized atomic-like orbitals for firstprinciples tight-binding molecular dynamics. Comp Mater Sci 2007, 39 (4), 759-766.

20.Finnis, M. W., The harris functional applied to surface and vacancy formation energies in aluminum. Journal of Physics-Condensed Matter 1990, 2 (2), 331-342.

21.(a) Matthew, W.; Foulkes, C.; Haydock, R., Tight-binding models and density-functional theory. Phys Rev B 1989, 39 (17), 12520-12536; (b) Harris, J., Simplified method for calculating the energy of weakly interacting fragments. Phys Rev B 1985, 31 (4), 1770-1779.

22.Sankey, O. F.; Demkov, A. A.; Windl, W.; Fritsch, J. H.; Lewis, J. P.; Fuentes-Cabrera, M., The application of approximate density functionals to complex systems. International Journal of Quantum Chemistry 1998, 69 (3), 327-340.

23.Henderson, B.; Imbusch, G. F., Optical spectroscopy of inorganic solids. Clarendon Press ;Oxford University Press: Oxford [Oxfordshire]New York, 1989; p xvi, 645 p.

24.(a) Kohler, B. U.; Jansen, M., Synthesis and structural data of delafossites $\mathrm{CuAlO}_{2}, \mathrm{CuGaO}_{2}$, $\mathrm{CuScO}_{2}, \mathrm{CuYO}_{2}$. Zeitschrift Fur Anorganische Und Allgemeine Chemie 1986, 543 (12), 73-80; (b) Soller, W.; Thompson, A. J., Physical Review 1935, 47, 644.

25.Shannon, R. D.; Prewitt, C. T., Effective ionic radii in oxides and fluorides. Acta Crystallographica Section B-Structural Crystallography and Crystal Chemistry 1969, B 25, 925$\&$.

26.Denton, A. R.; Ashcroft, N. W., Vegard Law. Physical Review A 1991, 43 (6), 3161-3164. 
27.Würfel, P.; Würfel, U., Physics of solar cells : from basic principles to advanced concepts. 2nd, updated and expanded ed.; Wiley-VCH: Weinheim, 2009; p xii, 244 p.

28.(a) Blakemore, J. S., Semiconductor statistics. Pergamon Press: Oxford, New York,, 1962; p 381 p; (b) Goudon, T.; Miljanovic, V.; Schmeiser, C., On the Shockley-Read-Hall Model: Generation-recombination in semiconductors. Siam Journal on Applied Mathematics 2007, 67 (4), 1183-1201.

29.Gratzel, M., Photoelectrochemical cells. Nature 2001, 414 (6861), 338-344.

30.Dean, J. A.; Lange, N. A., Lange's handbook of chemistry. 14th ed.; McGraw-Hill: New York, 1992; p 1 v. (various pagings).

31.Setyawan, W.; Curtarolo, S., High-throughput electronic band structure calculations: Challenges and tools. Comp Mater Sci 2010, 49 (2), 299-312.

32.Monkhorst, H. J.; Pack, J. D., Special Points for Brillouin-Zone Integrations. Phys Rev B 1976, 13 (12), 5188-5192.

33.Momma, K.; Izumi, F., VESTA: a three-dimensional visualization system for electronic and structural analysis. Journal of Applied Crystallography 2008, 41, 653-658.

34.Lekse, J. W.; Underwood, M. K.; Lewis, J. P.; Matranga, C., Synthesis, Characterization, Electronic Structure, and Photocatalytic Behavior of $\mathrm{CuGaO}_{2}$ and $\mathrm{CuGa}_{1-\mathrm{x}} \mathrm{Fe}_{\mathrm{x}} \mathrm{O}_{2}(\mathrm{x}=0.05,0.10$, 0.15, 0.20) Delafossites. Journal of Physical Chemistry C 2012, 116 (2), 1865-1872.

35.Ibach, H.; Lüth, H., Solid-state physics : an introduction to principles of materials science. 4th extensively updated and enl. ed.; Springer: Berlin ; New York, 2009; p xiv, 533 p.

36.Lennard-Jones, J. E., The Electronic Structure of Some Diatomic Molecules Trans. Faraday Soc. 1929, 25.

37.Ashcroft, N. W.; Mermin, N. D., Solid state physics. Holt: New York,, 1976; p xxi, 826 p.

38.J. P. Lewis, T. E. C., III, H. Wang, E. Starikow, and O. F. Sankey., Dynamically amorphous character of electronic states in poly(dA)-poly(dT) DNA. J. Phys. Chem. B 2003, 107, 2581-2587.

39.Hamann, D. R., Generalized Norm-Conserving Pseudopotentials. Phys Rev B 1989, 40 (5), 2980-2987.

40.Troullier, N.; Martins, J. L., Efficient Pseudopotentials for Plane-Wave Calculations II. Operators for Fast Iterative Diagonalization. Phys Rev B 1991, 43 (11), 8861-8869.

41.(a) Jelinek, P.; Wang, H.; Lewis, J. P.; Sankey, O. F.; Ortega, J., Multicenter approach to the exchange-correlation interactions in ab initio tight-binding methods. Phys Rev B 2005, 71 (23), 
235101; (b) Tafen, D. N.; Wang, J.; Wu, N.; Lewis, J. P., Visible light photocatalytic activity in nitrogen-doped $\mathrm{TiO}_{2}$ nanobelts. AIP: 2009; Vol. 94, p 093101; (c) Wang, C. J.; Thompson, R. L.; Ohodnicki, P.; Baltrus, J.; Matranga, C., Size-dependent photocatalytic reduction of CO2 with $\mathrm{PbS}$ quantum dot sensitized $\mathrm{TiO} 2$ heterostructured photocatalysts. Journal of Materials Chemistry 2011, Unpublished; (d) Wang, H.; Lewis, J. P., Effects of dopant states on photoactivity in carbondoped TiO2. Journal of Physics-Condensed Matter 2005, 17 (21), L209-L213; (e) Wang, H.; Lewis, J. P., Second-generation photocatalytic materials: anion-doped TiO2. Journal of PhysicsCondensed Matter 2006, 18 (2), 421-434; (f) Wang, H.; Lewis, J. P., Localization of Frontier Orbitals on Anatase Nanoparticles Impacts Water Adsorption. The Journal of Physical Chemistry C 2009, 113 (38), 16631-16637; (g) Wang, J.; Tafen, D. N.; Lewis, J. P.; Hong, Z.; Manivannan, A.; Zhi, M.; Li, M.; Wu, N., Origin of Photocatalytic Activity of Nitrogen-Doped $\mathrm{TiO}_{2} \mathrm{Nanobelts.}$ Journal of the American Chemical Society 2009, 131 (34), 12290-12297; (h) Wu, N.; Wang, J.; Tafen, D. N.; Wang, H.; Zheng, J.-G.; Lewis, J. P.; Liu, X.; Leonard, S. S.; Manivannan, A., Shape-Enhanced Photocatalytic Activity of Single-Crystalline Anatase TiO2 (101) Nanobelts. Journal of the American Chemical Society 2010, 132 (19), 6679-6685.

42.(a) Omeiri, S.; Bellal, B.; Bouguelia, A.; Bessekhouad, Y.; Trari, M., Electrochemical and photoelectrochemical characterization of $\mathrm{CuFeO}_{2}$ single crystal. Journal of Solid State Electrochemistry 2009, 13 (9), 1395-1401; (b) Buljan, A.; Alemany, P.; Ruiz, E., Electronic structure and bonding in $\mathrm{CuMO}_{2}(\mathrm{M}=\mathrm{Al}, \mathrm{Ga}, \mathrm{Y})$ delafossite-type oxides: An ab initio study. Journal of Physical Chemistry B 1999, 103 (38), 8060-8066.

43.Jain, A.; Hautier, G.; Moore, C. J.; Ong, S. P.; Fischer, C. C.; Mueller, T.; Persson, K. A.; Ceder, G., A high-throughput infrastructure for density functional theory calculations. Comp Mater Sci 2011, 50 (8), 2295-2310.

44.Wahnon, P.; Conesa, J. C.; Palacios, P.; Lucena, R.; Aguilera, I.; Seminovski, Y.; Fresno, F., Vdoped SnS(2): a new intermediate band material for a better use of the solar spectrum. Physical Chemistry Chemical Physics 2011, 13 (45), 20401-20407. 